\title{
A Dual Sensor for Biogenic Amines and Oxygen Based on Genipin Immobilized in Edible Calcium Alginate Gel Beads
}

Ian Mallov, Fiona Jeeva, Chris Caputo

Submitted date: 06/05/2020 - Posted date: 07/05/2020

Licence: CC BY-NC-ND 4.0

Citation information: Mallov, Ian; Jeeva, Fiona; Caputo, Chris (2020): A Dual Sensor for Biogenic Amines and Oxygen Based on Genipin Immobilized in Edible Calcium Alginate Gel Beads. ChemRxiv. Preprint. https://doi.org/10.26434/chemrxiv.12252323.v1

Food is often wasted due to real or perceived concerns about preservation and shelf life. Thus, precise, accurate and consumer-friendly methods of indicating whether food is safe for consumers are drawing great interest. The colorimetric sensing of biogenic amines released as food degrades is a potential way of determining the quality of the food. Herein, we report the use of genipin, a naturally occurring iridoid, as a dual colorimetric sensor for both oxygen and biogenic amines. Immobilization of genipin in edible calcium alginate beads demonstrates that it is a capable sensor for amine vapors and can be immobilized in a non-toxic, food-friendly matrix.

File list (3)

Manuscript Submission.pdf (913.79 KiB) view on ChemRxiv • download file view on ChemRxiv - download file 


\title{
A Dual Sensor for Biogenic Amines and Oxygen Based on Genipin Immobilized in Edible Calcium Alginate Gel Beads
}

\author{
Ian Mallov, Fiona Jeeva and Christopher B. Caputo* \\ Department of Chemistry, York University, Toronto, ON, M3J 1P3, Canada
}

\begin{abstract}
Food is often wasted due to real or perceived concerns about preservation and shelf life. Thus, precise, accurate and consumer-friendly methods of indicating whether food is safe for consumers are drawing great interest. The colorimetric sensing of biogenic amines released as food degrades is a potential way of determining the quality of the food. Herein, we report the use of genipin, a naturally occurring iridoid, as a dual colorimetric sensor for both oxygen and biogenic amines. Immobilization of genipin in edible calcium alginate beads demonstrates that it is a capable sensor for amine vapors and can be immobilized in a non-toxic, food-friendly matrix.
\end{abstract}

\section{Introduction}

Biogenic amines (BAs) are a major indicator of food spoilage, particularly in meat and fish, but also in such food and drink as beer, red wine, mushrooms, and aged cheese. ${ }^{1}$ BAs are produced when food is exposed to oxygen gas, allowing for microbial oxidative hydrodecarboxylation of amino acids. They are responsible for the distinctive odor of rotting meat, although adverse effects to human health can occur in concentrations lower than the odor threshold. ${ }^{2-4}$ Globally, nearly $1 / 3$ of all food is wasted, thus precise, accurate and consumer-friendly methods of indicating whether food is safe to consumers are drawing great interest. ${ }^{5}$

A number of instrumental methods and techniques to detect BA's have been developed which utilize conventional chromatography methods, ${ }^{2}$ chemoresistivity, ${ }^{4,6}$ or biological sensing. ${ }^{7}$ However, these methods have limitations for practical use in detecting food spoilage, as the analyses require large or specialized equipment, and these impede their application by the public. Progress has been made on this front and an accessible electrical sensor was recently reported utilizing printed electronic gas sensors onto cellulose fibers to detect ammonia. ${ }^{8}$ On the other hand, visual detection sensors have garnered attention as reliable systems which do not require significant instrumentation. Visual sensors can be subdivided into fluorescent or colorimetric sensors. Both benefit from enhanced sensitivity, ease of use and real-time analysis. However, colorimetric sensors have the advantage of being detectable by the naked eye. Considerable research has been undertaken to develop colorimetric sensors for BAs. Some recent highlights of BA detection include copper-containing complexes which have shown selective detection for spermidine and spermine, ${ }^{9}$ light-activated diarylethene probes, ${ }^{10}$ aza-bodipy derivatives, ${ }^{11}$ and derivatives of Meldrum's activated furan ${ }^{12,13}$ (Scheme 1 ). 
Tang, 2016
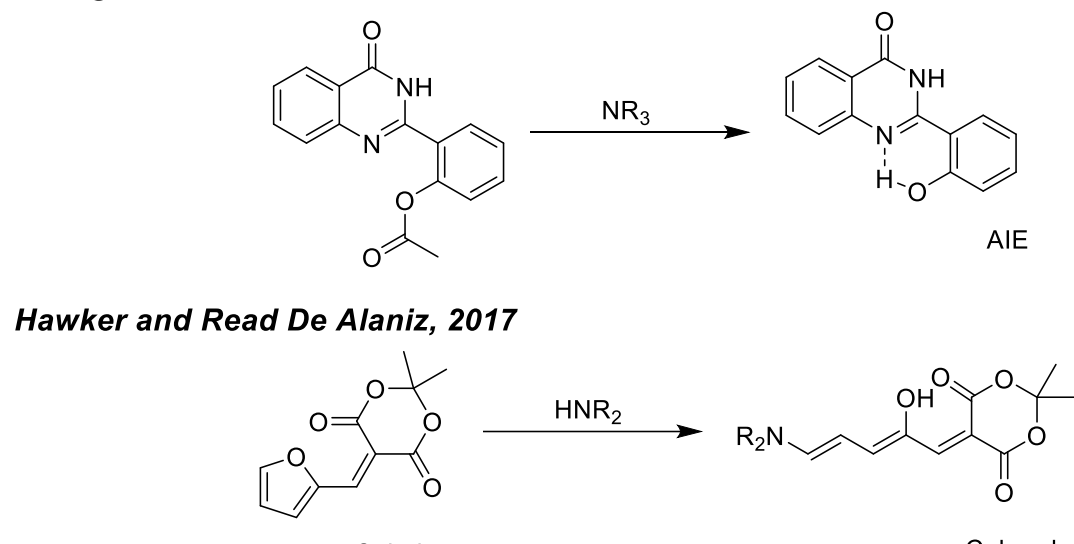

This Work

Colorless

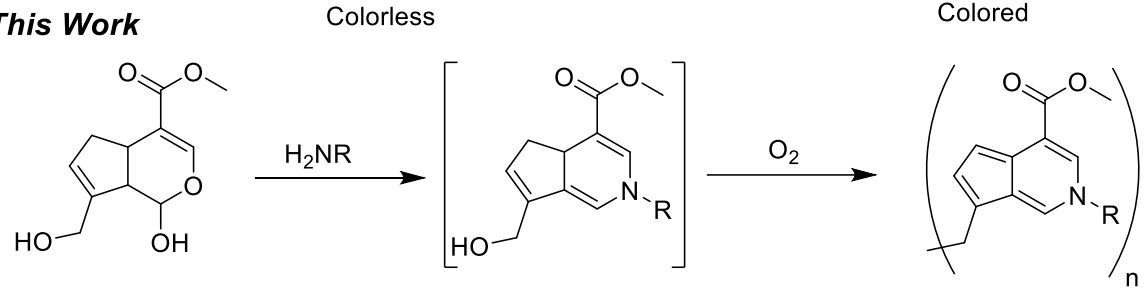

Red Colored

Blue Conjugated Oligomeric Products

Scheme 1 - Recent Examples of Small Molecules for BA Sensing

Previously reported colorimetric BA sensors still have several drawbacks. Many are non-specific for amine substitution (BAs are generally primary amines) or are made of materials which would be incompatible with food (i.e. toxic or non-edible materials). Recent studies have addressed the latter drawback by utilizing the $\mathrm{pH}$ sensitivity of anthocyanins found in cabbage extracts to detect BA. ${ }^{14,15}$ However, these sensors are not specific for primary BA. Most significantly, current colorimetric sensors cannot detect the cause of food spoilage, oxygen gas, so false positives could occur through simple detection of amino acids found in the food. To address this issue, modified atmospheric packaging is gaining in popularity as a way of excluding oxygen from food and extending shelf lives. ${ }^{16}$

An ideal colorimetric sensor for real-time food spoilage would sense gaseous BAs at low concentrations and rapidly undergo a distinct color change at a reasonable reaction rate. Further, this sensor must have the ability to selectively detect the primary amines as well as the source of the degradation, oxygen gas. This ideal sensor would be composed of cheap, low-toxicity materials derived from edible sources and possess a minimal environmental footprint. We aimed to design a sensor satisfying as many of these criteria as possible.

Genipin, (Scheme 1, bottom) a naturally occurring, colorless iridoid, has found numerous applications in diverse fields, most commonly used as a non-toxic cross-linking reagent for biomaterials. ${ }^{17,18}$ As an edible fruit extract, genipin also has relatively low toxicity; a recent study shows that neither genipin nor the resultant gardenia blue dye exhibit genotoxicity. ${ }^{19}$ This transformation from a colorless iridoid to dye can be attributed to the fact that genipin reacts with amines through a double condensation reaction forming an oxidatively unstable 
dihydropyridine intermediate (Scheme 1, bottom). In the presence of oxygen, this intermediate further undergoes oxidative oligomerization/polymerization resulting in a brilliant blue dye. ${ }^{20}$ However, to the best of our knowledge genipin has yet to find applications as a sensor, despite this unique reactivity positioning it as an ideal candidate for a colorimetric sensor for food degradation.

We sought to study the ability of genipin to react with BAs and confirm the selectivity towards a broad range of amines. Herein, we describe the use of genipin as the first dual colorimetric sensor for both $\mathrm{O}_{2}$ and BAs in solution and provide a proof of concept in an immobilized form.

\section{Experimental Section}

Materials. All chemical reagents or solvents were purchased from Fisher Scientific or Millipore Sigma and used as received unless otherwise indicated. Genipin was kindly donated by Inkbox Ink and purity verified independently by ${ }^{1} \mathrm{H}$ NMR analysis. Food-grade sodium alginate was purchased from BYOB Cocktail Emporium in Toronto and used without purification. NMR spectra were obtained at $25{ }^{\circ} \mathrm{C}$ on either a Bruker $700 \mathrm{MHz}$ Spectrometer, Bruker DRX $600 \mathrm{MHz}$ Spectrometer, Bruker ARX $400 \mathrm{MHz}$ Spectrometer or, Bruker ARX $300 \mathrm{MHz}$ Spectrometer. Chemical shifts are reported relative to $\mathrm{SiMe}_{4}$ and referenced to the residual solvent signal $\left({ }^{1} \mathrm{H}\right.$, $\left.{ }^{13} \mathrm{C}\left\{{ }^{1} \mathrm{H}\right\}\right)$. NMR spectra were analyzed using either TopSpin 4.0.1 or MestReNova 6.0.2-5475 software. Chemical shifts are reported in ppm and coupling constants as scalar values in $\mathrm{Hz}$. The conventional abbreviations were used as follows: $s$ (singlet), $d$ (doublet), $t$ (triplet), q (quartet), dd (doublet of doublets), $m$ (multiplet), br (broad). Absorption measurements were recorded with a Cary 5000 UV-vis-NIR Spectrophotometer from Agilent Technologies. Recordings were obtained at $25^{\circ} \mathrm{C}$ and taken with the instrument operating in dual beam mode and referenced to methanol. All absorption experiments were conducted in quartz cuvettes $(1 \mathrm{~cm} \times 1 \mathrm{~cm})$ equipped with a Teflon seal.

UV-visible absorbance detection of biogenic amines in solution $22.6 \mathrm{mg}(0.10 \mathrm{mmol})$ of genipin was dissolved in $45.0 \mathrm{~mL}$ methanol in a single-neck round-bottom flask by stirring for five minutes with a magnetic stirrer. For $1: 1$ solutions of genipin:BA, $0.10 \mathrm{mmol}$ of BA were added to a separate pre-weighed vial and rinsed into the genipin solution with a further $5.0 \mathrm{~mL}$ methanol to prepare $50.0 \mathrm{~mL}$ of solution of concentrations $2.0 \times 10^{-3} \mathrm{M}$ in genipin and $\mathrm{BA}$. For solutions lower in concentration of $\mathrm{BA}$, stock solutions $0.020 \mathrm{M}$ in $\mathrm{BA}$ were prepared in methanol. Appropriate volumes of additional methanol were added as needed to dilute to desired concentrations, with total volumes made up to $50.0 \mathrm{~mL}$. Round-bottomed flasks containing solutions were left open to allow exposure to oxygen and stirred for 48-72 hours. Measurement of UV-visible absorbances were carried out by decanting solutions into a graduated cylinder at time of measurement, making up the total volume to $50.0 \mathrm{~mL}$ with methanol to compensate for evaporation, decanting back into the round-bottom flask and stirring for 5 minutes to achieve homogeneity. Samples were then transferred into a quartz cuvette for analysis. Single-neck (1" diameter) round-bottom flasks were chosen as reaction vessels after verifying that rates of evaporation of stirred 
methanol at room temperature ensured that solution volumes never fell below $40 \mathrm{~mL}$ during the course of the reaction.

Preparation of genipin-embedded calcium alginate gel beads. 1, 10, 50, or $100 \mathrm{mg}$ of genipin was dissolved in $10.0 \mathrm{~mL}$ of distilled water in a $100 \mathrm{~mL}$ beaker. In the case of the addition of 100 $\mathrm{mg}$ of genipin, heating to $80{ }^{\circ} \mathrm{C}$ was required for full dissolution. $200 \mathrm{mg}$ of sodium alginate powder was added with vigorous stirring to form the sodium alginate gel. Using a variation of the simple dripping (extrusion) technique described by Bhandari et. al., ${ }^{21}$ a $1 \mathrm{~mL}$ disposable syringe without a needle was employed to uptake $1.00 \mathrm{~mL}$ of sodium alginate gel. Due to dripping of gel from the outside of the syringe, the first 1-2 drops were dispensed back into the gel mixture. The remainder was dispensed dropwise from a height of $10 \mathrm{~cm}$ above the surface of $25.0 \mathrm{~mL}$ of a 1.0 $\% \mathrm{w} / \mathrm{v} \mathrm{CaCl} 2$ solution contained in a $125 \mathrm{~mL}$ Erlenmeyer flask. 12-15 beads were dispensed in this manner until the syringe contained $>0.1 \mathrm{~mL}$ of gel, at which point the syringe was re-filled to avoid size decrease of gel beads due to air bubbles inside the syringe, and lack of material. After 100-110 beads had been dispensed in this manner, using $75-80 \%$ of the sodium alginate gel mass, the remaining $\mathrm{CaCl}_{2}$ solution was decanted from the beads and the beads were washed with 10 $\mathrm{mL}$ of an aqueous solution of $0.1 \mathrm{M} \mathrm{CH}{ }_{3} \mathrm{COOH} / \mathrm{CH}_{3} \mathrm{COONa} \mathrm{pH} 4$ buffer. Beads were then dispensed onto a watch glass, separated using tweezers and permitted to stand 1 hour to allow excess water to run onto the glass. The beads were then removed from the watch glass and stored in a sealed vial in a refrigerator at $4{ }^{\circ} \mathrm{C}$.

General Procedure for reaction of genipin immobilized in calcium alginate gel beads with primary amine vapors. 1-10 genipin-embedded calcium alginate gel beads were placed in a 20 $\mathrm{mL}$ vial using a clean pair of tweezers. $0.10 \mathrm{mmol}$ of primary amine was added to a 1-dram vial (or Eppendorf tube) which was placed, uncapped, inside the 4-dram vial containing the beads, and the larger vial was sealed with a cap and either left at room temperature or placed in a refrigerator at $4{ }^{\circ} \mathrm{C}$ and monitored visually for up to 48 hours days.

In the case where ten gel beads were exposed to $0.10 \mathrm{mmol}$ amine, after 24 hours at room temperature the gel beads remained colourless, while after 48 hours all ten beads had turned blue. In the case where only one bead was used instead of ten beads, the single bead turned deep blue within 24 hours.

To achieve dissolution of gel beads to obtain UV-vis spectra after specified reaction times of 6-48 hours, 10 dyed genipin-containing gel beads were added to $3.5 \mathrm{~mL}$ of a $55 \mathrm{mM}$ solution of sodium citrate to allow dissolution of gel beads by cation exchange mediated by the citrate cation chelator. ${ }^{22}$ Although some of the blue dye became insoluble particulate in this aqueous solution, precluding qualitative UV-vis absorption measurements, enough remained in solution to qualitatively observe the blue dye by UV-vis spectroscopy.

Reaction of genipin immobilized in calcium alginate gel beads with raw chicken. 10 genipinembedded calcium alginate gel beads produced no more than seven days previously were placed in each of two $20 \mathrm{~mL}$ vials using a clean pair of tweezers. A piece of chicken breast purchased 
within the previous 24 hours from a commercial grocery store was cut into slices of mass 5-6 grams and one slice was placed in each vial using a separate, clean pair of tweezers, such that the gel beads were separated from the surface of the raw chicken. The vials were sealed with a plastic screw cap. One was left at room temperature, while one was refrigerated at $4{ }^{\circ} \mathrm{C}$. Both samples were monitored visually and photographed at hours 1, 2, 3 and 6.

\section{Results and Discussion}

To confirm the selectivity of genipin for primary amines, the reactions with a series of amines in methanol solutions were monitored over periods of 48-72 hours at room temperature. These reactions were performed at concentrations of $2.0 \times 10^{-3} \mathrm{M}$ for both genipin and amine, in open vessels to permit diffusion of $\mathrm{O}_{2}$ into the system to promote blue dye formation. Benzylamine, $\mathrm{N}$-methylbenzylamine, and $\mathrm{N}, \mathrm{N}$-dimethylbenzylamine were initially tested to represent a series of simple, analogous primary, secondary and tertiary amines, and reactions were monitored by UV-vis absorbance spectroscopy. Consistent with reported literature, formation of blue dye was only observed in the reaction of genipin with the primary benzylamine, with strong visible absorbance exhibited between 550-650 nm (Figure S4). Though solutions of tertiary and secondary amines, $\mathrm{N}, \mathrm{N}$-dimethylbenzylamine and $\mathrm{N}$-methylbenzylamine, did exhibit a colour change to reddish golden, they did not form the blue dye even after extended reaction times. Monitoring the reactions of $\mathrm{N}, \mathrm{N}$-dimethylbenzylamine and $\mathrm{N}$-methylbenzylamine, by ${ }^{1} \mathrm{H}$ NMR spectroscopy with genipin at greater concentrations does indicate a reaction between genipin and amine but it produced an intractable mixture of products. These studies unequivocally show the selectivity of genipin to react with primary amines to form a blue product.

Reactions with biogenic amines putrescine, cadaverine, tyramine and histamine also exhibited formation of deep blue dye (Figure 1). In these cases, an incubation period of 12-24 hours was required before strong absorbances with maxima at 590-610 $\mathrm{nm}$ were observed. The incubation time is attributable to the time required for growth of conjugated oligomers absorbing in the red region. Although reactions were monitored for up to 120 hours, in all cases, $\lambda_{\max }$ and molar attenuation coefficient values demonstrated only minimal change after 48 hours (e.g. Figure S5). Interestingly, molar attenuation coefficients varied widely between reactions of genipin with different $\mathrm{BA}$, ranging from $1.2 \times 10^{2} \mathrm{Lmol}^{-1} \mathrm{~cm}^{-1}$ in putrescine to $4.4 \times 10^{3} \mathrm{Lmol}^{-1} \mathrm{~cm}^{-1}$ in tyramine. It is not obvious from differences in molecular structure of the amines why significant variance is observed in molar attenuation coefficients of the solutions of dyes. 


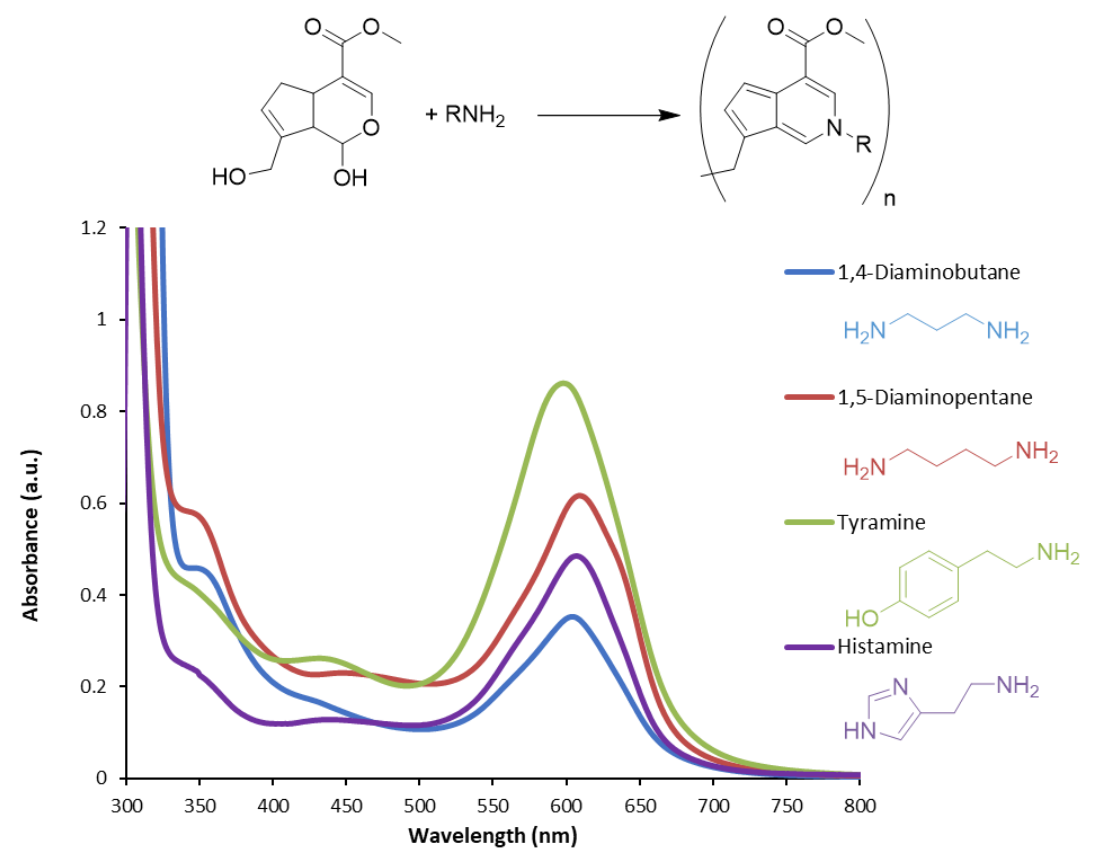

Figure 1 - Stacked UV-vis Spectra for the reaction of genipin with BAs. Dilution factors - 1,4diaminobutane (no dilution); 1,5-diaminopentane (5x dilution); Tyramine (10x dilution); Histamine (20x dilution).

Unsurprisingly, when reactions were carried out at increased concentrations of genipin and amine, the blue dye formed much more rapidly, for example within 4 hours at concentrations of $0.20 \mathrm{M}$ (Figure S10)).

From these solution studies, the limit of visual detection of amines with a $0.50 \mathrm{M}$ solution of genipin in 50:50 methanol:water was found to vary depending on BA used. Putrescine and tyramine, the tested amines whose dyes exhibited the lowest and highest molar attenuation coefficients, respectively, were examined. Solutions of genipin in methanol exhibit a dull golden tinge, while presence of the blue dyes were confirmed visually by a change in colour to dull green, the result of the presence of both blue dye and the golden hue from remaining unreacted genipin. Tyramine could be visually detected at concentrations of $6.9 \mathrm{ppm}$ after 24 hours (Figure S27), while the detection limit for putrescine was nearly an order of magnitude higher at $44 \mathrm{ppm}$ after 24 hours. This trend correlates with the measured trend in molar attenuation coefficient measured for the dyes incorporating putrescine or tyramine units (converted from molarity).

Enticingly, the reaction of putrescine with genipin in the absence of oxygen produced a different response (Figure 2). Using degassed water and an atmosphere of nitrogen, the solutions produced a golden red coloration with no indication of the blue response as indicated by the $\lambda_{\max }$ at $\sim 600 \mathrm{~nm}$. Upon exposure to air after 48 hours, the solutions rapidly turned blue. To explore the sensitivity of the genipin and amine intermediate to oxygen gas, we found even $0.5 \%$ (5000 ppm) of oxygen gas (converted from moles of $\mathrm{H}_{2} \mathrm{O}_{2}$, see $\mathrm{SI}$ ) added under an atmosphere of $\mathrm{N}_{2}$ were enough to trigger the oxidative oligomerization reaction turning the sample blue over a 
period of 24 hours. This sensitivity is below the oxygen threshold established in modified atmospheric packaging ( 2\%) and in the desired range of sensitivity $\left(0.5-2 \% \mathrm{O}_{2}\right.$ level). ${ }^{16}$

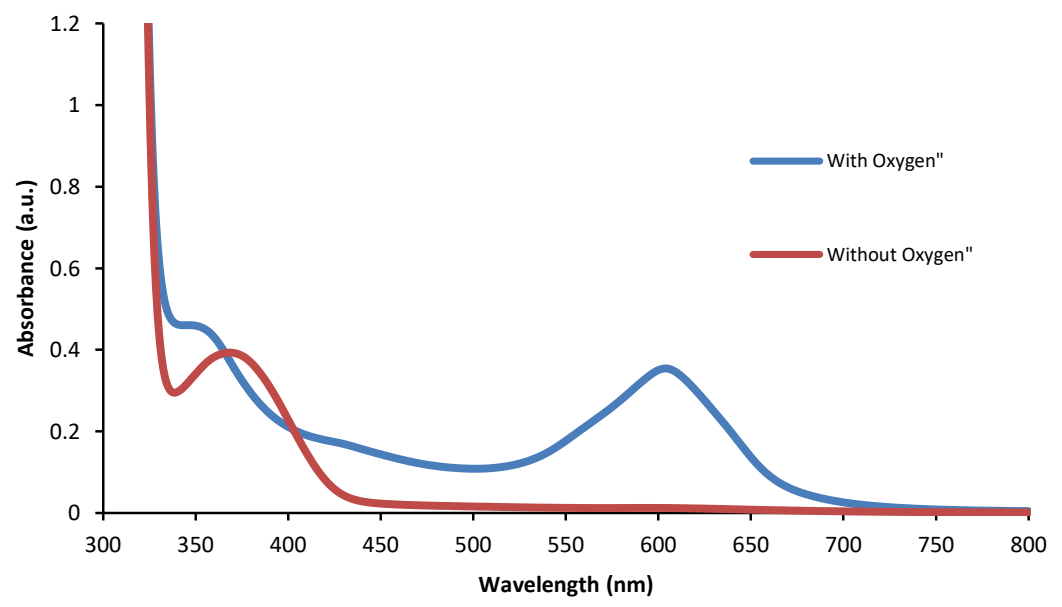

Figure 2 - UV-vis absorbance spectra of $2.0 \mathrm{mM}$ solutions of genipin in methanol in reaction with $2.0 \mathrm{mM}$ of 1,4-diaminobutane in the presence (blue) and absence (red) of $\mathrm{O}_{2}$ after 48 hours.

To demonstrate the applicability of our sensing platform for food spoilage detection, an immobilization method for genipin was developed using a calcium alginate gel matrix. Alginate gels are common edible food thickening agents derived from alginic acid sourced from seaweed or brown algae. ${ }^{22}$ Calcium alginate gel beads are easily produced from sodium alginate and permit penetration of small molecules through their pores. ${ }^{21}$ Genipin was embedded in these beads through modifying a literature procedure for the impregnation of calcium alginate gel beads with enzymes. ${ }^{21}$ After extensive screening, it was found that a concentration of $5 \mathrm{mg} / \mathrm{mL}$ of genipin in water, approximately equivalent to the room-temperature solubility of genipin in water, resulted in the most responsive beads. As a proof of concept, we selected the most volatile biogenic amine, putrescine, to test the sensing capabilities of the beads. Immobilized genipincontaining beads were exposed to the gaseous BA by adding $0.1 \mathrm{mmol}$ of putrescine to an open 1-dram vial and sealing the 1-dram vial inside a 4-dram vial containing 10 genipin-infused beads. The beads responded as expected and a blue colour developed upon exposure to BA vapors within 24 hours (Figure 3). To mimic conditions for food storage, these experiments were also undertaken at $4{ }^{\circ} \mathrm{C}$, and encouragingly the beads did not exhibit any color change, even after 48 hours (Figure S28). Gel beads could subsequently be dissolved in an aqueous citric acid solution for UV-vis absorbance analysis (Figure 3), although undissolved particulate matter interfered with quantitative measurements.

In analyzing the absorbance of solutions obtained from dissolving gel beads exposed for 48 hours to vials containing varying amounts of putrescine (Figure S29), it is evident that increasing amounts of amine result in increased intensity of the blue dye. The vapor pressure of linear putrescine has been reported as $259.3 \mathrm{~Pa}$ at $300.7 \mathrm{~K} .{ }^{23}$ Using the reported vapor pressure at 300.7 
$\mathrm{K}$ as an approximation for the vapor pressure at room temperature, it can be calculated from the ideal gas law that for the $18 \mathrm{~mL}$ closed volume of the 4-dram vial, evaporation of $<0.2 \mathrm{mg}$ of putrescine is required to reach equilibrium vapor pressure. Upon dissolution after reaction, solutions of gel beads exposed to $0.3 \mathrm{mg}, 1.2 \mathrm{mg}$ and $12 \mathrm{mg}$ of putrescine in open one-dram vials demonstrated significantly increased absorbance as the amount of amine was increased (Figure S29). Thus, it can be hypothesized that the intensity of the absorbance is more correctly correlated to the absolute amount of amine available for reaction, and the identity of that amine, than the vapor pressure.
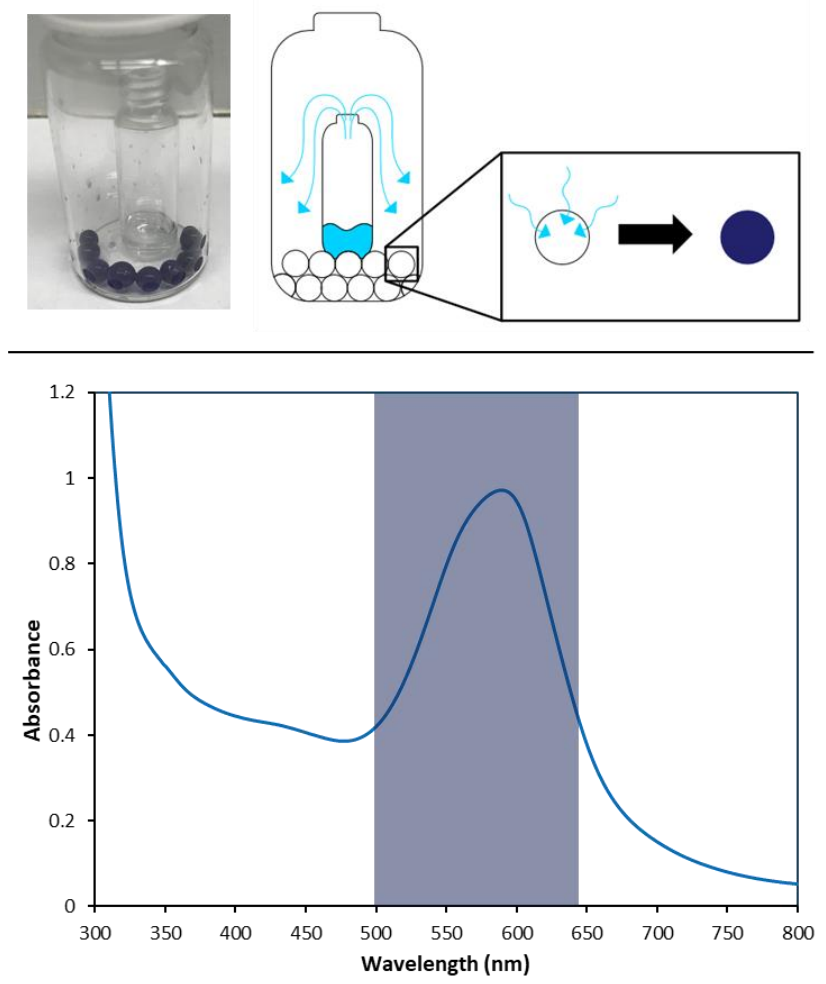

Figure 3: Genipin-impregnated beads after 24 hours exposure to putrescine vapours at room temperature

Having established the sensor's response to gaseous amines, we sought to test them against real food samples. Samples of chicken breast (5-6 grams each) were freshly prepared and stored in sealed vials with the genipin-impregnated beads. With the beads in close proximity to the meat, the sensors behaved as expected and turned blue upon exposure. Samples were stored both at room temperature and in a refrigerator at $4{ }^{\circ} \mathrm{C}$ to mimic typical storage conditions. To quantify the color difference between the beads, $\Delta E^{*}$ values were determined following commission internationale de l'éclairage (CIE) guidelines (Figure 4). A $\Delta E^{*}$ value $>5$ is considered to be distinguishable by the naked eye. ${ }^{12}$ This approach has previously been used to determine the effectiveness of small molecule amine sensors. ${ }^{12}$ The sensors were monitored over the course of 6 hours with images taken at time points of $1,2,3$ and 6 hours (Table S1). $\Delta E^{*}$ values of 38 and 0 
were obtained after 6 hours for the samples at room temperature and at $4{ }^{\circ} \mathrm{C}$, respectively. These values correspond to a significant blue coloration of the beads at room temperature versus no visible change in the refrigerator (Figure 4). The rate of colour appearance in these indicator beads is consistent with the USDA health advisory on raw poultry, which warns against leaving raw poultry at room temperature for longer than two hours. ${ }^{24}$

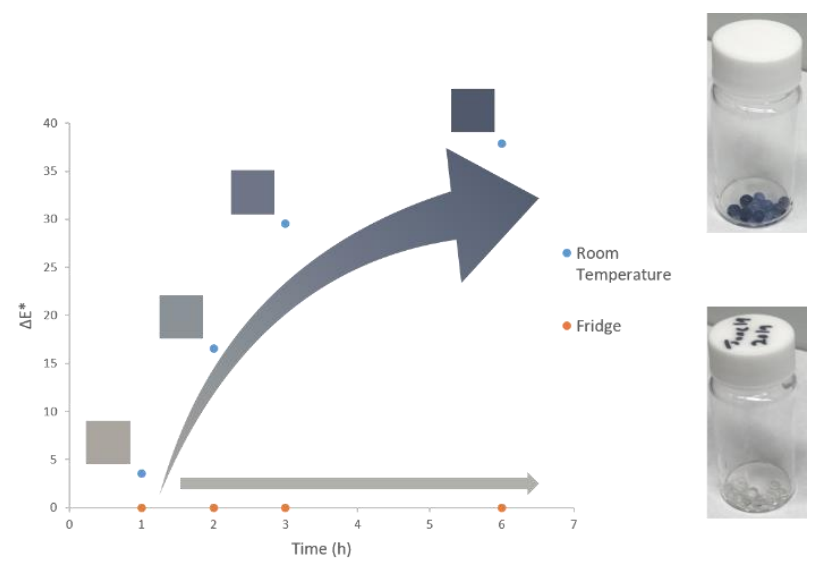

Figure 4: Change in color of Genipin Impregnated Beads in the presence of chicken, room temperature (blue) and $4{ }^{\circ} \mathrm{C}$ (orange).

\section{Conclusion}

In summary, this proof-of-principal study indicates that genipin can act as a highly selective dual sensor for both biogenic amines and $\mathrm{O}_{2}$. This uniquely positions genipin as an ideal colorimetric sensor for food spoilage as it can detect the root cause of this process through off-gases. Moreover, by immobilizing the sensor in an edible calcium alginate matrix, biogenic amine vapors were detected at very low concentrations. This was extended to monitoring chicken spoilage by the naked eye, indicating its potential in food spoilage detection.

\section{Acknowledgement}

The authors gratefully acknowledge financial support from NSERC of Canada, the Canadian Foundation for Innovation and Inkbox Ink. CBC is grateful for the award of a Canada Research Chair. The authors thank Professor Thomas Baumgartner for the use of the UV-vis spectrophotometer and valuable feedback.

\section{References}

1. Santos MHS. Biogenic amines: their importance in foods. Int J Food Microbiol. 29:213-31 (1996);

2. Önal A. A review: Current analytical methods for the determination of biogenic amines in foods. Food Chem. 103:1475-86 (2007);

3. Naila A, Flint S, Fletcher G, Bremer P, Meerdink G. Control of Biogenic Amines in FoodExisting and Emerging Approaches. J Food Sci. 75:R139-50 (2010); 
4. Liu SF, Petty AR, Sazama GT, Swager TM. Single-Walled Carbon

Nanotube/Metalloporphyrin Composites for the Chemiresistive Detection of Amines and Meat Spoilage. Angew. Chem. Int Ed. 54:6554-7 (2015);

5. Aschemann-Witzel J. Waste not, want not, emit less: Reducing food waste in the supply chain and at home can help to reduce carbon emissions. Vol. 352, Science. 352:408-9 (2016);

6. Sotzing GA, Phend JN, Grubbs RH, Lewis NS. Highly Sensitive Detection and Discrimination of Biogenic Amines Utilizing Arrays of Polyaniline/Carbon Black Composite Vapor Detectors. Chem Mater. 12:593-5 (2000);

7. Yousefi H, Ali MM, Su H-M, Filipe CDM, Didar TF. Sentinel Wraps: Real-Time Monitoring of Food Contamination by Printing DNAzyme Probes on Food Packaging. ACS Nano. 12:3287-94 (2018);

8. Barandun G, Soprani M, Naficy S, Grell M, Kasimatis M, Chiu KL, Ponzoni A, Güder F. Cellulose Fibers Enable Near-Zero-Cost Electrical Sensing of Water-Soluble Gases. ACS Sensors. 4:1662-9 (2019);

9. Chopra S, Singh A, Venugopalan P, Singh N, Kaur N. Organic nanoparticles for visual detection of spermidine and spermine in vapors and aqueous phase. ACS Sustain Chem Eng. 5:1287-96 (2017)

10. Valderrey V, Bonasera A, Fredrich S, Hecht S. Light-Activated Sensitive Probes for Amine Detection. Angew. Chem. Int Ed. 56:1914-8 (2017);

11. Li L, Li W, Ran X, Wang L, Tang H, Cao D. A highly efficient, colorimetric and fluorescent probe for recognition of aliphatic primary amines based on a unique cascade chromophore reaction. Chem Commun. 55:9789-92 (2019);

12. Diaz YJ, Page ZA, Knight AS, Treat NJ, Hemmer JR, Hawker CJ, Read De Alaniz J. A Versatile and Highly Selective Colorimetric Sensor for the Detection of Amines. Chem. Eur J. 23:3562-6 (2017);

13. Chen Q, Diaz YJ, Hawker MC, Martinez MR, Page ZA, Xiao-An Zhang S, Hawker CJ, Read de Alaniz J. Stable Activated Furan and Donor-Acceptor Stenhouse Adduct Polymer Conjugates as Chemical and Thermal Sensors. Macromolecules. 52:4370-5 (2019);

14. Dudnyk I, Janeček E-R, Vaucher-Joset J, Stellacci F. Edible sensors for meat and seafood freshness. Sensors Actuators B Chem. 259:1108-12 (2018);

15. Huang X, Zou X, Shi J, Guo Y, Zhao J, Zhang J, Hao L. Determination of pork spoilage by colorimetric gas sensor array based on natural pigments. Food Chem. 145:549-54 (2014);

16. Mills A. Oxygen indicators and intelligent inks for packaging food. Chem. Soc. Rev. 34:1003-11 (2005).

17. Yan LP, Wang YJ, Ren L, Wu G, Caridade SG, Fan JB, Wang LY, Ji PH, Oliveira JM, Oliveira JT, Mano JF, Reis RL. Genipin-cross-linked collagen/chitosan biomimetic scaffolds for 
articular cartilage tissue engineering applications. J Biomed Mater Res Part A. 95A:46575 (2010);

18. Butler MF, Ng Y-F, Pudney PDA. Mechanism and kinetics of the crosslinking reaction between biopolymers containing primary amine groups and genipin. J Polym Sci Part $A$ Polym Chem. 41:3941-53 (2003);

19. Tsai C-C, Huang R-N, Sung H-W, Liang HC. In vitro evaluation of the genotoxicity of a naturally occurring crosslinking agent (genipin) for biologic tissue fixation. J Biomed Mater Res. 52:58-65 (2000);

20. Touyama R, Takeda Y, Inoue K, Kawamura Ik, Yatsuzuka M, Ikumoto T, Shingu T, Yokoi T, Inouye H. Studies on the Blue Pigments Produced from Genipin and Methylamine. I. Structures of the Brownish-Red Pigments, Intermediates Leading to the Blue Pigments. Chem Pharm Bull. 42:668-73 (1994);

21. Ching $\mathrm{SH}$, Bansal N, Bhandari B. Alginate gel particles-A review of production techniques and physical properties. Crit Rev Food Sci Nutr. 57:1133-52 (2017);

22. Goh $\mathrm{CH}$, Heng PWS, Chan LW. Alginates as a useful natural polymer for microencapsulation and therapeutic applications. Vol. 88, Carbohydrate Polymers. p. 1122012.

23. Pozdeev VA, Verevkin SP. Vapor pressure and enthalpy of vaporization of linear aliphatic alkanediamines. J Chem Thermodyn. 43:1791-9 (2011);

24. https://www.fsis.usda.gov/wps/portal/fsis/topics/food-safety-education/getanswers/food-safety-fact-sheets/safe-food-handling/keep-food-safe-food-safetybasics/ct_index 


\section{Supporting Information}

A Dual Sensor for Biogenic Amines and Oxygen Based on Genipin Immobilized in Edible Calcium Alginate Gel Beads

Ian Mallov, Fiona Jeeva and Christopher B. Caputo*

Department of Chemistry, York University, Toronto, ON, M3J 1P3, Canada 


\section{Table of Contents}

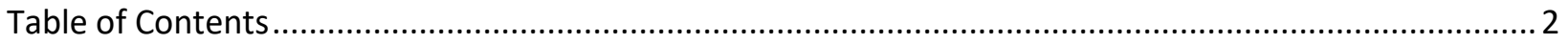

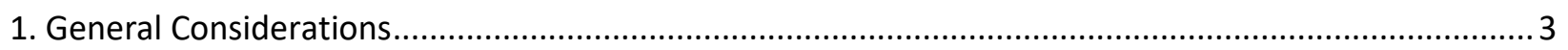

2. UV-Visible Absorbance Spectra of Starting Materials................................................................. 4

3. UV-Vis Absorbance Spectra - Reaction of Amines with Genipin in Solution.......................................... 7

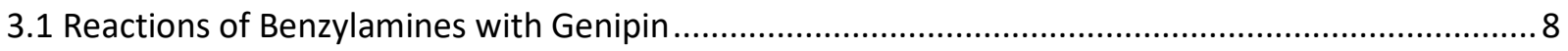

3.2 Reactions of Genipin with Putrescine (1,4-Diaminobutane) …................................................... 9

3.3 Reaction of Genipin with Putrescine With Addition of Chemically-Generated Oxygen.................. 13

3.4 Reactions of Genipin with Cadaverine (1,5-Diaminopentane) ..................................................... 20

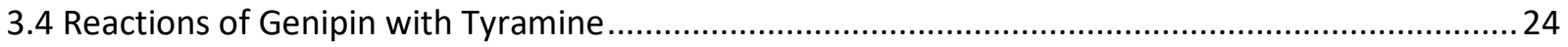

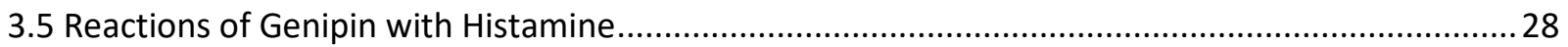

3.6 Estimating the Visual Detection Limit of Amines with a 0.500 M Solution of Genipin ................... 32

4. Colorimetric Response of Genipin-Infused Gel Beads Exposed to Amine Vapor .................................35

4.1 Genipin-Infused Gel Beads Exposed to Putrescine Vapor............................................................ 35

5. Colorimetric Response of Genipin-Infused Gel Beads Exposed to Raw Chicken ..................................39

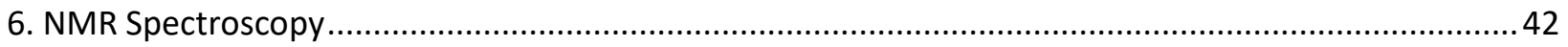




\section{General Considerations}

All chemical reagents or solvents were purchased from Fisher Scientific or Millipore Sigma and used as received unless otherwise indicated. Genipin was kindly donated by Inkbox Ink and purity verified independently by ${ }^{1} \mathrm{H}$ NMR analysis. Food-grade sodium alginate was purchased from BYOB Cocktail Emporium in Toronto and used without purification. NMR spectra were obtained at $25{ }^{\circ} \mathrm{C}$ on either a Bruker $700 \mathrm{MHz}$ Spectrometer, Bruker DRX $600 \mathrm{MHz}$ Spectrometer, Bruker ARX $400 \mathrm{MHz}$ Spectrometer or Bruker ARX $300 \mathrm{MHz}$ Spectrometer. Chemical shifts are reported relative to $\mathrm{SiMe}_{4}$ and referenced to the residual solvent signal $\left({ }^{1} \mathrm{H},{ }^{13} \mathrm{C}\left\{{ }^{1} \mathrm{H}\right\}\right)$. NMR spectra were analyzed using either TopSpin 4.0.1 or MestReNova 6.0.2-5475 software. Chemical shifts are reported in ppm and coupling constants as scalar values in $\mathrm{Hz}$. The conventional abbreviations were used as follows: $s$ (singlet), $d$ (doublet), $t$ (triplet), q (quartet), dd (doublet of doublets), $m$ (multiplet), br (broad). Absorption measurements were recorded with a Cary 5000 UV-Vis-NIR Spectrophotometer from Agilent Technologies. Recordings were obtained at $25{ }^{\circ} \mathrm{C}$ and taken with the instrument operating in dual beam mode and referenced to methanol. All absorption experiments were conducted in quartz cuvettes $(1 \mathrm{~cm} \times 1 \mathrm{~cm})$ equipped with a Teflon seal. 
2. UV-Visible Absorbance Spectra of Starting Materials

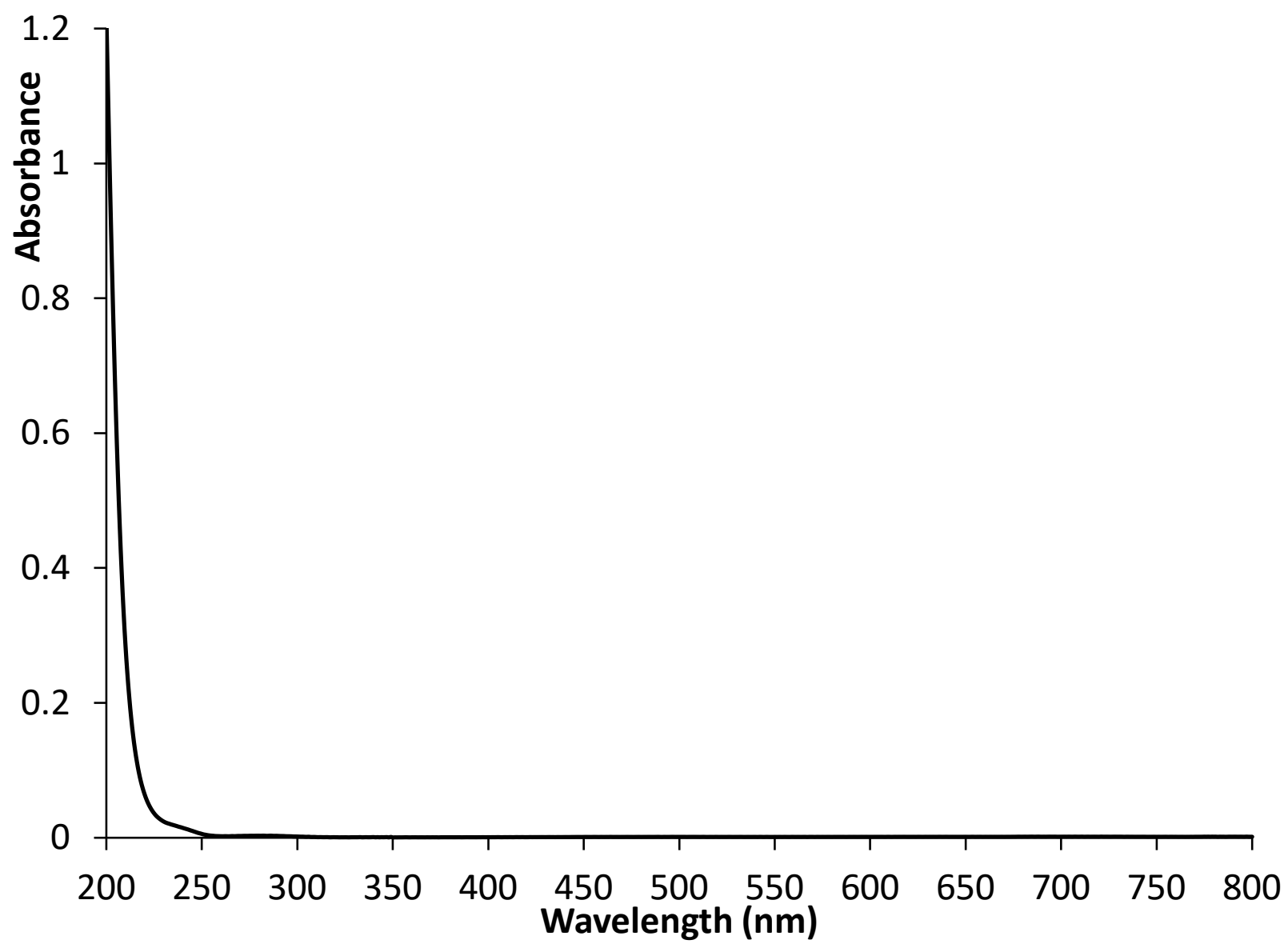

Figure S1 - UV-visible absorbance spectrum of $2.0 \times 10^{-3} \mathrm{M} \mathrm{MeOH}$ solution of putrescine. 


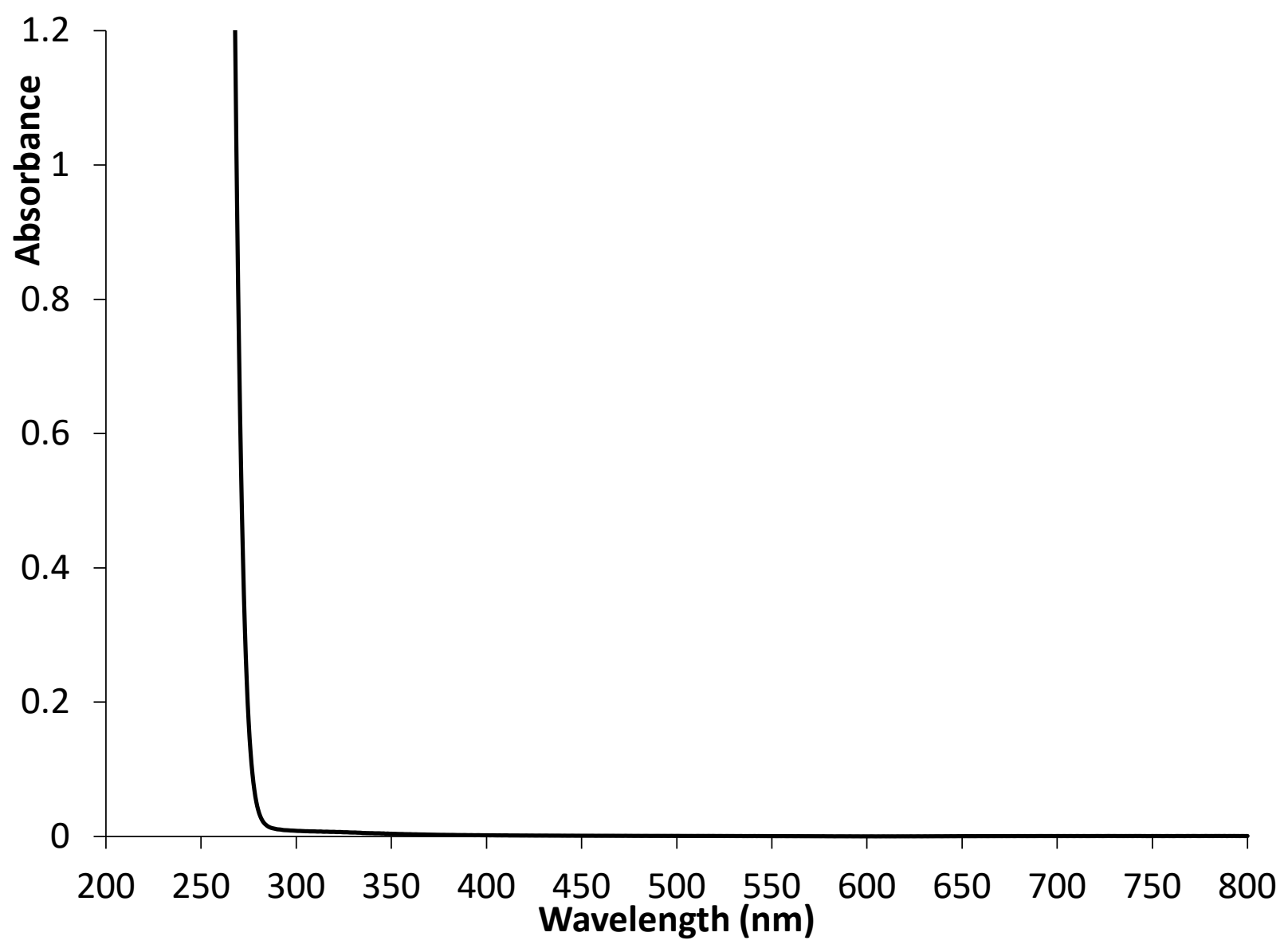

Figure S2 - UV-visible absorbance spectrum of $2.0 \times 10^{-3} \mathrm{M} \mathrm{MeOH}$ solution of genipin 


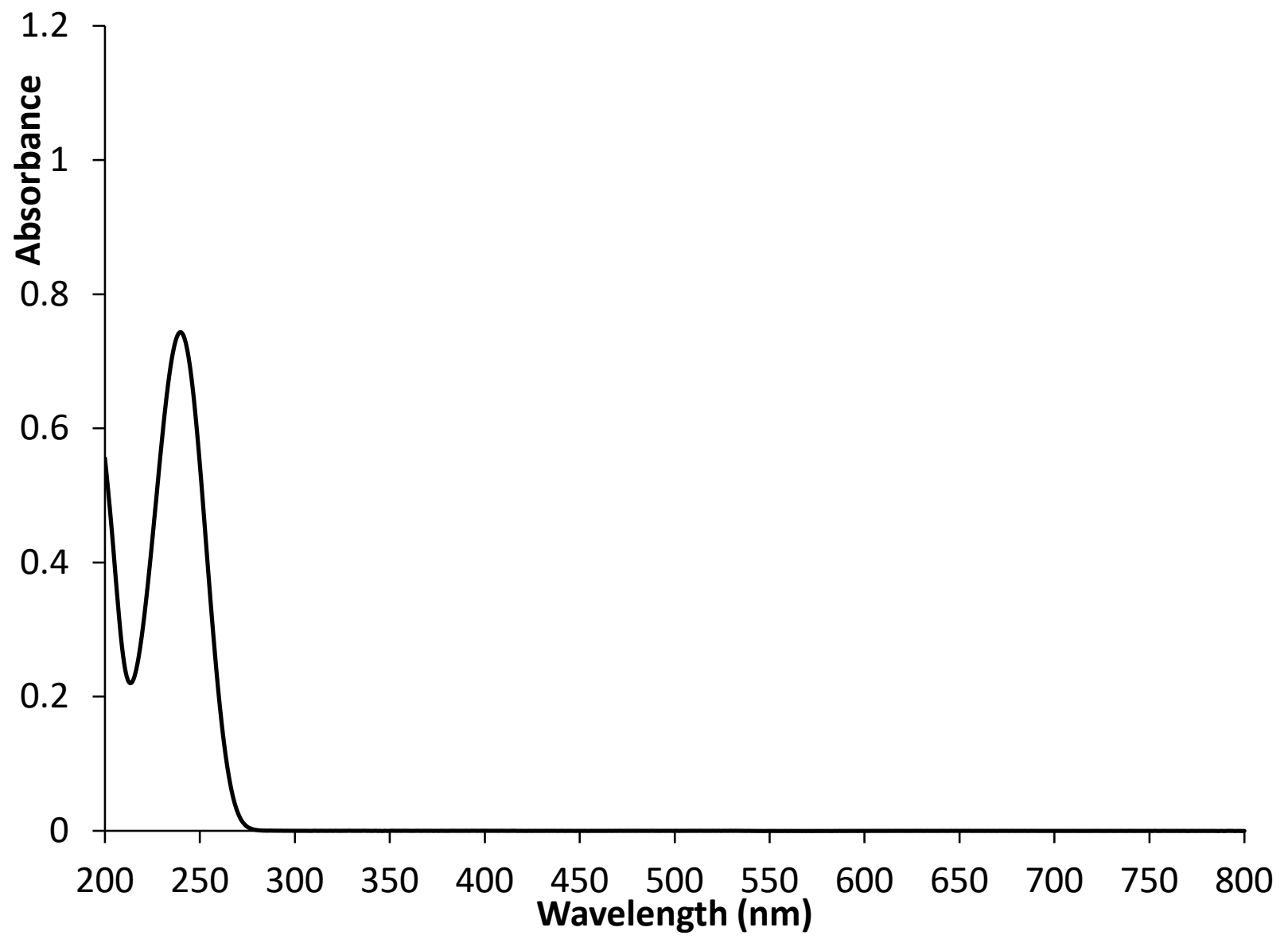

Figure S3 - UV-visible absorbance spectrum of $8.0 \times 10^{-5} \mathrm{M} \mathrm{MeOH}$ solution of genipin 


\section{UV-Visible Absorbance Spectra - Reaction of Amines with Genipin in Solution}

\section{General Procedure}

Genipin ( $22.6 \mathrm{mg}, 0.10 \mathrm{mmol}$ ) was dissolved in $45.0 \mathrm{~mL}$ methanol in a single-neck round-bottom flask by stirring for five minutes with a magnetic stirrer. For 1:1 solutions of genipin:amine , 0.10 $\mathrm{mmol}$ of the amine of choice was added to a separate pre-weighed vial and rinsed into the genipin solution with a further $5.0 \mathrm{~mL}$ methanol to prepare $50.0 \mathrm{~mL}$ of solution of concentrations $2.0 \times 10^{-3} \mathrm{M}$ in genipin and amine. For solutions lower in concentration of amine, stock solutions $0.020 \mathrm{M}$ in amine were prepared in methanol, with appropriate volumes diluted with additional methanol as needed and added to the methanolic genipin solution, with total volumes made up to $50.0 \mathrm{~mL}$. Round-bottomed flasks containing solutions were left open to allow exposure to oxygen and stirred for up to 72 hours. Measurement of UV-visible absorbances were carried out by decanting solutions into a graduated cylinder at time of measurement, making up the total volume to $50.0 \mathrm{~mL}$ with methanol to compensate for evaporation, decanting back into the roundbottom flask and stirring for 5 minutes to achieve homogeneity. Samples were then transferred into a quartz cuvette for analysis. Single-neck (1" diameter) round-bottom flasks were chosen as reaction vessels after verifying that rates of evaporation of stirred methanol at room temperature ensured that solution volumes never fell below $40 \mathrm{~mL}$. Samples exhibiting peak absorbances $>1$ in the visible range were diluted by $2 \mathrm{X}, 5 \mathrm{X}$, or $10 \mathrm{X}$ and absorbance spectra measured at these concentrations. 


\subsection{Reactions of Benzylamines with Genipin}

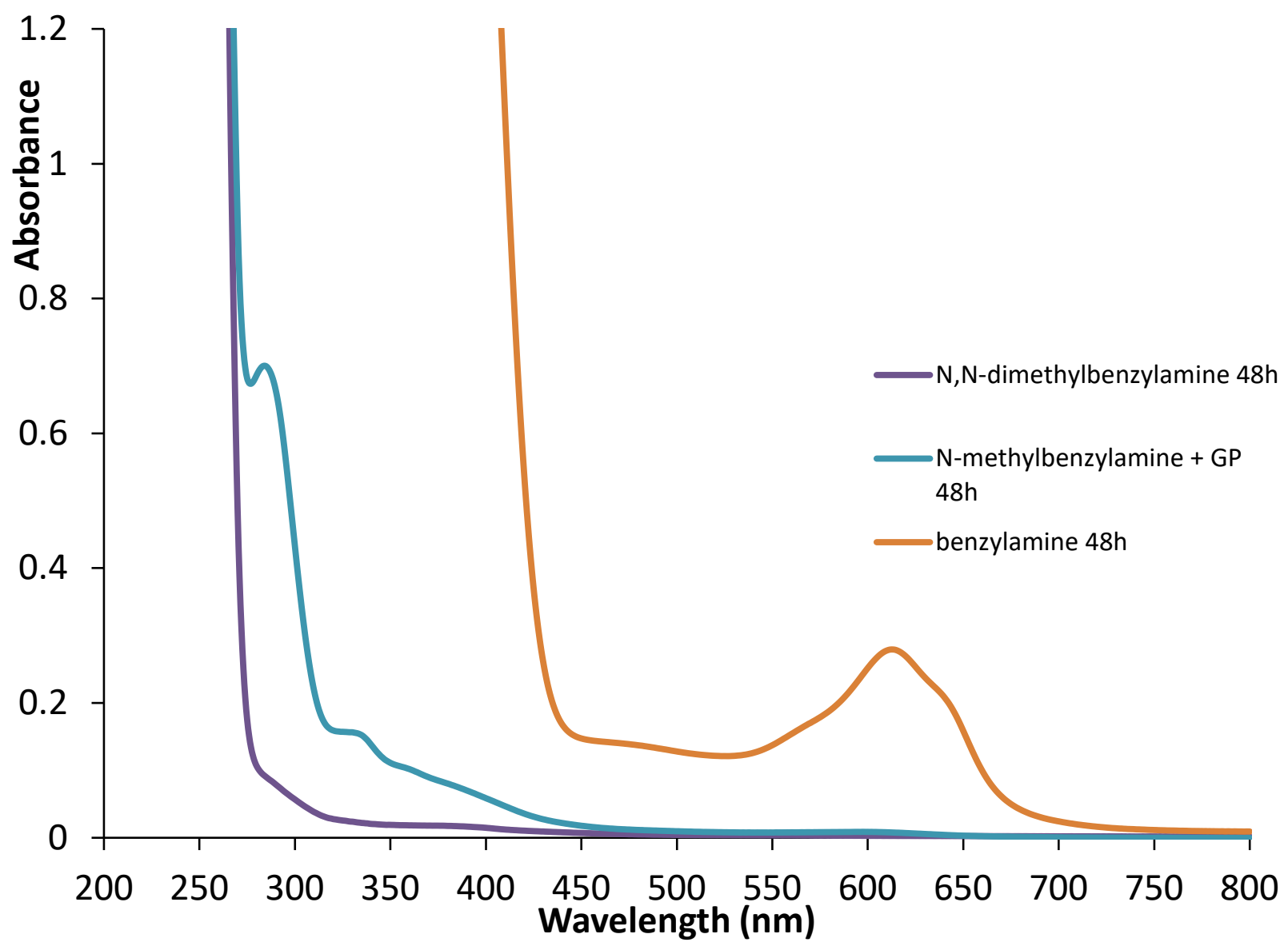

Figure S4 - UV-visible absorbance spectra of $2.0 \times 10^{-3} \mathrm{M}$ solutions of benzylamine, $\mathrm{N}$ methylbenzylamine, or $\mathrm{N}, \mathrm{N}$-dimethylbenzylamine solutions after reaction with genipin at $\left[2.0 \times 10^{-3} \mathrm{M}\right]$ after $48 \mathrm{~h}$ 


\subsection{Reactions of Genipin with Putrescine (1,4-Diaminobutane)}

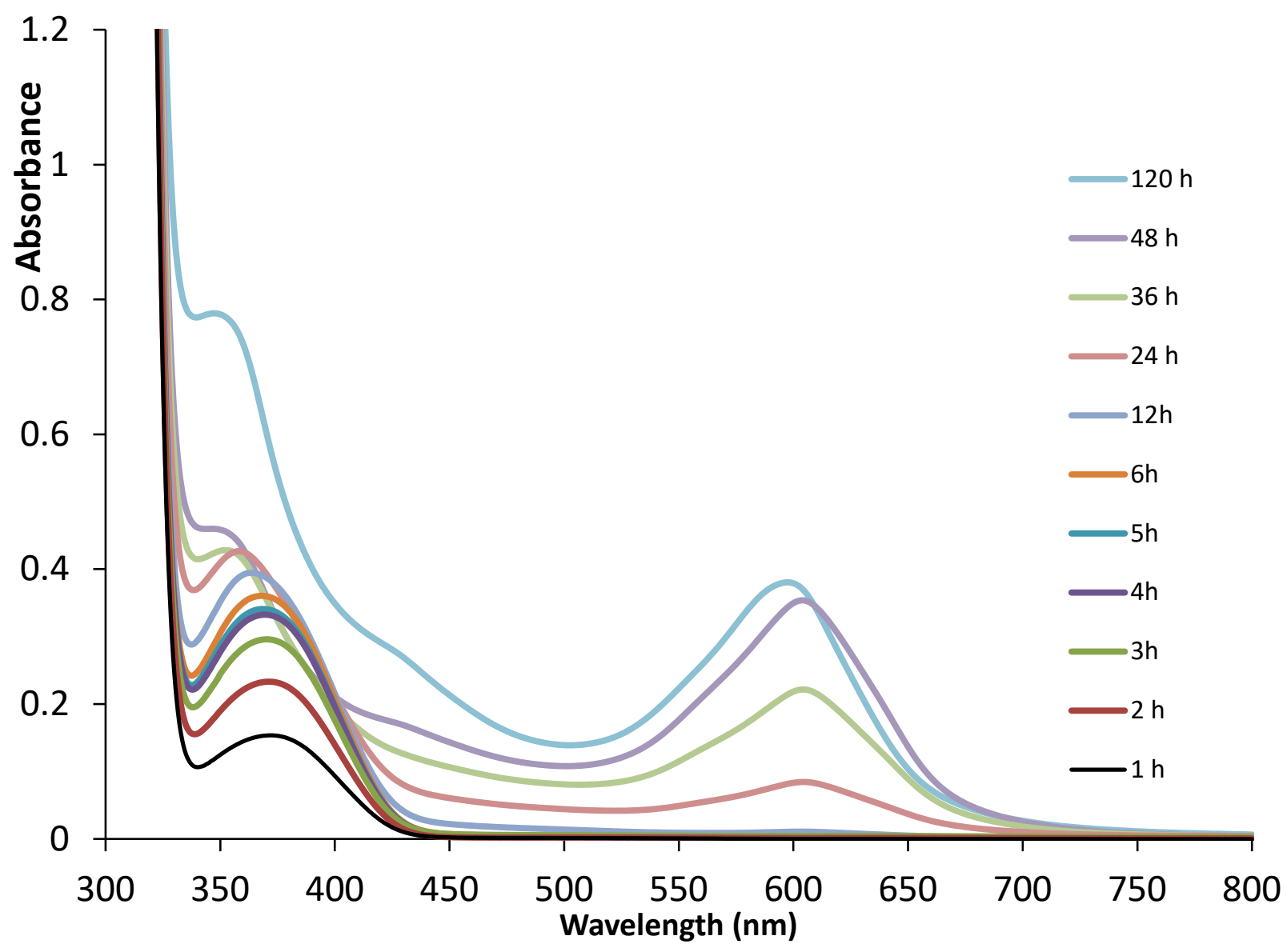

Figure S5 - UV-visible absorbance spectra of a $\mathrm{MeOH}$ solution $2.0 \times 10^{-3} \mathrm{M}$ in both genipin and putrescine monitored over 1-120 h. 
Exclusion of Oxygen from Reaction - The general procedure was slightly modified to utilize a Schlenk flask or glovebox techniques to ensure an $\mathrm{N}_{2}$ atmosphere throughout the reaction.

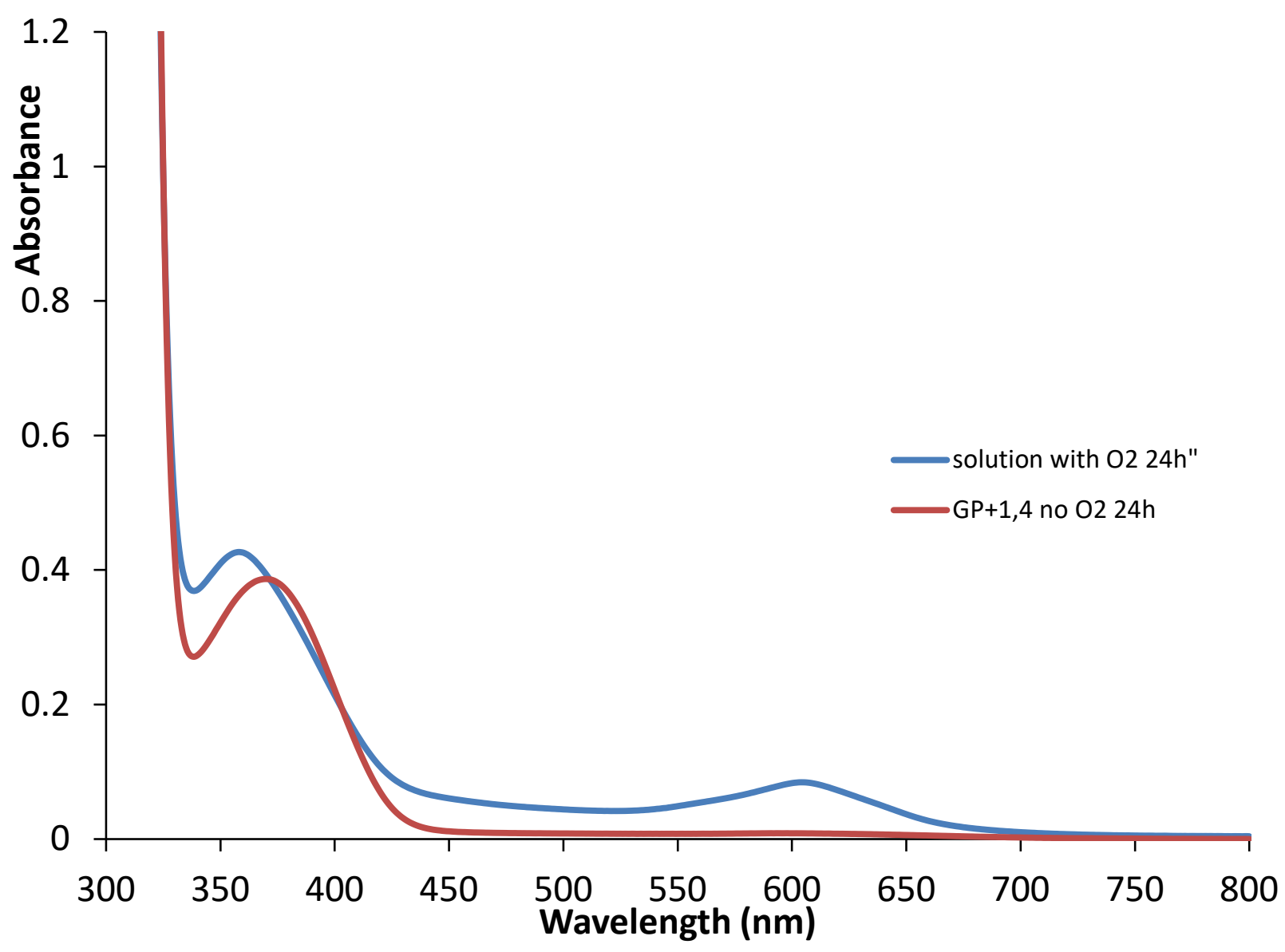

Figure S6 - UV-visible absorbance spectra of $\mathrm{MeOH}$ solution $2.0 \times 10^{-3} \mathrm{M}$ in both genipin and putrescine after $24 \mathrm{~h}$ in the presence and absence of $\mathrm{O}_{2}$ 


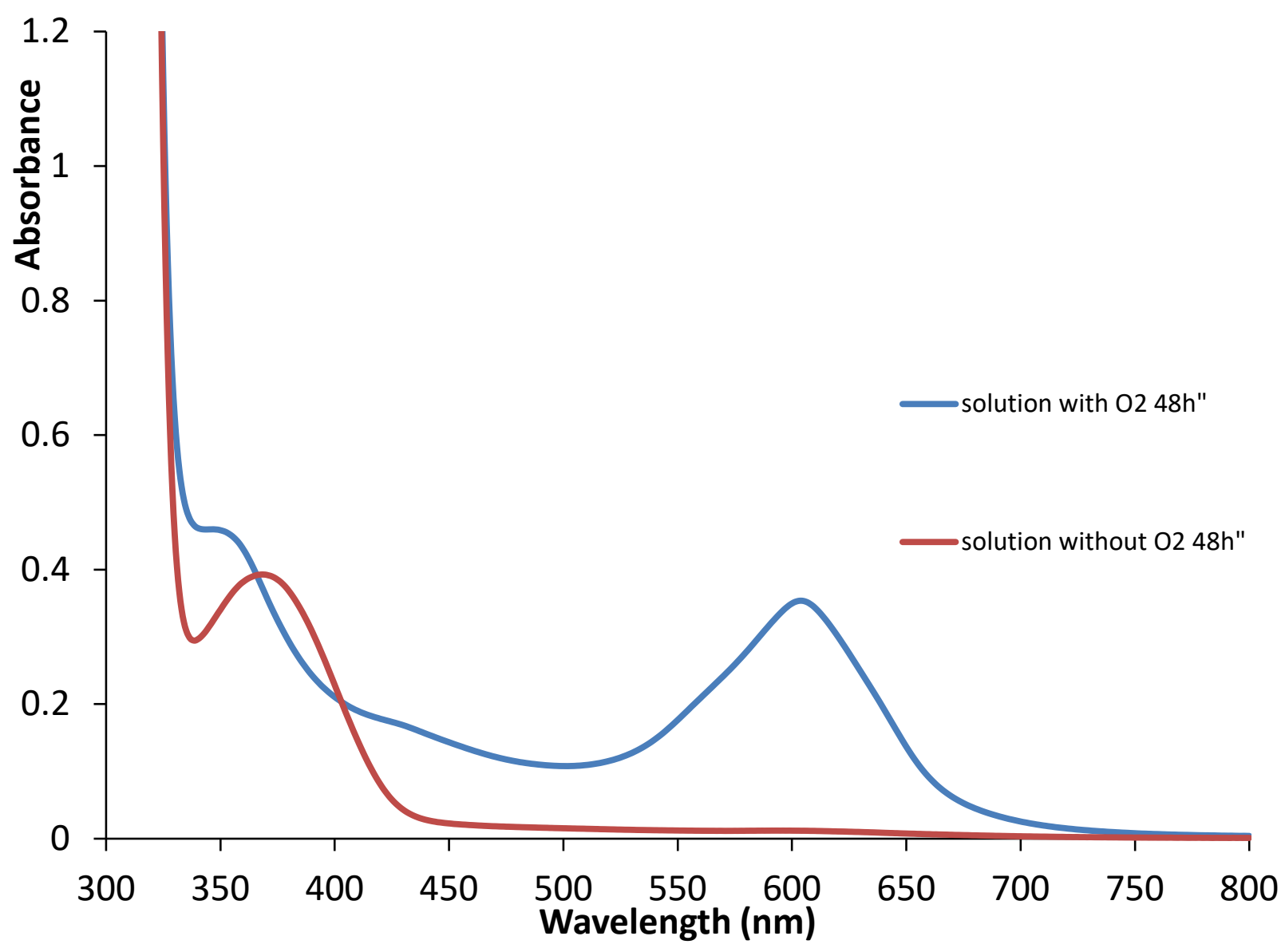

Figure S7 - UV-Visible absorbance spectra of $\mathrm{MeOH}$ solution $2.0 \times 10^{-3} \mathrm{M}$ in both genipin and putrescine after $48 \mathrm{~h}$ in the presence and absence of $\mathrm{O}_{2}$ 


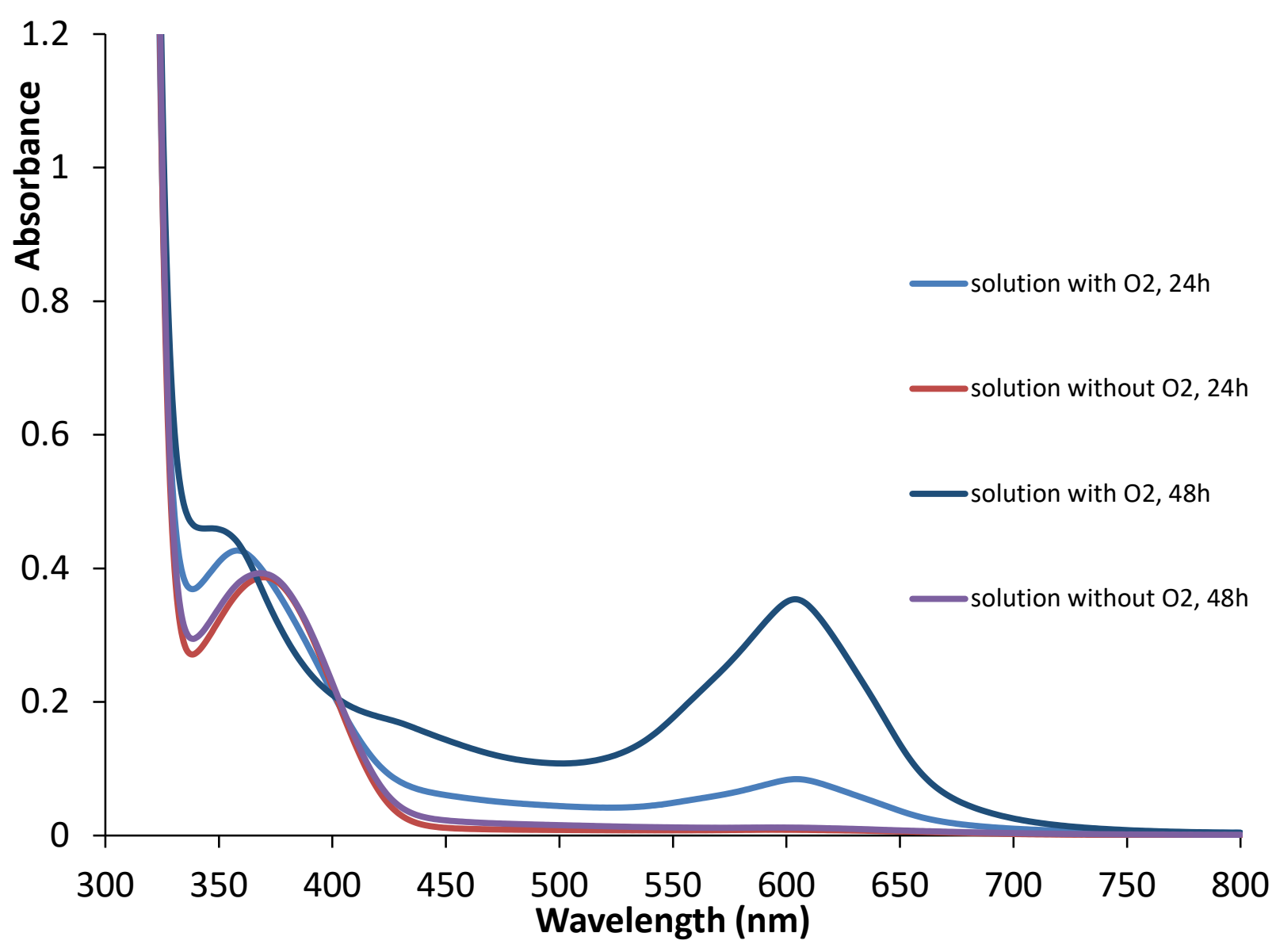

Figure S8 - Combined UV-visible absorbance spectra of $\mathrm{MeOH}$ solutions $2.0 \times 10^{-3} \mathrm{M}$ in both genipin and putrescine after $24-48 \mathrm{~h}$ in the presence and absence of $\mathrm{O}_{2}$ 


\subsection{Reaction of Genipin with Putrescine With Addition of Chemically-Generated Oxygen}

Under a nitrogen atmosphere inside a glove box, two portions of genipin ( $23 \mathrm{mg}, 0.10 \mathrm{mmol}$ ) were dissolved in $8.0 \mathrm{~mL}$ of de-gassed methanol in separate, single-neck $50 \mathrm{~mL}$ round-bottom flasks. Putrescine $(8.8 \mathrm{mg}, 0.10 \mathrm{mmol})$ was added to two separate, pre-weighed vials and rinsed into the genipin solutions with a further $2.0 \mathrm{~mL}$ methanol, to prepare two $10.0 \mathrm{~mL}$ solutions of concentrations $1.0 \times 10^{-2} \mathrm{M}$ in genipin and putrescine. A magnetic stir bar was added to both flasks, both were stoppered and allowed to stir for 24 hours.

After 24 hours, both reaction mixtures appeared reddish-golden in colour. Stirring was stopped for both solutions. The stopper was removed from one round bottom flask and a 1-dram vial containing 3-5 mg (0.02-0.03 mmol) $\mathrm{KMnO}_{4}$ in $0.5 \mathrm{~mL}$ distilled $\mathrm{H}_{2} \mathrm{O}$ was placed inside such that the mouth of the vial rested well above the level of the genipin-amine solution. A septum was placed over the mouth of the round bottom flask. $10 \mu \mathrm{L}$ of a $3 \%$ aqueous $\mathrm{H}_{2} \mathrm{O}_{2}$ solution ( $9 \mu \mathrm{mol}$ $\mathrm{H}_{2} \mathrm{O}_{2}$ ) was added to the $\mathrm{KMnO}_{4}$ solution via syringe to evolve $9 \mu \mathrm{mol} \mathrm{O}_{2}$ gas $(0.2 \mathrm{~mL}$, equating to $0.5 \%$ headspace volume, $5000 \mathrm{ppm}$ ) according to the balanced reaction $\mathbf{S 1}$ and calculation $\mathbf{S 2}$ :

Equation $\mathrm{S} 1: 3 \mathrm{H}_{2} \mathrm{O}_{2}+2 \mathrm{KMnO}_{4} \rightarrow 2 \mathrm{MnO}_{2}+2 \mathrm{KOH}+2 \mathrm{H}_{2} \mathrm{O}+3 \mathrm{O}_{2}$

Equation S2: $V_{02}=n R T / P=(9 \times 10-6 \mathrm{~mol})(0.08206 \mathrm{~L} \mathrm{~atm} / \mathrm{mol} \mathrm{K})(298 \mathrm{~K}) / 1.0 \mathrm{~atm}=2.0 \times 10^{-4} \mathrm{~L}$ 


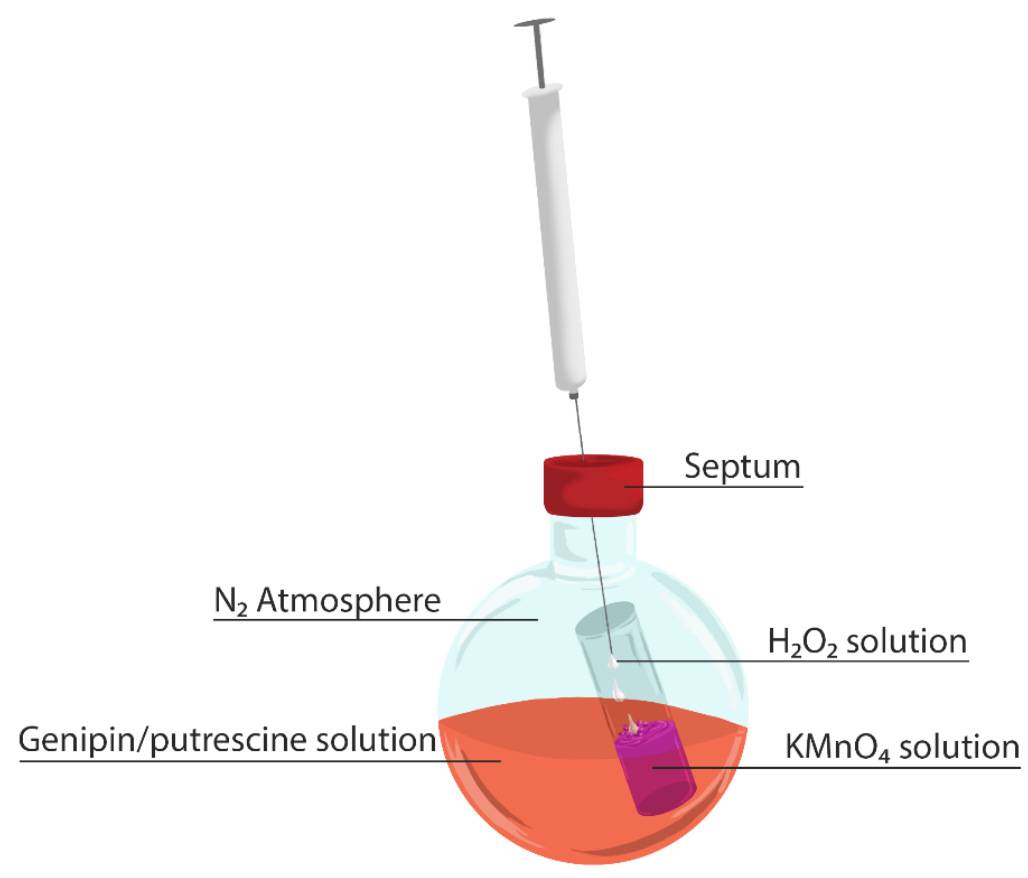

The $\mathrm{O}_{2}$ generated by this reaction was allowed to diffuse into the headspace of the flask for 24 hours. After about 1 hour, the solution with the oxygen added to the headspace appeared slightly darker. After 24 hours, the solution with the $\mathrm{O}_{2}$ added to the headspace appeared the deep blue typical of genipin reaction with amines.

Measurement of UV-visible absorbances were carried out by decanting solutions into a graduated cylinder, making up the total volume to $10.0 \mathrm{~mL}$ with methanol to compensate for evaporation, decanting back into the round-bottom flask and stirring for 5 minutes to achieve homogeneity. Samples were removed from each solution, diluted by a factor of $5 X$, decanted into sealable quartz cuvettes to prevent additional $\mathrm{O}_{2}$ exposure prior to measurement, and sealed. Cuvettes were then removed from the glove box and UV-visible absorption spectra obtained (Figure S9). 


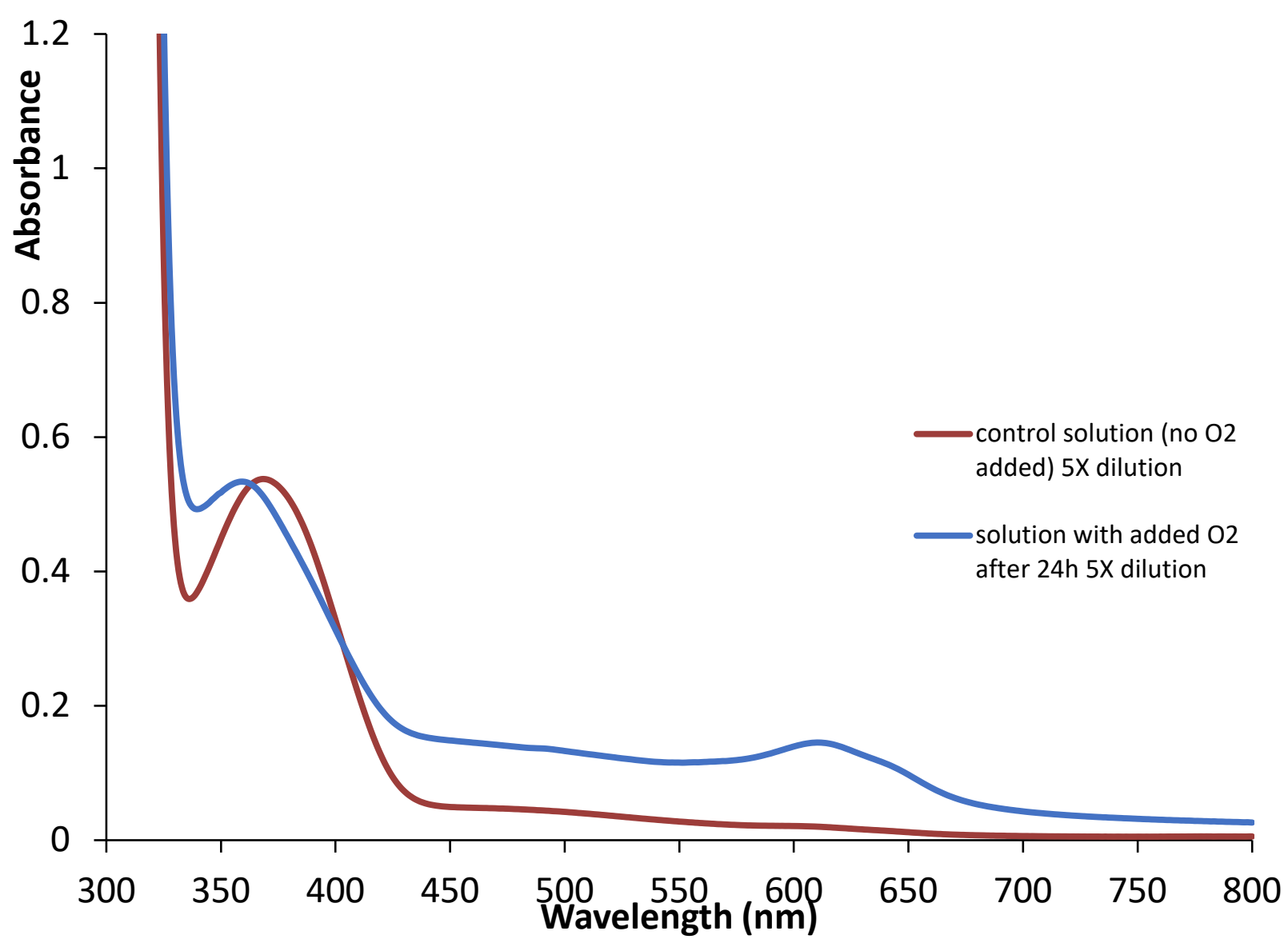

Figure S9 - UV-visible absorbance spectra of $\mathrm{MeOH}$ solutions $1.0 \times 10^{-2} \mathrm{M}$ in both genipin and putrescine after reaction under $\mathrm{N}_{2}$ atmosphere, with the spectrum in red representing absorbance of the solution with no added $\mathrm{O}_{2}$ and the spectrum in blue after $24 \mathrm{~h}$ in the presence of $5000 \mathrm{ppm} \mathrm{O} \mathrm{O}_{2}$ added by reaction of $\mathrm{H}_{2} \mathrm{O}_{2}$ and $\mathrm{KMnO}_{4}$ solutions. 


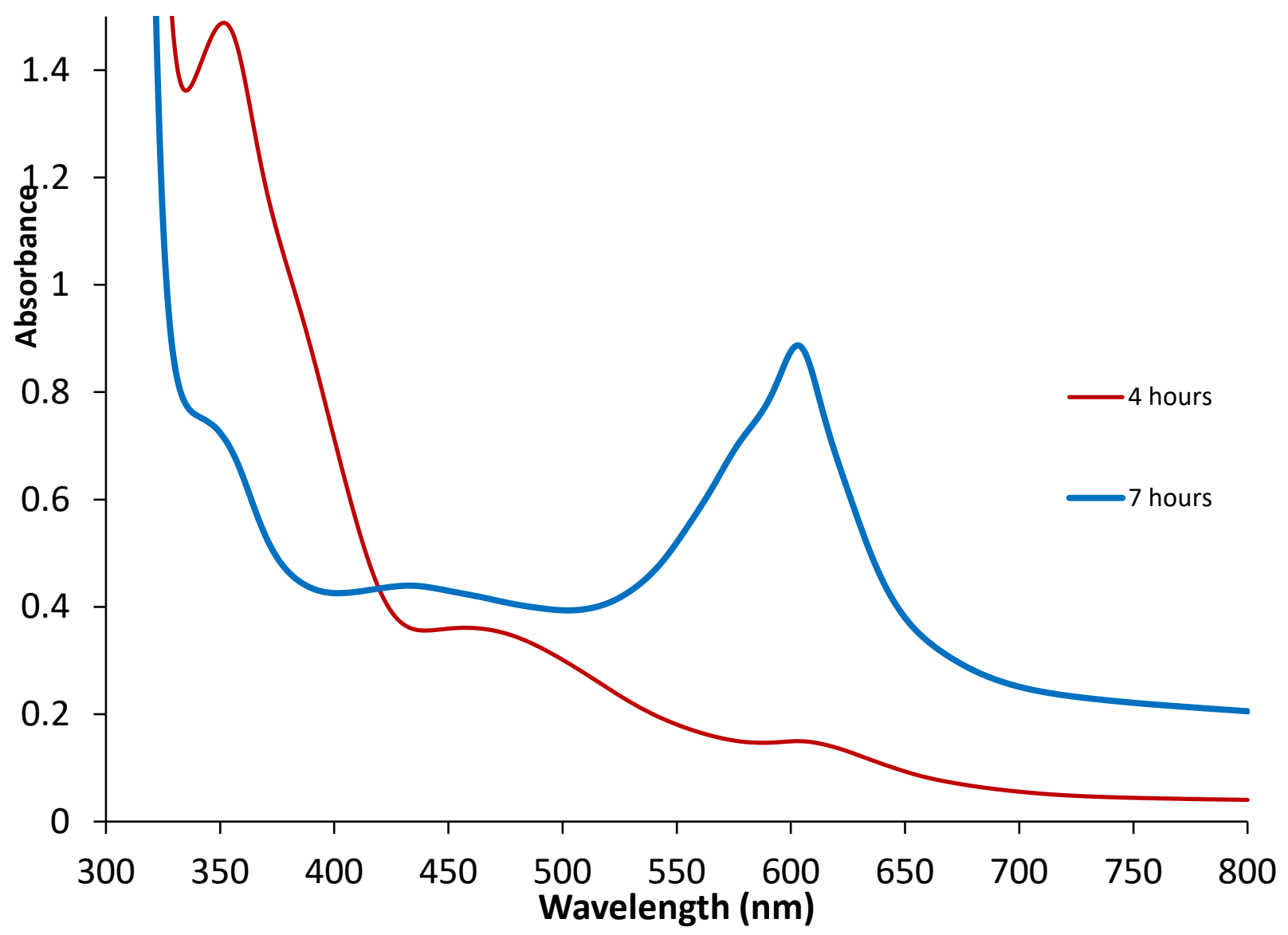

Figure S10 - UV-visible absorbance spectra of $\mathrm{MeOH}$ reaction mixtures $0.20 \mathrm{M}$ in both genipin and putrescinein $\mathrm{MeOH}$ after $4 \mathrm{~h} 7 \mathrm{~h}$ reaction times (diluted at time of measurement to $2.0 \times 10^{-3} \mathrm{M}$ ). 


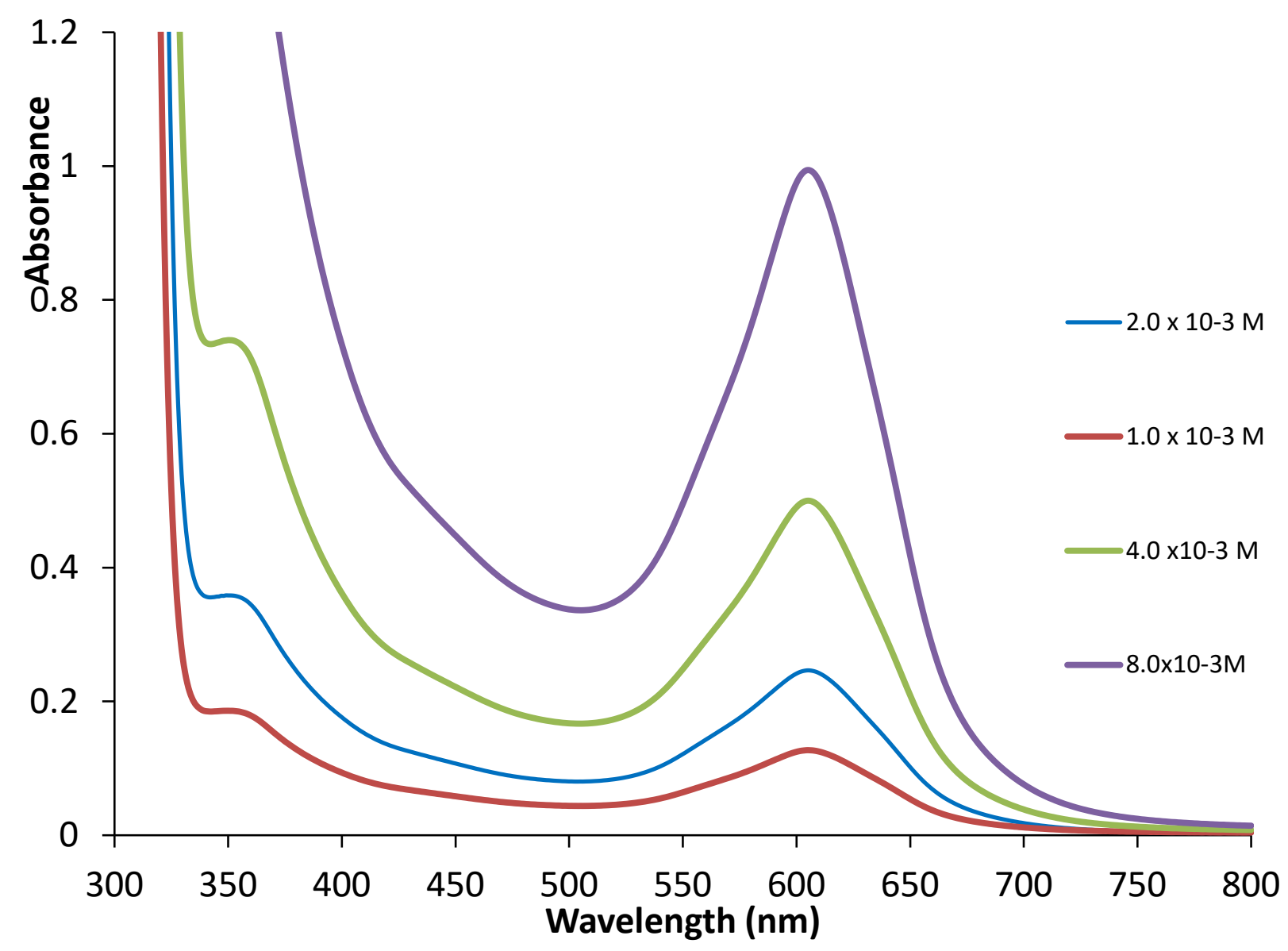

Figure S11 - UV-visible absorbance spectra of reaction mixtures $2.0 \times 10^{-3} \mathrm{M}$ in both genipin and putrescine in $\mathrm{MeOH}$ after $48 \mathrm{~h}$. Samples run at varying concentrations 


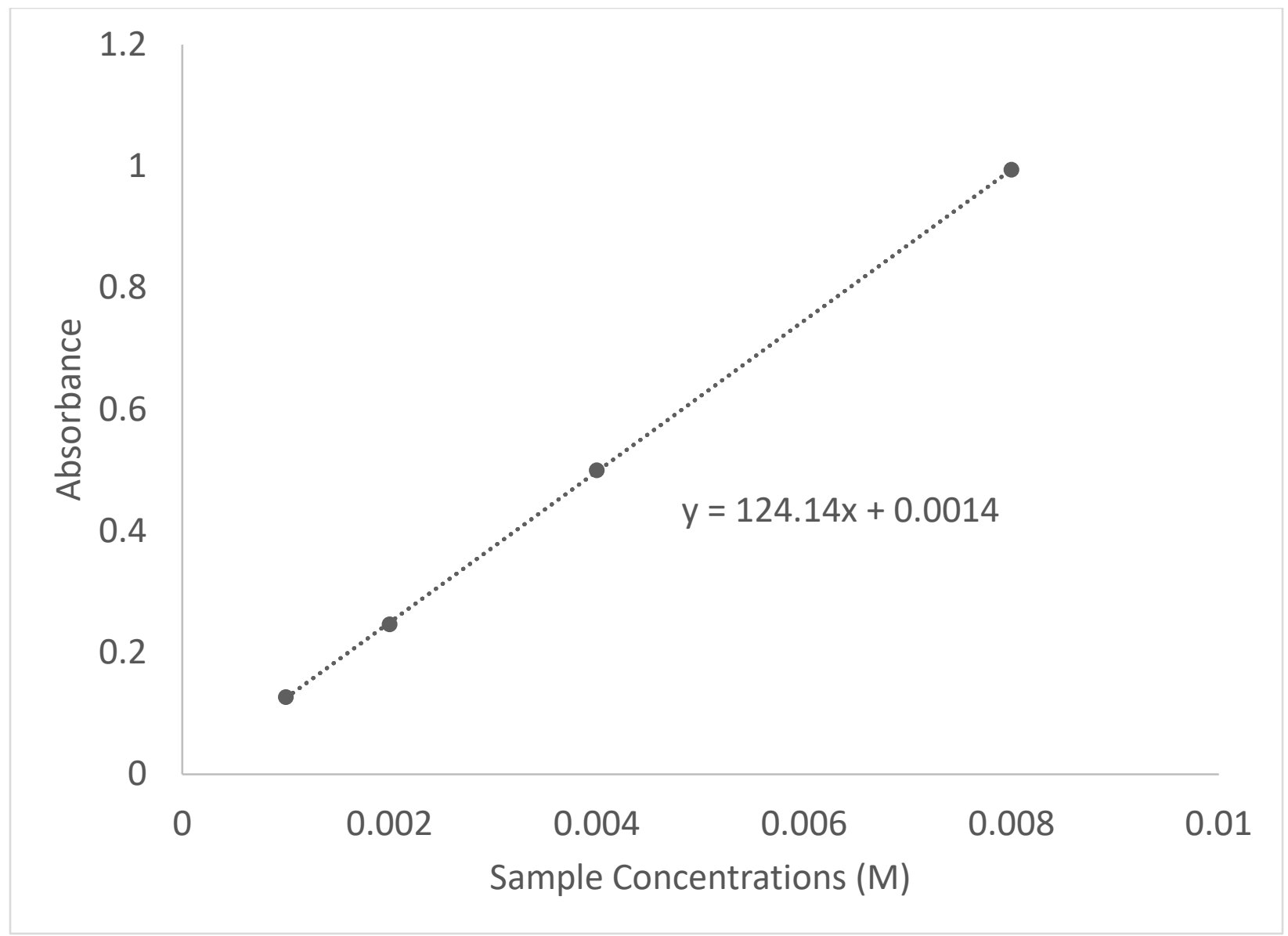

Figure S12 - UV-visible absorbances at $I_{\max }=605 \mathrm{~nm}$ for varying dilutions from a stock solution of concentration $2.0 \times 10^{-3} \mathrm{M}$ in both genipin and putrescine in $\mathrm{MeOH}$ after $48 \mathrm{~h}$

Molar Absorptivity:

$\varepsilon=\mathrm{A} / \mathrm{cl} \mathrm{I}=1.00 \mathrm{~cm} ; \mathrm{A} / \mathrm{c}=124.14 \mathrm{~L} / \mathrm{mol}$ thus $\varepsilon=1.2 \times 10^{2} \mathrm{~L} / \mathrm{mol} \mathrm{cm}$ 


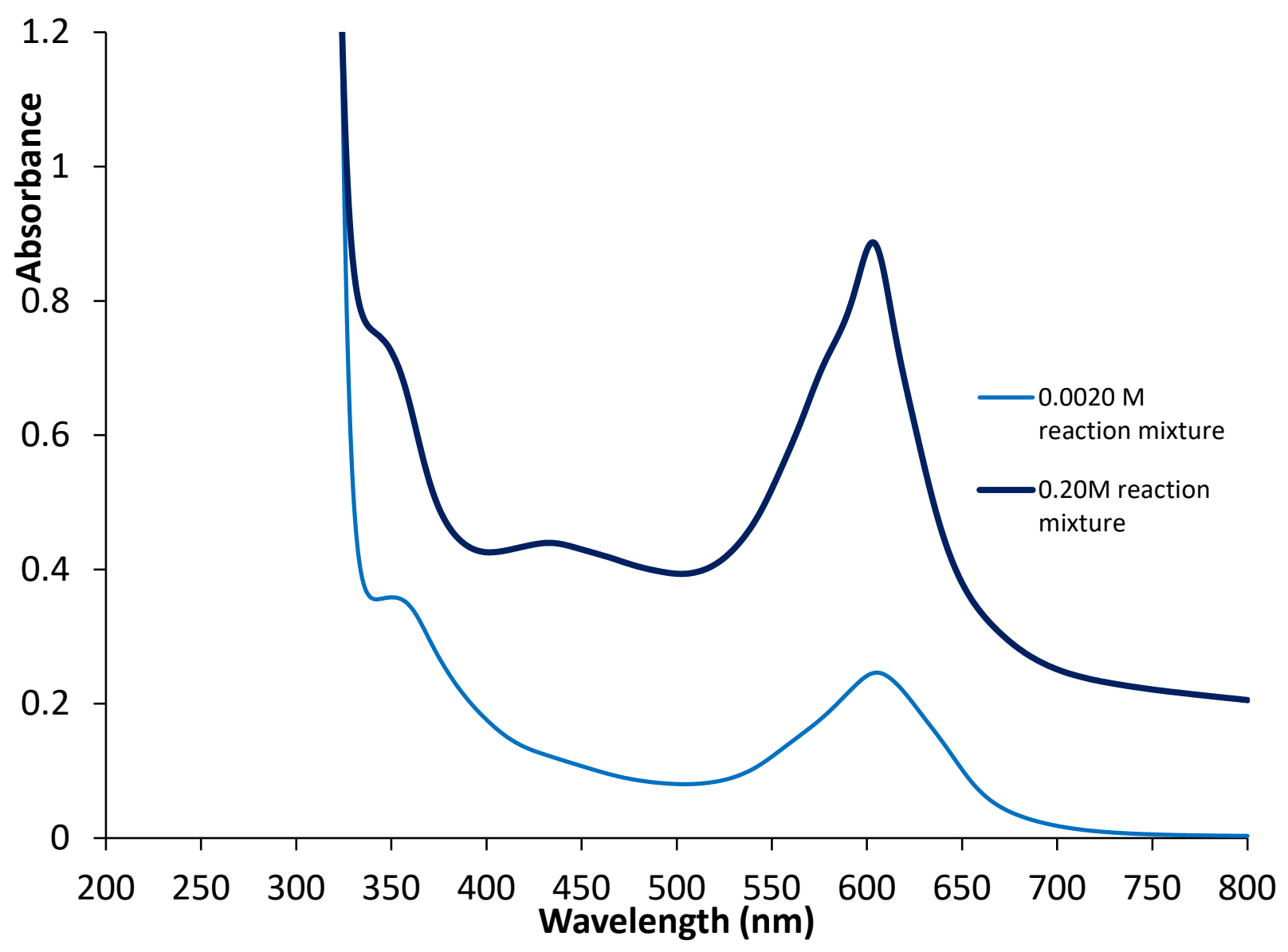

Figure S13 - Comparison of UV-visible absorbance spectra of reaction mixtures $2.0 \times 10^{-3} \mathrm{M}$ in both genipin and putrescine and $2.0 \times 10^{-1} \mathrm{M}$ (this sample diluted $100 \mathrm{x}$ ) in $\mathrm{MeOH}$ After $48 \mathrm{~h}$. 


\subsection{Reactions of Genipin with Cadaverine (1,5-Diaminopentane)}

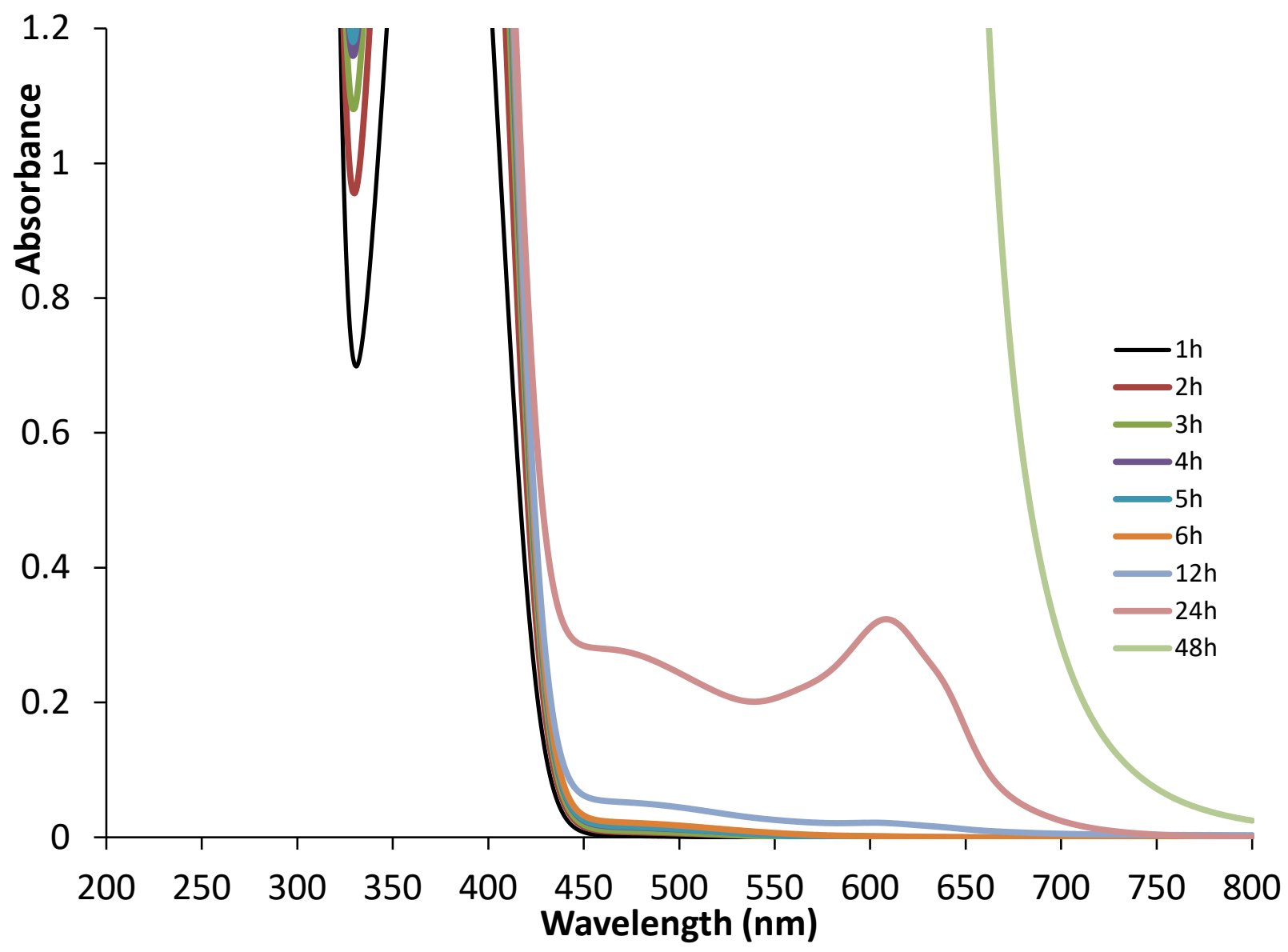

Figure S14 - UV-visible absorbance spectra of $\mathrm{MeOH}$ solutions $2.0 \times 10^{-3} \mathrm{M}$ in both cadaverine and genipin over $1-48 \mathrm{~h}$ 


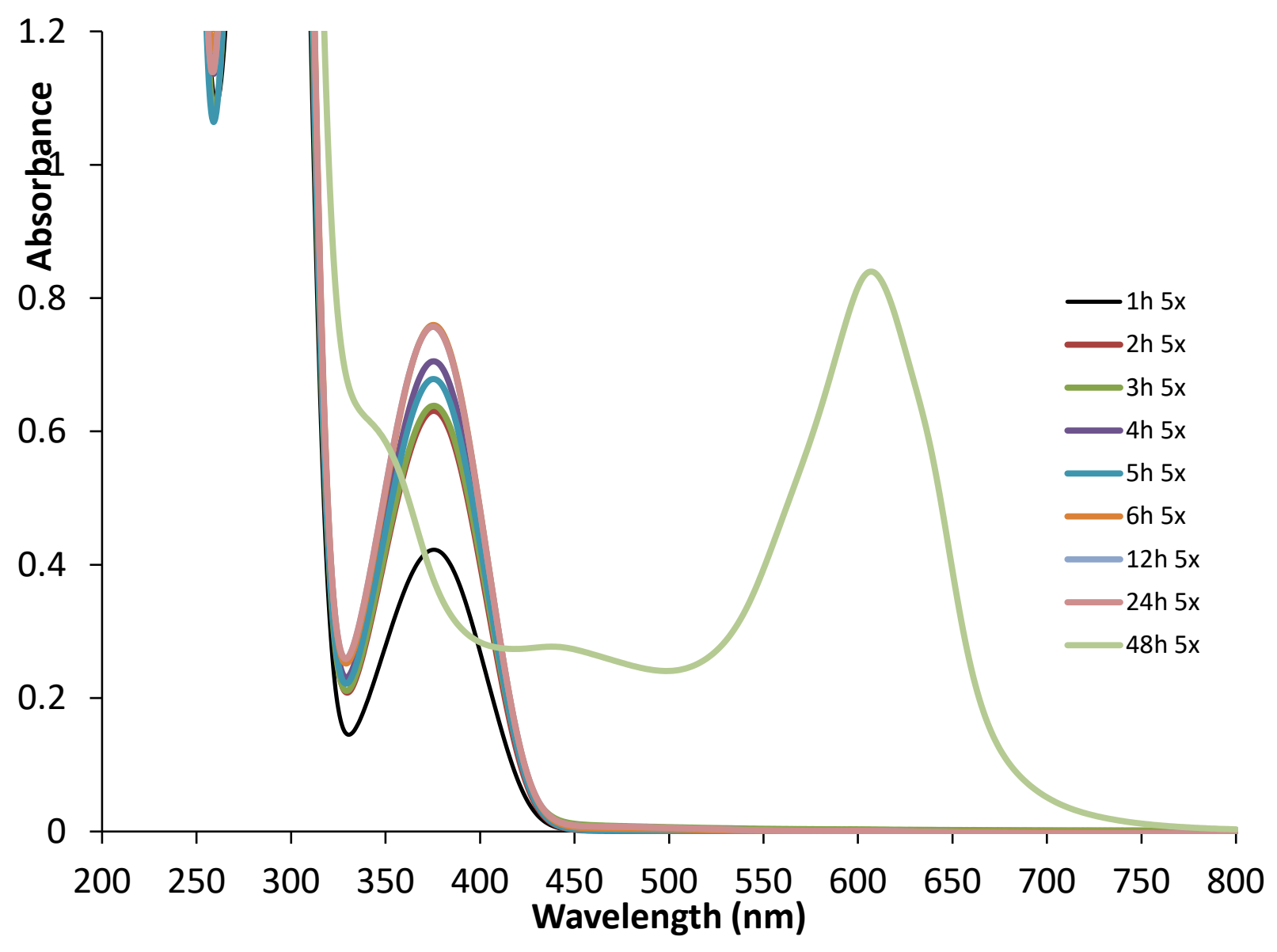

Figure S15 - UV-visible absorbance spectra of $\mathrm{MeOH}$ solutions $2.0 \times 10^{-3} \mathrm{M}$ in both cadaverine and genipin over diluted $5 \times 1-48 \mathrm{~h}$ 


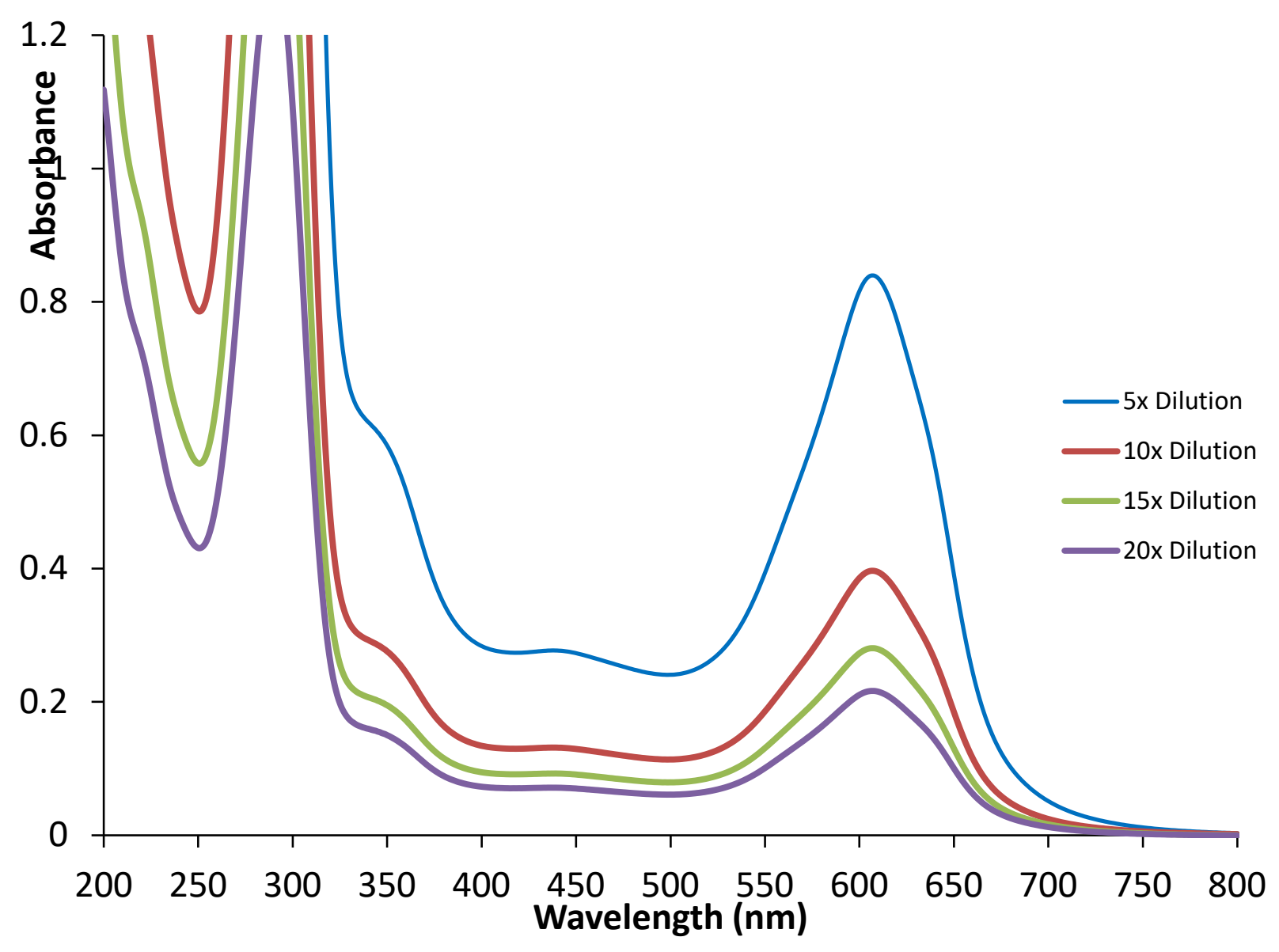

Figure S16 - UV-visible absorbance spectra of $\mathrm{MeOH}$ solutions $2.0 \times 10^{-3} \mathrm{M}$ in both cadaverine and genipin after $48 \mathrm{~h}$ diluted by various factors. 


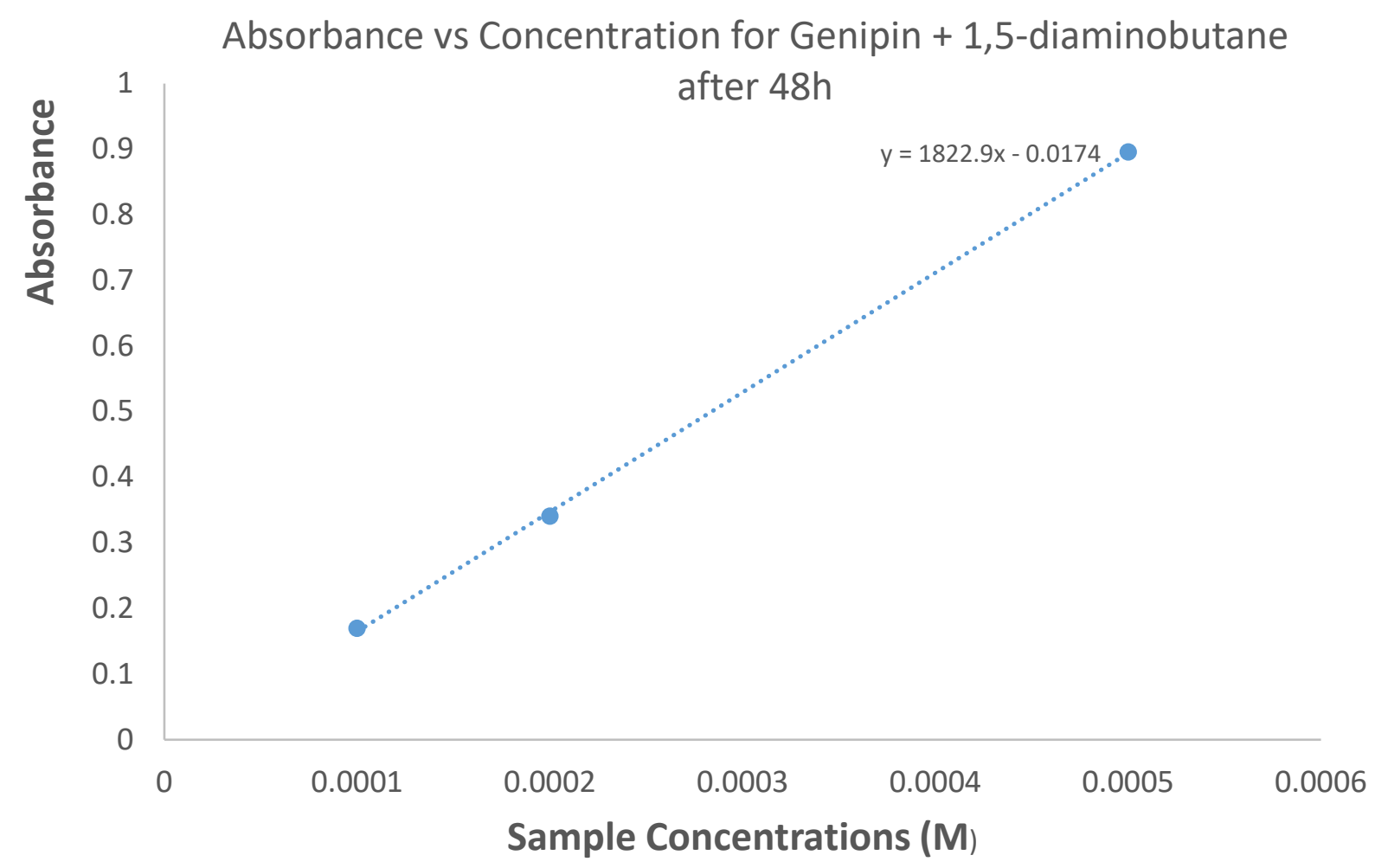

Figure S17 Plot of absorbance versus molar concentration of genipin/cadaverine solutions at $48 \mathrm{~h}$.

Molar Absorptivity:

$\varepsilon=\mathrm{A} / \mathrm{cl} \mathrm{I}=1.00 \mathrm{~cm} ; \mathrm{A} / \mathrm{c}=1822.9 \mathrm{~L} / \mathrm{mol}$ thus $\varepsilon=1.8 \times 10^{3} \mathrm{~L} / \mathrm{mol} \mathrm{cm}$ 


\subsection{Reactions of Genipin with Tyramine}

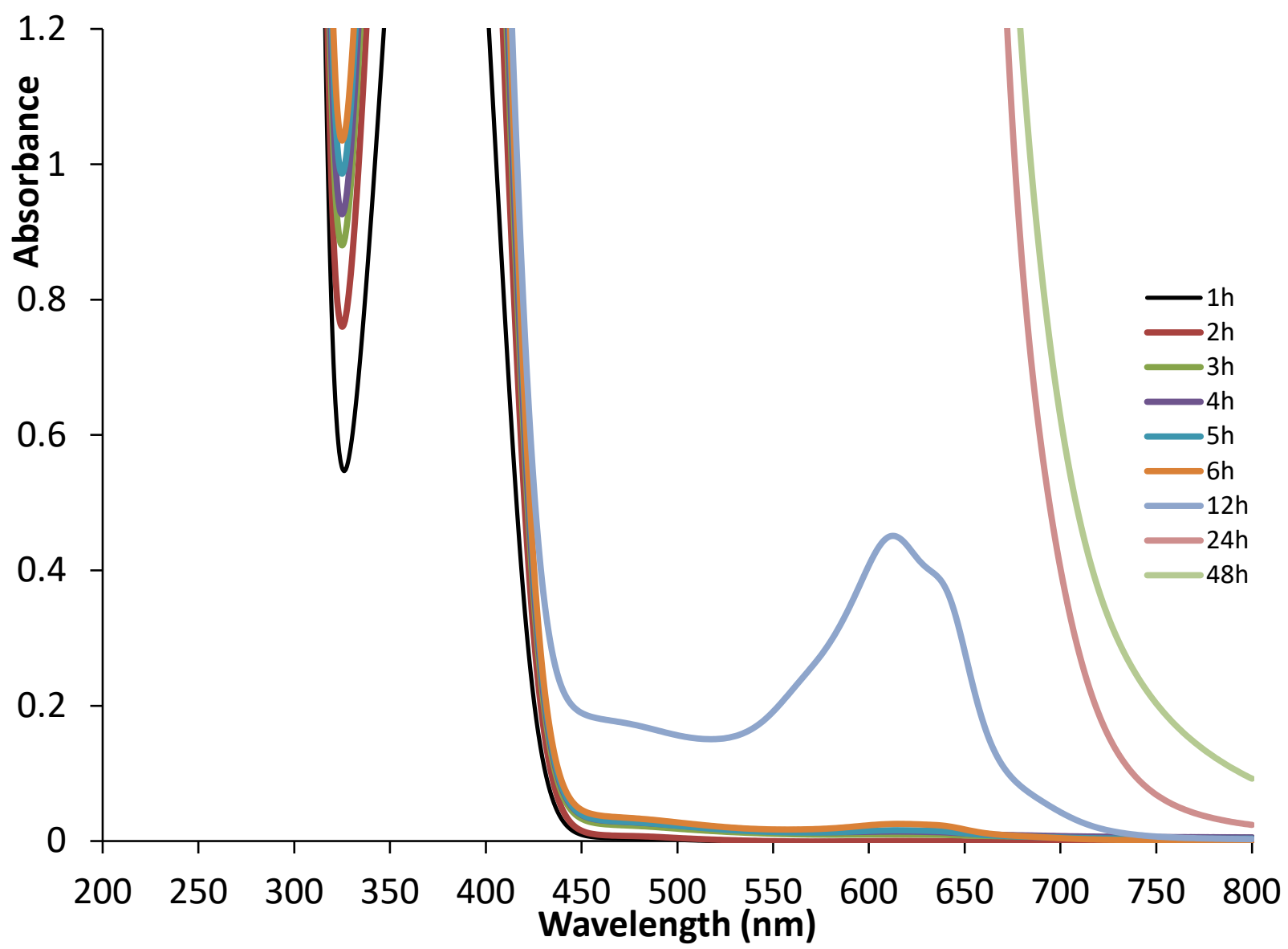

Figure S18 - UV-visible absorbance spectra of $\mathrm{MeOH}$ solutions $2.0 \times 10^{-3} \mathrm{M}$ in both genipin and tyramine $2.0 \times 10^{-3} \mathrm{M}$ over $1-48 \mathrm{~h}$ 


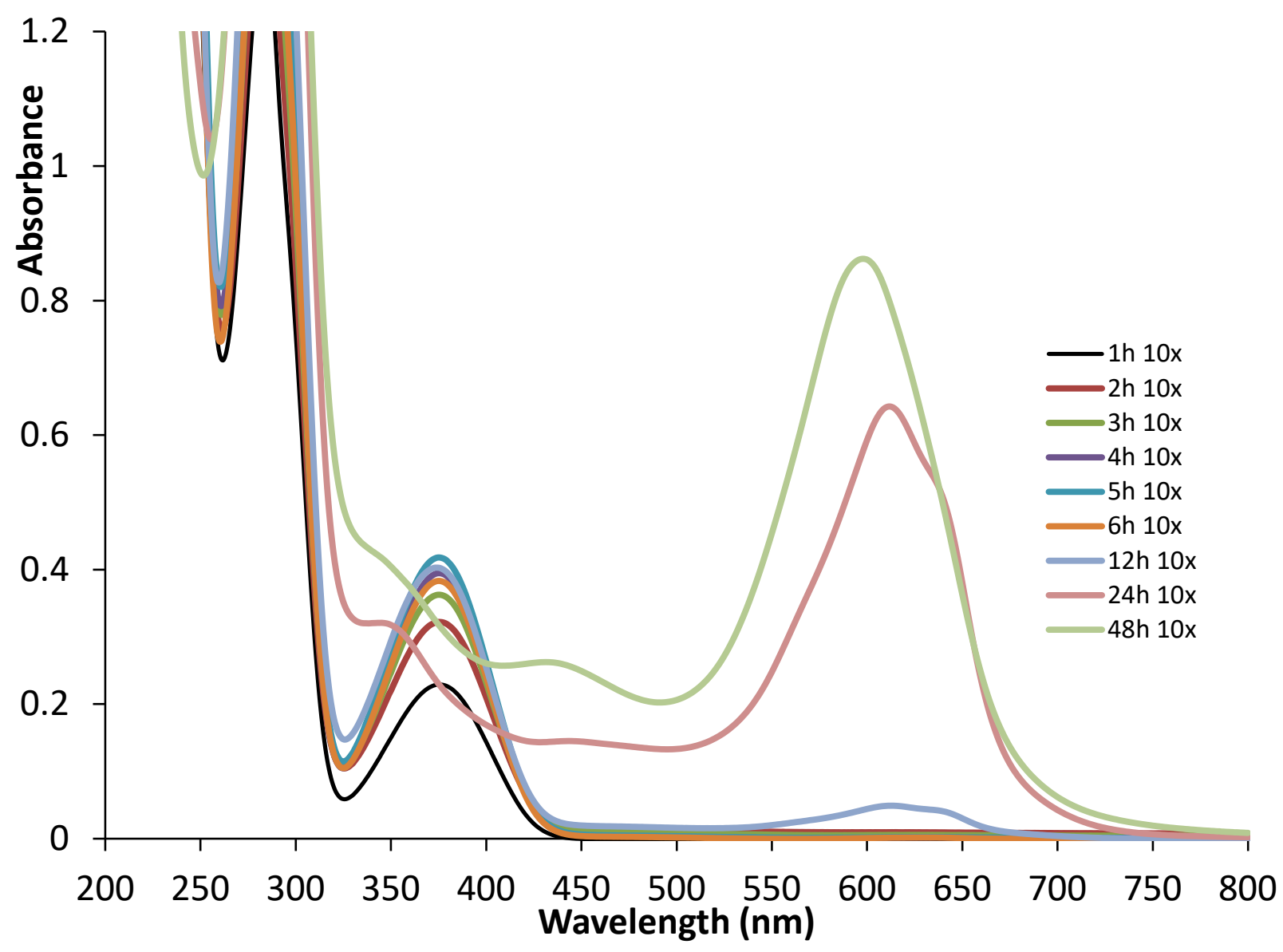

Figure S19 - UV-visible absorbance spectra of $\mathrm{MeOH}$ solutions $2.0 \times 10^{-3} \mathrm{M}$ in both genipin and tyramine $2.0 \times 10^{-3} \mathrm{M}$ over 1-48h Samples diluted $10 \mathrm{x}$ at time of measurement. 


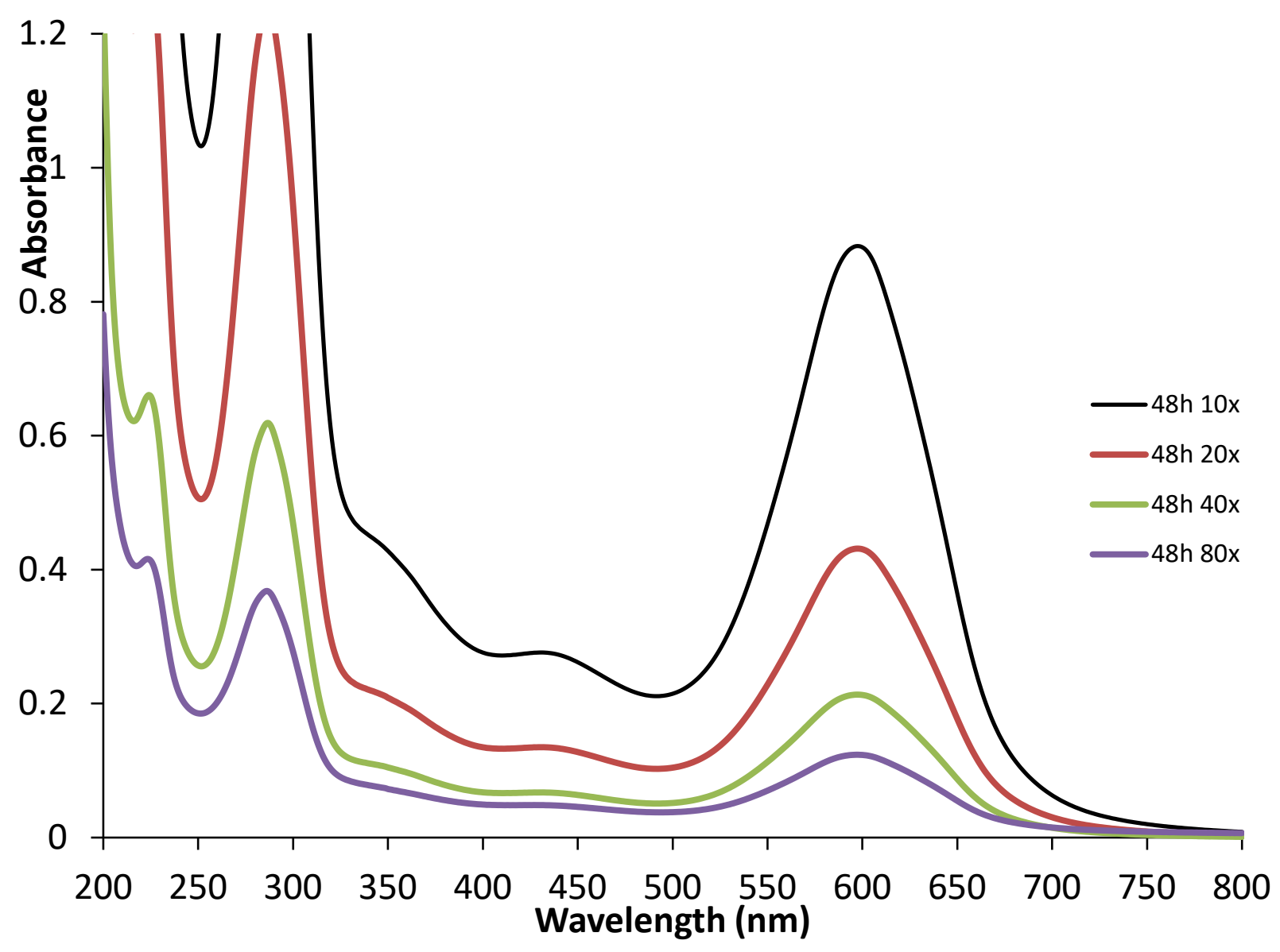

Figure S20 - UV-visible absorbance spectra of $\mathrm{MeOH}$ solutions $2.0 \times 10^{-3} \mathrm{M}$ in both genipin and tyramine $2.0 \times 10^{-3} \mathrm{M}$ over $1-48 \mathrm{~h}$ at various dilutions 


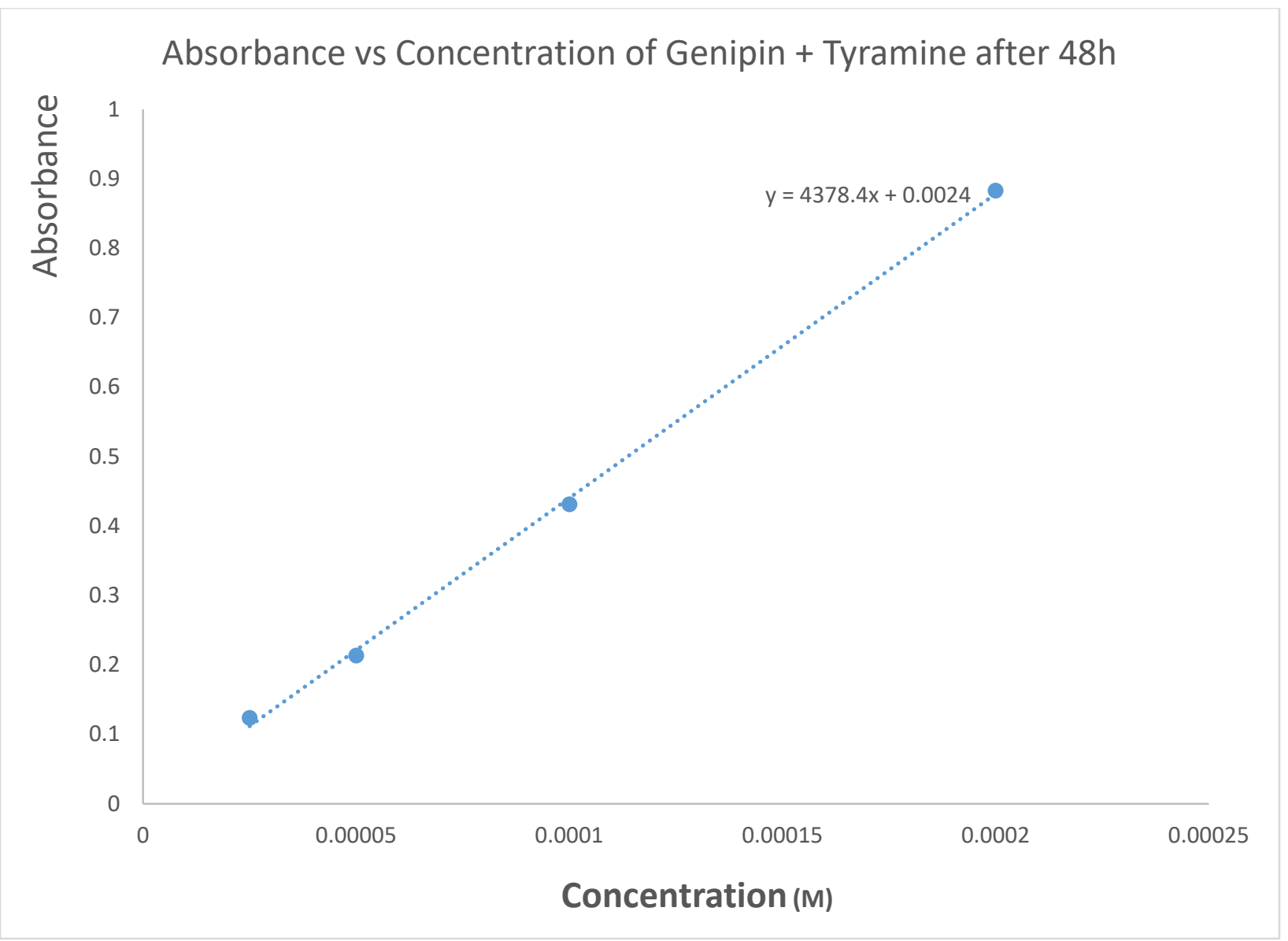

Figure S21 Plot of absorbance versus molar concentration of genipin/tyramine solutions at 48h Molar Absorptivity:

$\varepsilon=\mathrm{A} / \mathrm{cl} \mathrm{I}=1.00 \mathrm{~cm} ; \mathrm{A} / \mathrm{c}=4378.4 \mathrm{~L} / \mathrm{mol}$ thus $\varepsilon=4.4 \times 10^{3} \mathrm{~L} / \mathrm{mol} \mathrm{cm}$ 


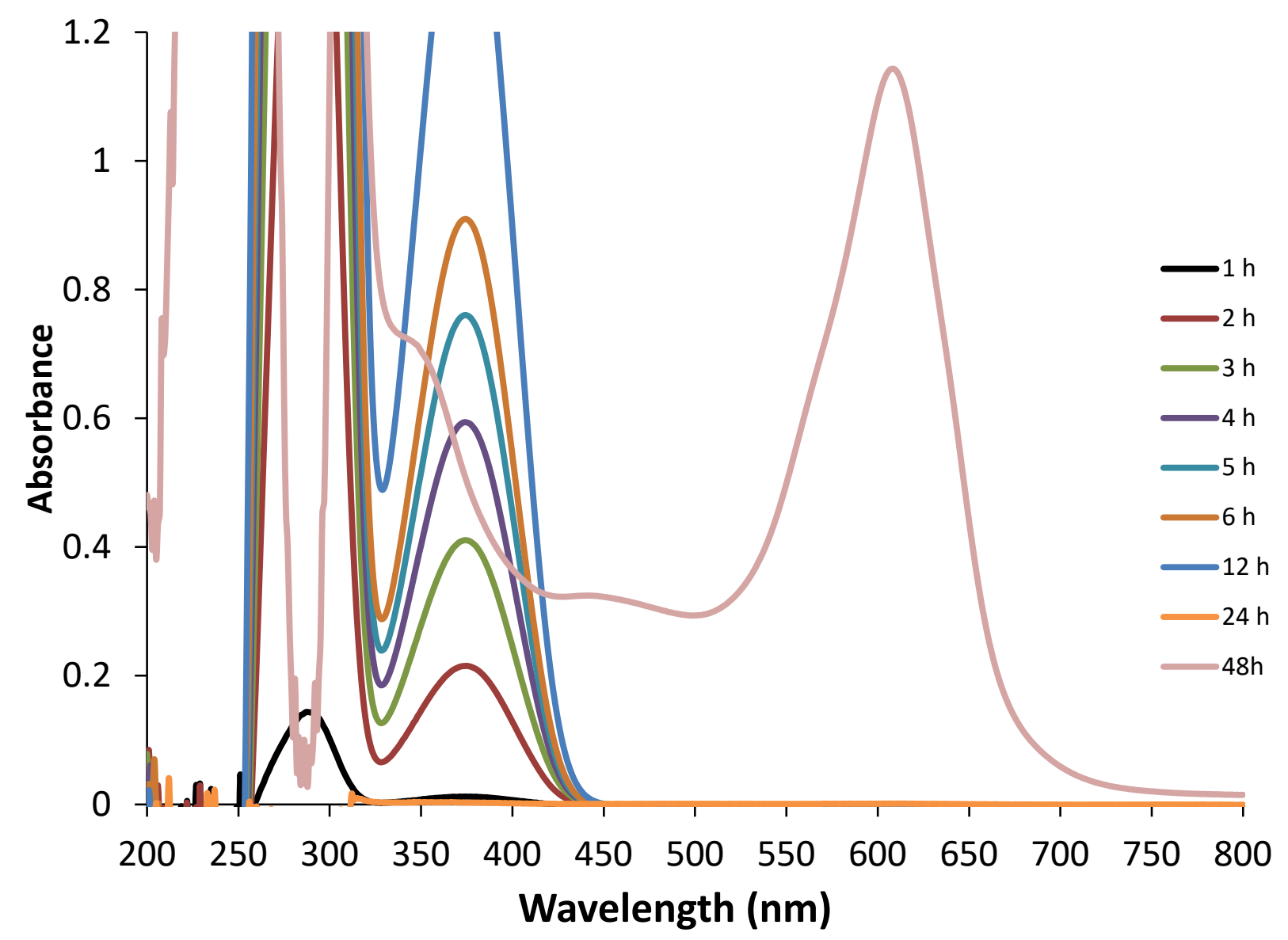

Figure S22 - UV-visible absorbance spectra of $\mathrm{MeOH}$ solutions $2.0 \times 10^{-3} \mathrm{M}$ in both histamine and genipin over 1-48h. 


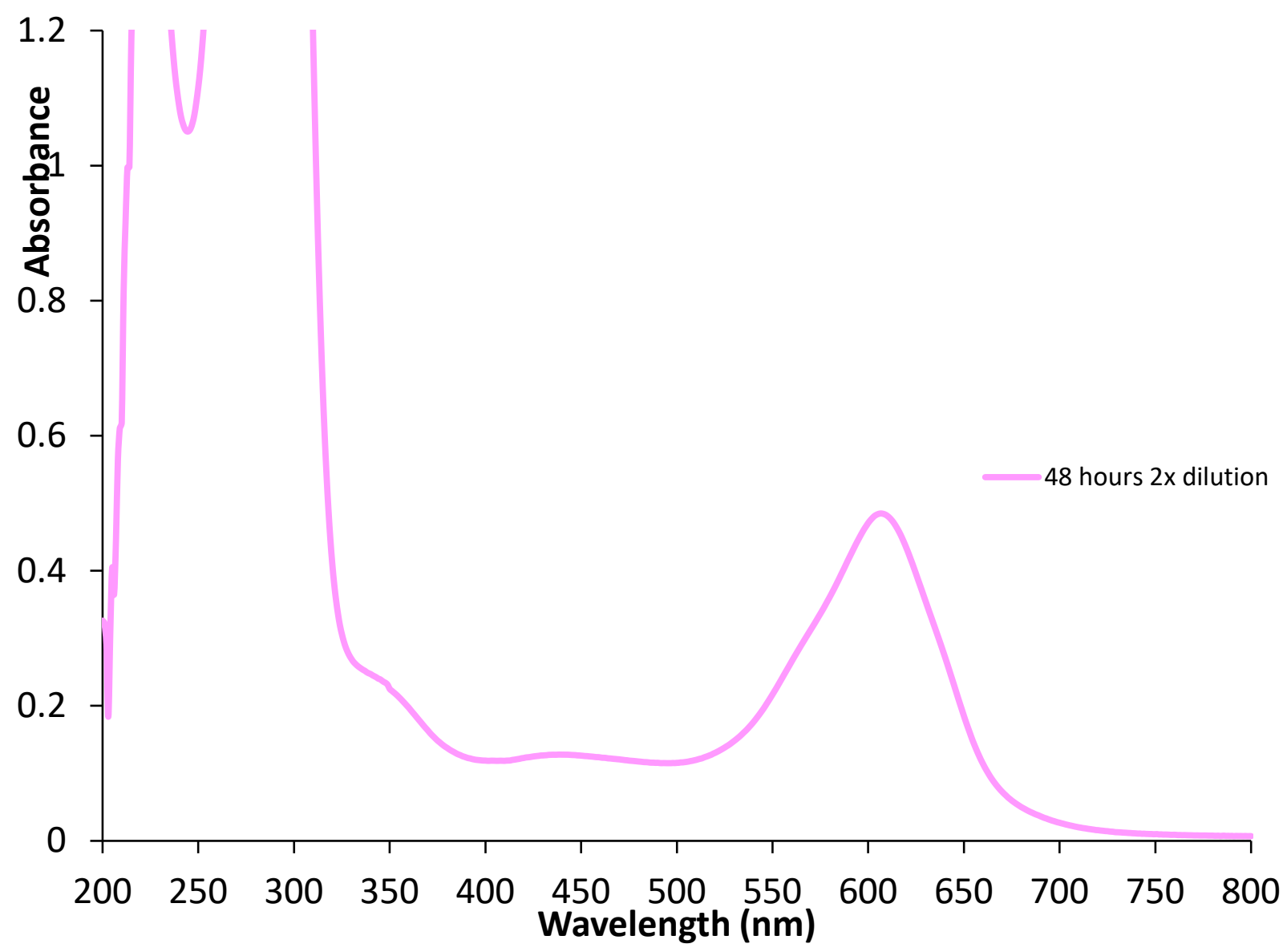

Figure S23 - UV-visible absorbance spectra of $\mathrm{MeOH}$ solution $2.0 \times 10^{-3} \mathrm{M}$ in both histamine and genipin after $48 \mathrm{~h}$ and diluted $2 \mathrm{X}$ at time of measurement. 


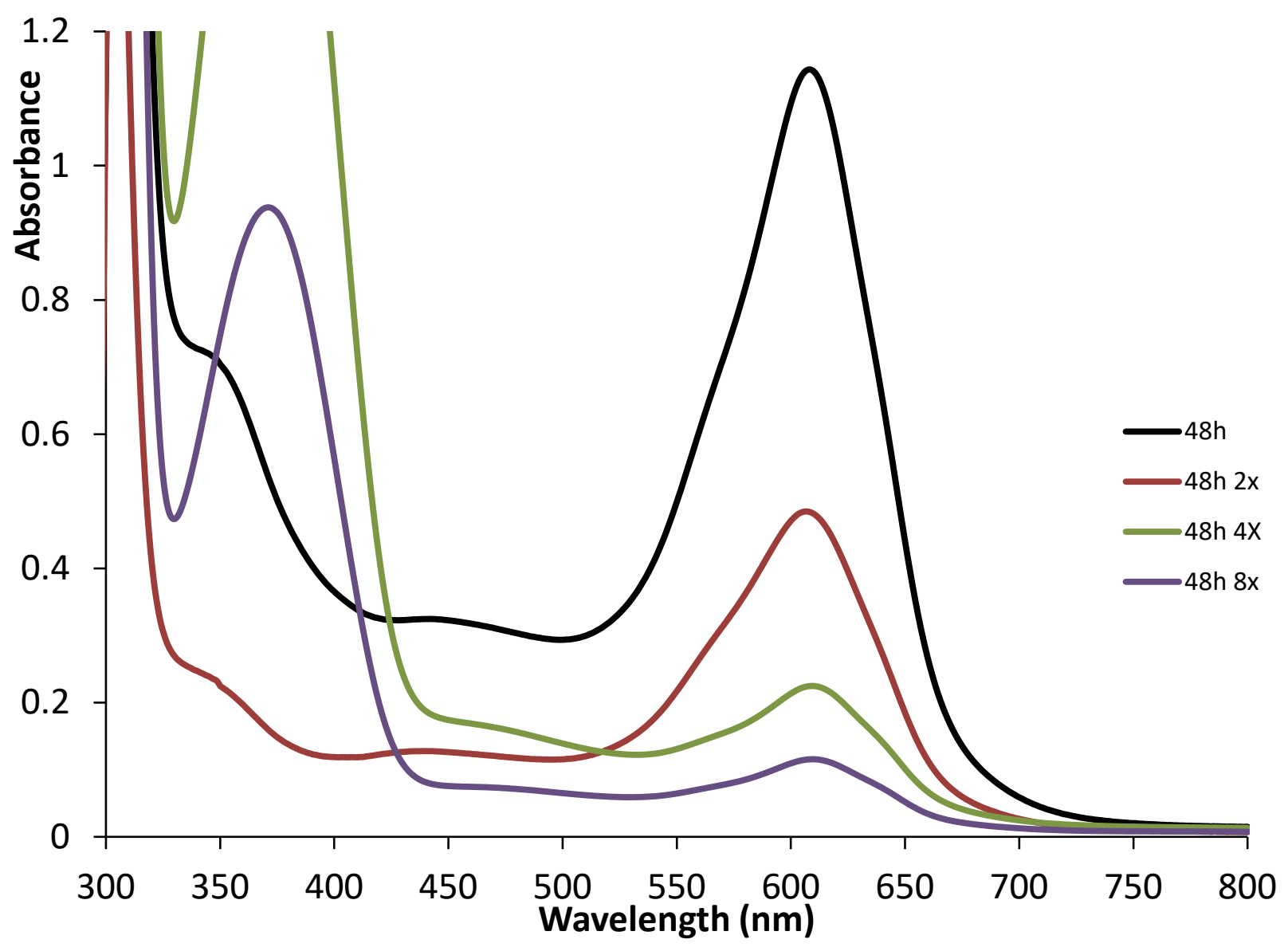

Figure S24 - UV-visible absorbance spectra of $\mathrm{MeOH}$ solutions $2.0 \times 10^{-3} \mathrm{M}$ in both genipin and histamine $2.0 \times 10^{-3} \mathrm{M}$ over $1-48 \mathrm{~h}$ at various dilutions 


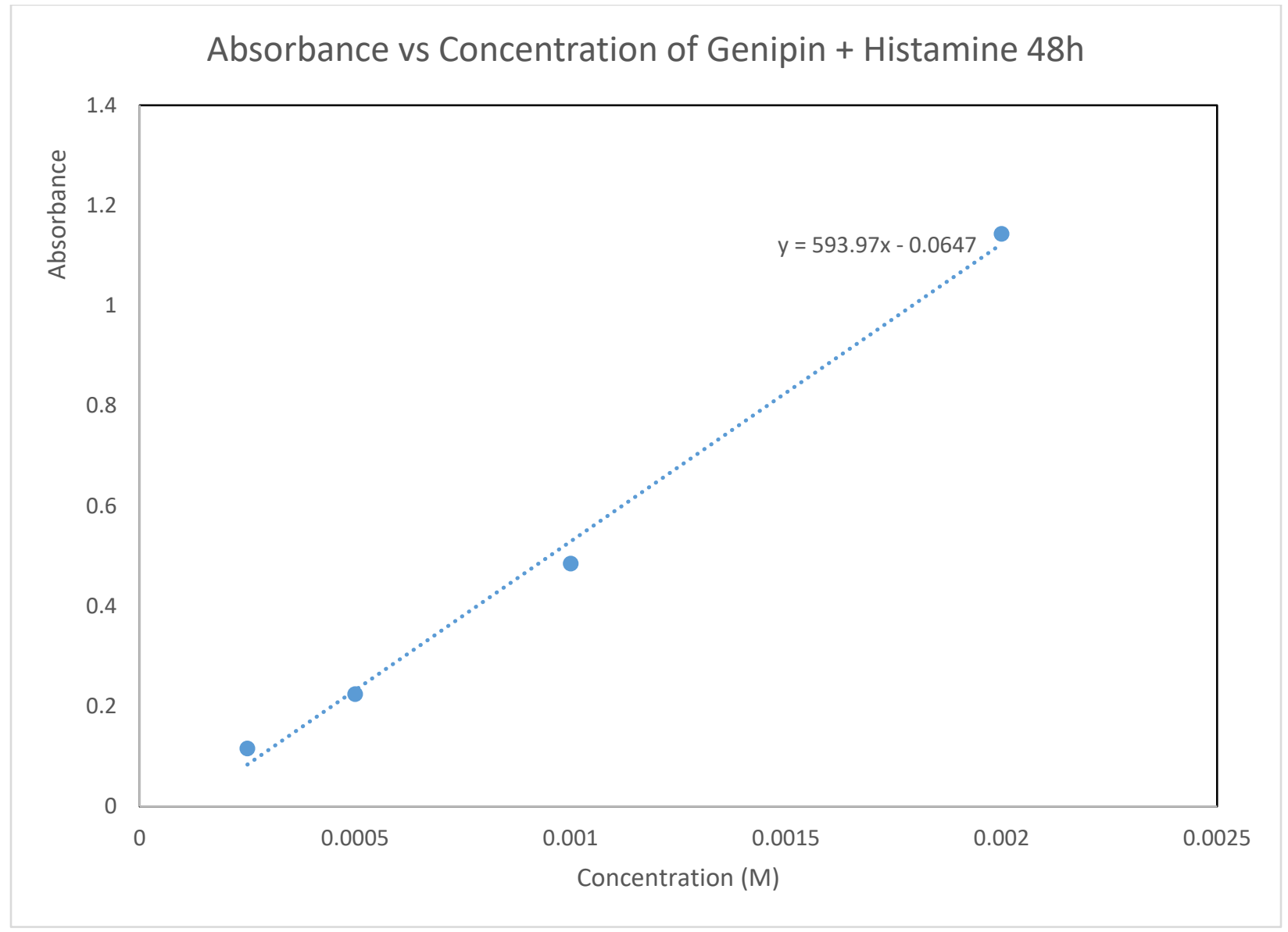

Figure S25 Plot of absorbance versus molar concentration of genipin/histamine solutions at $48 \mathrm{~h}$ Molar Absorptivity:

$\varepsilon=\mathrm{A} / \mathrm{cl} \mathrm{I}=1.00 \mathrm{~cm} ; \mathrm{A} / \mathrm{c}=593.97 \mathrm{~L} / \mathrm{mol}$ thus $\varepsilon=6.0 \times 10^{2} \mathrm{~L} / \mathrm{mol} \mathrm{cm}$ 


\subsection{Estimating the Visual Detection Limit of Amines with a 0.500 M Solution of Genipin}

$20.0 \mathrm{~mL}$ of $0.500 \mathrm{M}$ methanolic solution of genipin was prepared by dissolving $2.26 \mathrm{~g}(0.0100$ $\mathrm{mmol}$ ) genipin in a $50: 50$ mixture of $10.0 \mathrm{~mL}$ methanol and $10.0 \mathrm{~mL}$ water in a $50 \mathrm{~mL}$ round bottom flask (50:50 water:methanol demonstrated experimentally greater genipin solubility than $100 \%$ methanol or $100 \%$ water)

Of the UV-visible absorbance spectra of 1:1 solutions of genipin with biogenic amines putrescine, cadaverine, tyramine, and histamine, the blue dye formed by the reaction of genipin with tyramine exhibited the largest molar absorptivity, at $4.4 \times 10^{3} \mathrm{~L} / \mathrm{mol} \mathrm{cm}$. Thus, visual detection of tyramine should be observable at the lowest possible concentration.

A $0.100 \mathrm{M}$ solution of tyramine in methanol was prepared by dissolving $137 \mathrm{mg}(1.00 \mathrm{mmol})$ tyramine in $10.0 \mathrm{~mL}$ methanol. A stock solution with a concentration of $1.0 \times 10^{-3} \mathrm{M}$ was prepared by diluting $20 \mu \mathrm{L}$ of the $0.100 \mathrm{M}$ solution to a total volume of $2.0 \mathrm{~mL}$ with methanol.

$35 \mu \mathrm{L}$ of the stock solution of tyramine concentration $1.0 \times 10^{-3} \mathrm{M}$ was then added to $3.5 \mathrm{~mL}$ of the $0.500 \mathrm{M}$ solution of genipin to produce a solution $1.0 \times 10^{-5} \mathrm{M}(1.4 \mathrm{ppm})$ in tyramine (Solution 1).

$165 \mu \mathrm{L}$ of the stock solution of tyramine concentration $1.0 \times 10^{-3} \mathrm{M}$ was then added to $3.3 \mathrm{~mL}$ of the $0.500 \mathrm{M}$ solution of genipin to produce a solution $5.0 \times 10^{-5} \mathrm{M}(6.9 \mathrm{ppm})$ in tyramine (Solution 2).

Solutions were sealed in 1-dram vials to minimize headspace for evaporation and left for 48 hours. After 48 hours, Solution 1 appeared identical to the naked eye to a solution containing only $0.500 \mathrm{M}$ genipin, while Solution 2 had taken on a greenish colour (Figure S27).

UV-visible absorbance spectrum was obtained for Solution 2 (Figure S26). 


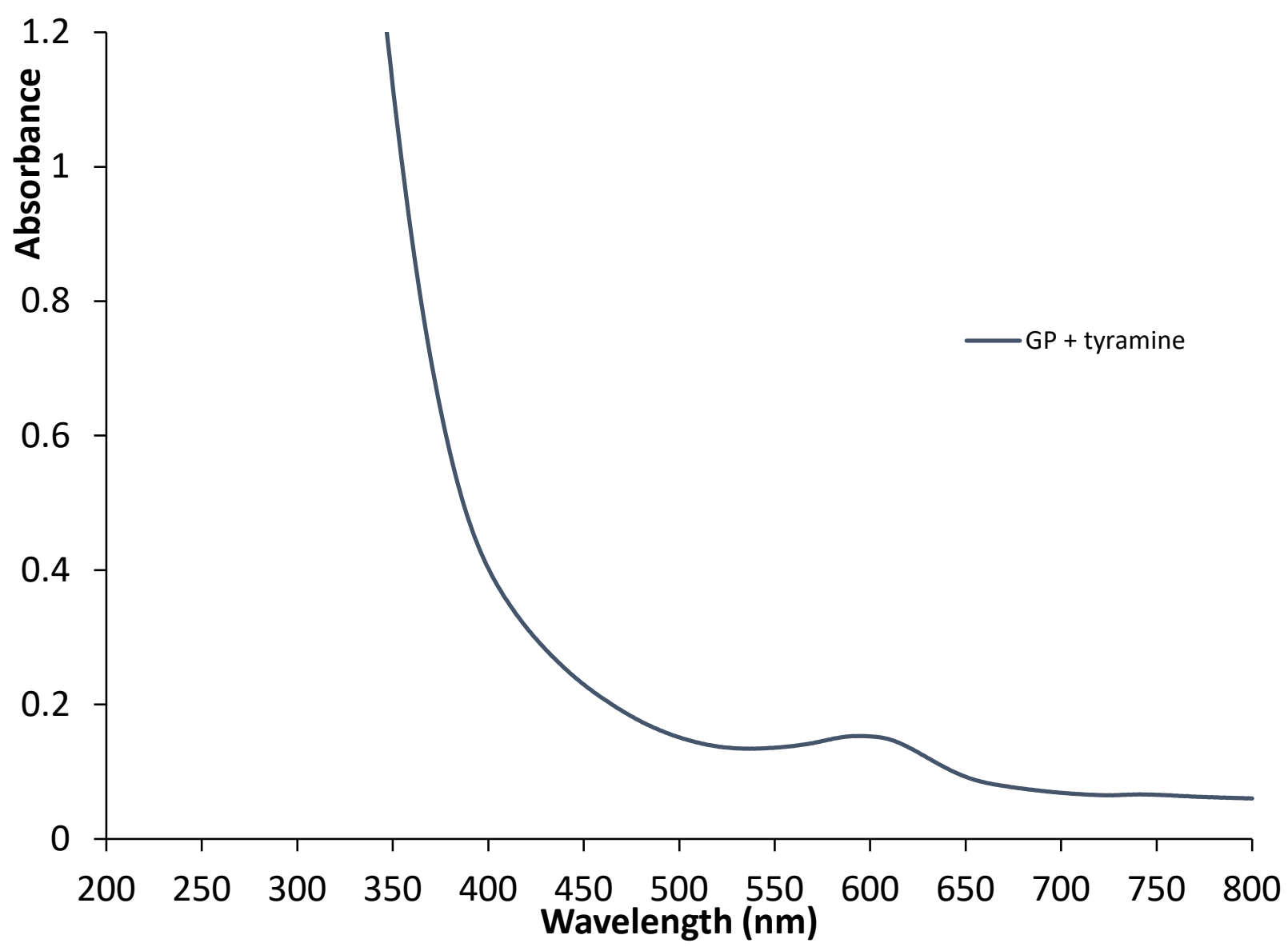

Figure S26 -UV-Visible absorbance spectrum of reaction mixture of tyramine at $5.0 \times 10^{-5} \mathrm{M}$ concentration and genipin at $0.50 \mathrm{M}$ concentration in $50: 50 \mathrm{MeOH}: \mathrm{H}_{2} \mathrm{O}$ After $48 \mathrm{~h}$. 


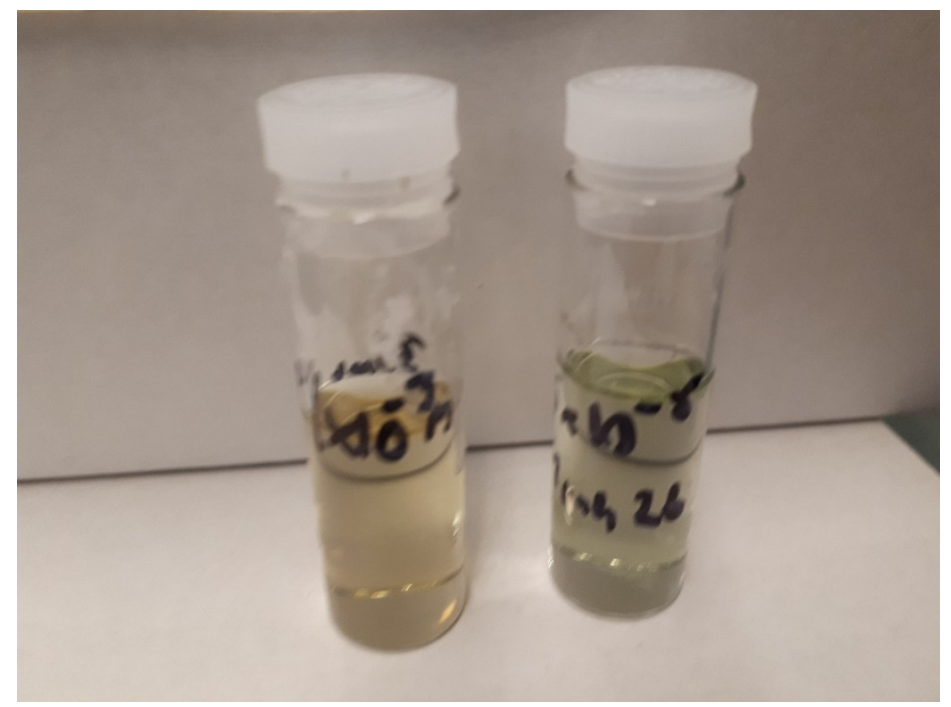

Figure S27: $0.50 \mathrm{M}$ solutions of genipin in 50:50 water/methanol spiked with $1.4 \mathrm{ppm}$ tyramine (left) and 6.9 ppm tyramine (right) 


\section{Colorimetric Response of Genipin-Infused Gel Beads Exposed to Amine Vapor}

\section{Preparation of Genipin-Impregnated Calcium Alginate Gel Beads}

$1,10,50$, or $100 \mathrm{mg}$ of genipin was dissolved in $10.0 \mathrm{~mL}$ of distilled water in a $100 \mathrm{~mL}$ beaker. In the case of the addition of $100 \mathrm{mg}$ of genipin, heating to $80^{\circ} \mathrm{C}$ was required for full dissolution. $200 \mathrm{mg}$ of sodium alginate powder was added with vigorous stirring to form the sodium alginate gel. Using a variation of the simple dripping (extrusion) technique described in the review by Bhandari et. al. (see reference 20 in the main manuscript) a $1 \mathrm{~mL}$ disposable syringe without a needle was employed to uptake $1.00 \mathrm{~mL}$ of sodium alginate gel. Due to dripping of gel from the outside of the syringe, the first 1-2 drops were dispensed back into the gel mixture. The remainder was dispensed dropwise from a height of $10 \mathrm{~cm}$ above the surface of $25.0 \mathrm{~mL}$ of a 1.0 $\% \mathrm{w} / \mathrm{v} \mathrm{CaCl}$ solution contained in a $125 \mathrm{~mL}$ Erlenmeyer flask. $12-15$ beads were dispensed in this manner until the syringe contained $>0.1 \mathrm{~mL}$ of gel, at which point the syringe was re-filled to avoid size decrease of gel beads due to air bubbles inside the syringe and lack of material. After 100-110 beads had been dispensed in this manner, using $75-80 \%$ of the sodium alginate gel mass, the remaining $\mathrm{CaCl}_{2}$ solution was decanted from the beads and the beads were washed with 10 $\mathrm{mL}$ of an aqueous solution of $0.1 \mathrm{M} \mathrm{CH}{ }_{3} \mathrm{COOH} / \mathrm{CH}_{3} \mathrm{COONa}$ buffer. Beads were then dispensed onto a watch glass, separated using tweezers and let stand 1 hour to allow excess water to run onto the glass. The beads were then removed from the watch glass and stored in a sealed vial in a refrigerator at $4^{\circ} \mathrm{C}$.

\subsection{Genipin-Infused Gel Beads Exposed to Putrescine Vapor}

\section{General Procedure}

1-10 genipin-embedded calcium alginate gel beads were placed in a $20 \mathrm{~mL}$ vial using a clean pair of tweezers. $0.10 \mathrm{mmol}$ of primary amine was added to a $1 \mathrm{dram}$ vial (or Eppendorf tube) which was placed, uncapped, inside the $20 \mathrm{~mL}$ vial containing the beads, and the larger vial was sealed with a cap and either left at room temperature or placed in a refrigerator at $4{ }^{\circ} \mathrm{C}$ and monitored visually for up to 48 hours.

In the case where 10 gel beads were exposed to $0.10 \mathrm{mmol}$ amine, after 24 hours at room temperature the gel beads remained colourless, while after 48 hours all ten beads had turned blue. In the case where only one bead was used instead of ten beads, the single bead turned deep blue within twenty-four hours. 
Dissolution of gel beads to obtain UV spectra after specified reaction times of 6-48 hours, 10 dyed genipin-containing gel beads were dissolved in $3.5 \mathrm{~mL}$ of a $55 \mathrm{mM}$ solution of sodium citrate to allow dissolution of gel beads by to cation exchange mediated by the citrate cation chelator. ${ }^{21}$

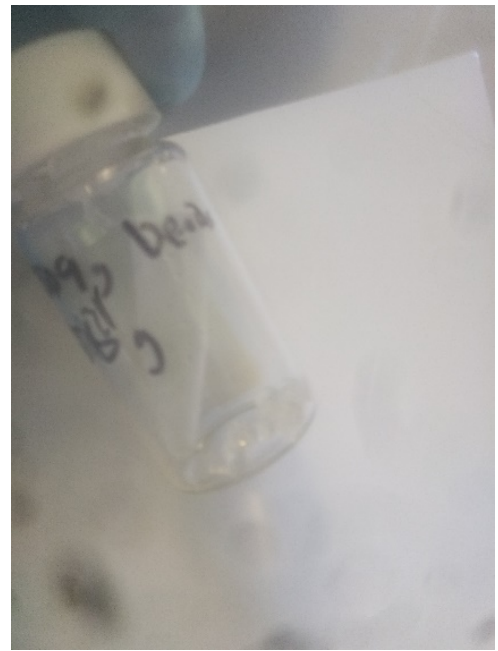

A

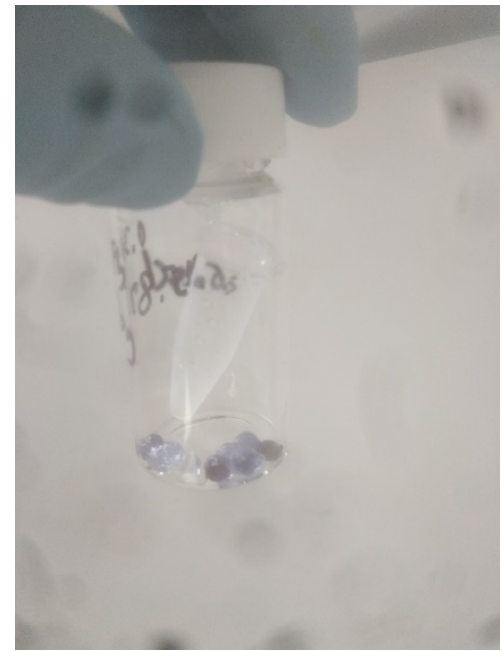

B

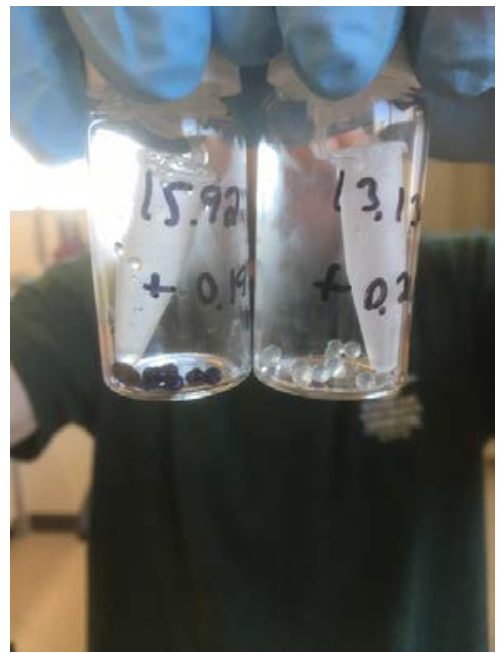

C

Figure S28: A) gel beads at $t=0$ with putrescine beaker B) gel beads after $24 \mathrm{~h}$ reaction with putrescine vapour C) comparison of gel beads in reaction with putrescine vapour after $48 \mathrm{~h}$ at room temperature (left vial) and in the refrigerator (right vial) 


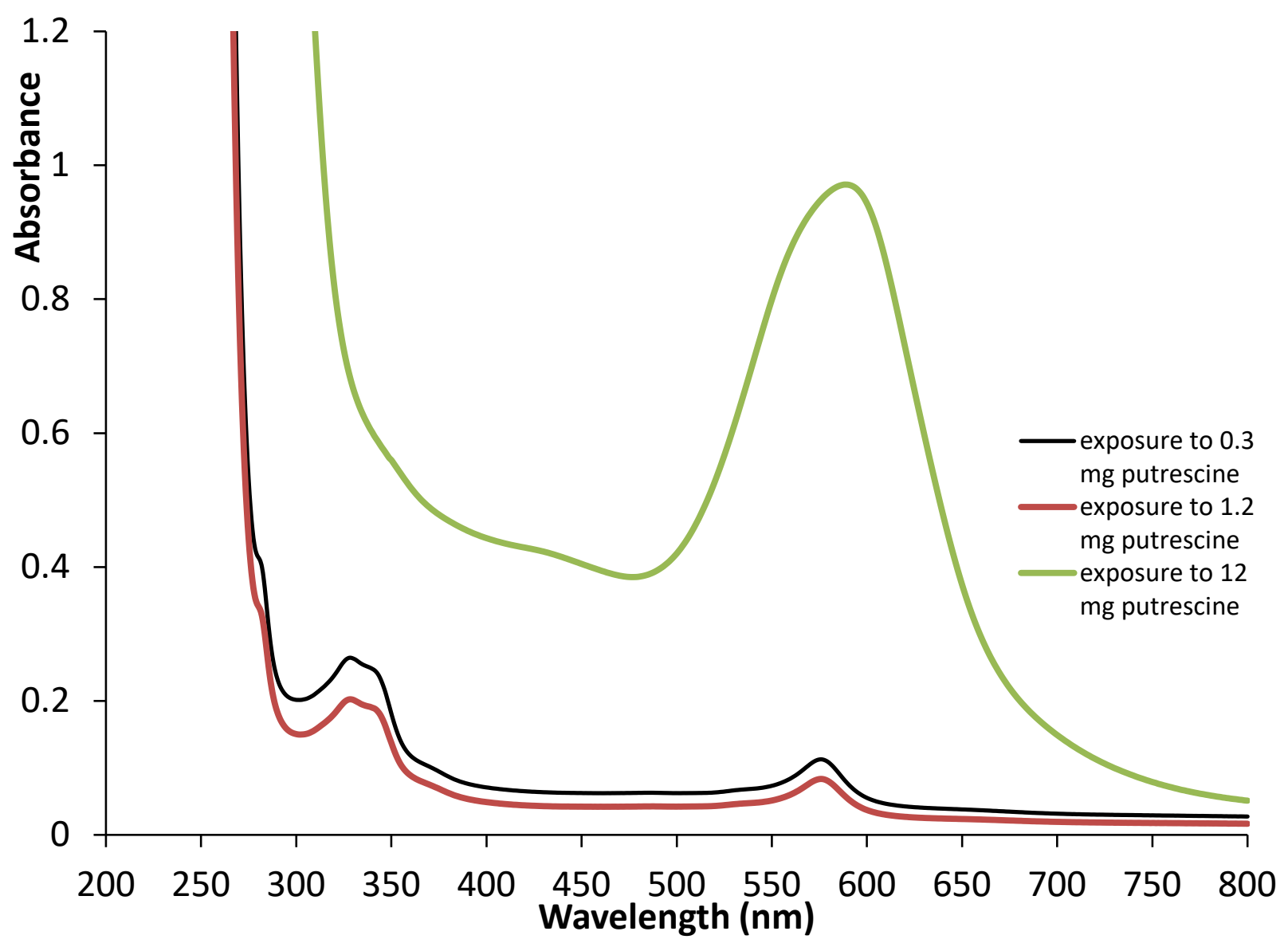

Figure S29: UV-visible absorbance spectra of dissolved genipin-infused gel beads after exposure to $0.3 \mathrm{mg}, 1.2 \mathrm{mg}$, or $12 \mathrm{mg}$ of putrescine. 


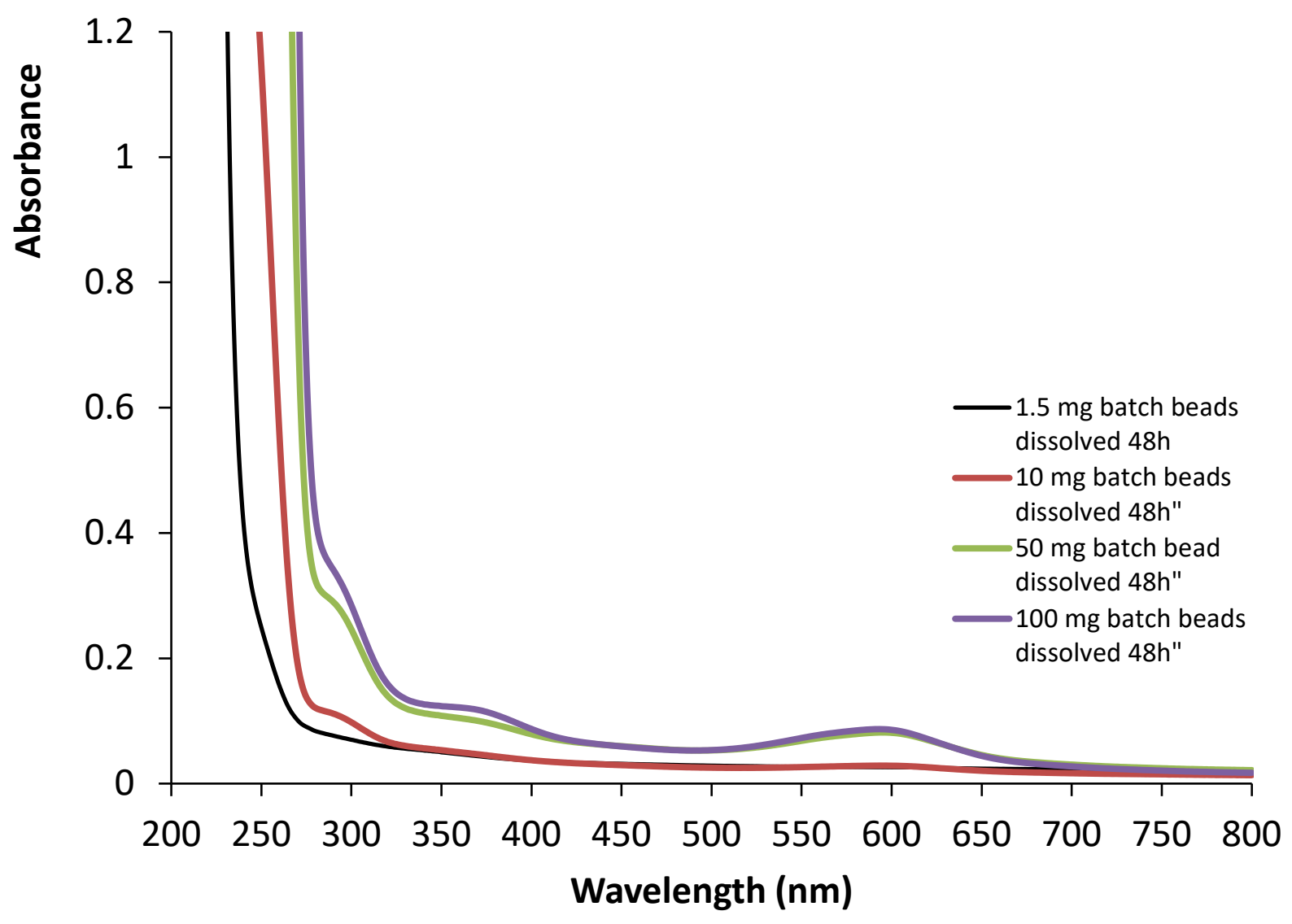

Figure S30: UV-visible absorbance spectra of dissolved gel beads containing 4 different concentrations of genipin, after exposure to $0.10 \mathrm{mmol}(8.8 \mathrm{mg})$ putrescine for $48 \mathrm{~h}$ 


\section{Colorimetric Response of Genipin-Infused Gel Beads Exposed to Raw Chicken}

Table S1 - Genipin-infused calcium alginate gel beads exposed to freshly purchased raw chicken at room temperature (left) and refrigerated at $4{ }^{\circ} \mathrm{C}$ (right)

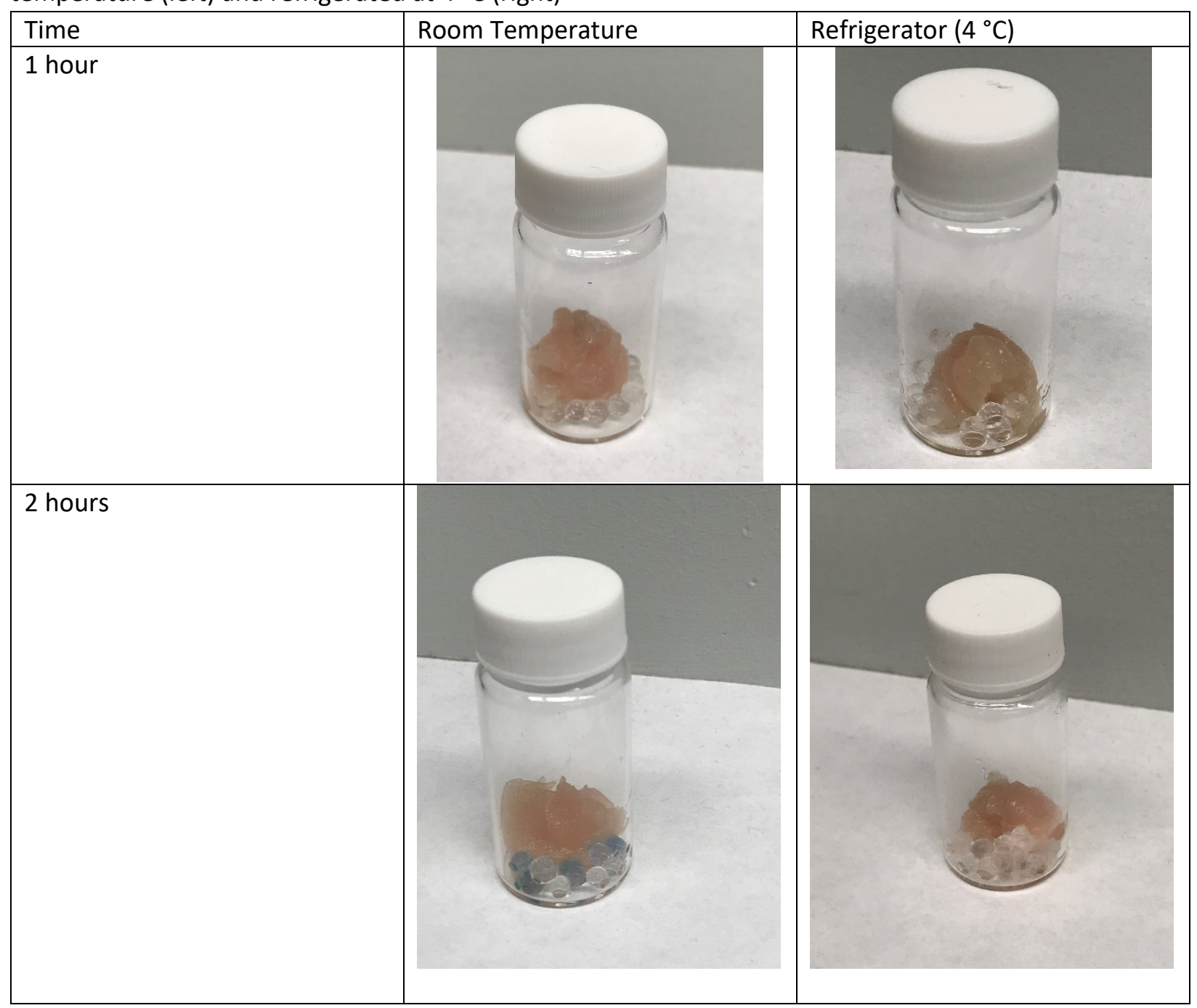




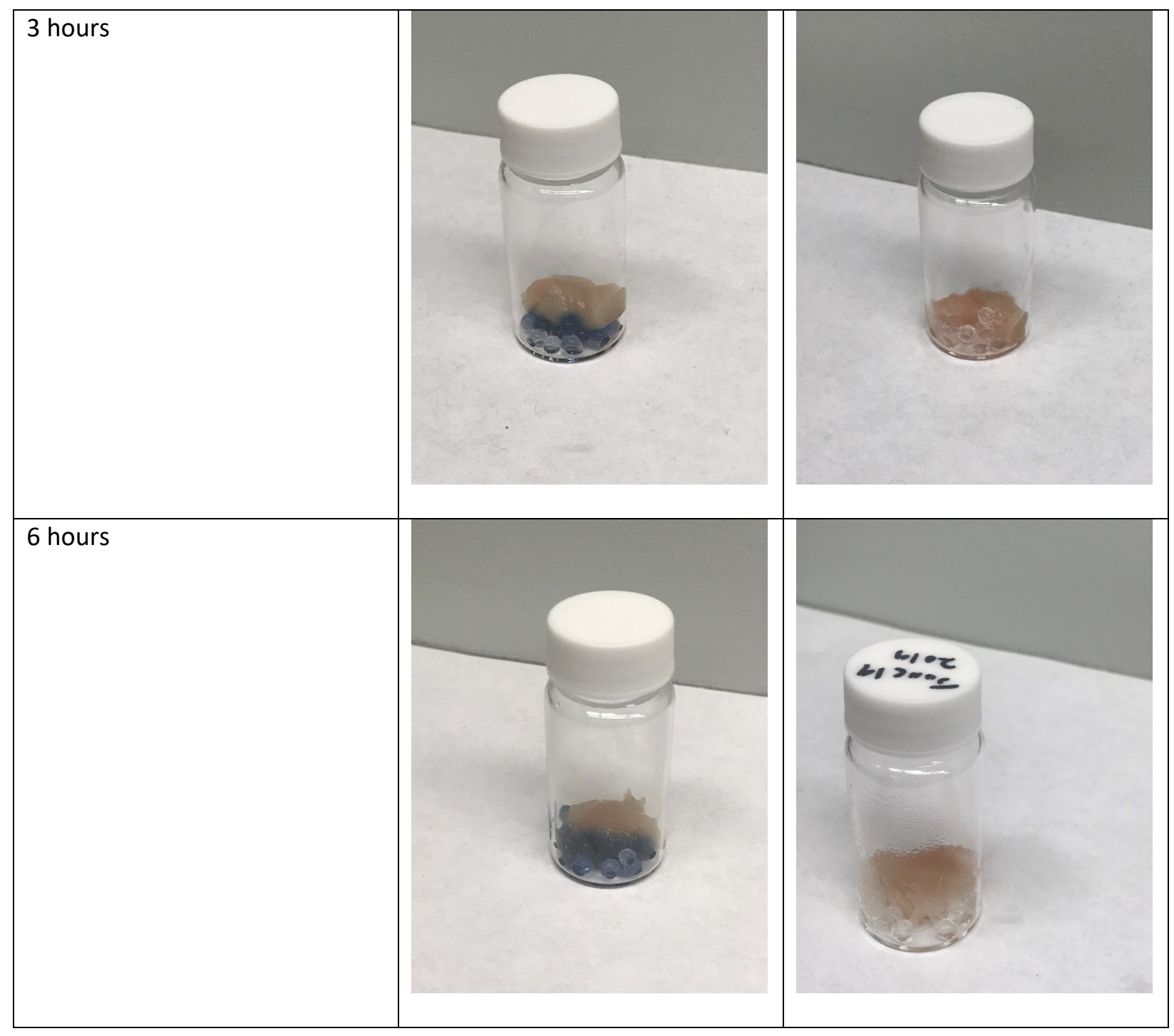

\section{Quantification of color from images}

The software Adobe Photoshop was used to quantify colors in images using International Commission on Illumination (CIE) guidelines with chromatic values $L^{*} a^{*} b^{*}$ representing lightness $\left(L^{*}\right)$ and color opponent red/green $\left(a^{*}\right)$ and yellow/blue $\left(b^{*}\right)$ dimensions. We followed the procedure outlined by Read de Alaniz ${ }^{1}$ where the Delta $\mathrm{E}\left(\Delta \mathrm{E}^{*}\right)$ value (the distance between two chromatic values in color space) is used as a metric to differentiate two colors. ${ }^{1}$

\footnotetext{
${ }^{1}$ Chem. Eur. J. 2017, 23, 3562-3566; J. Food Eng. 2004, 61, 137-142; Measuring Color: Fourth Edition, 2011; Food Res. Int. 2006, 39, 1092-1098.
} 


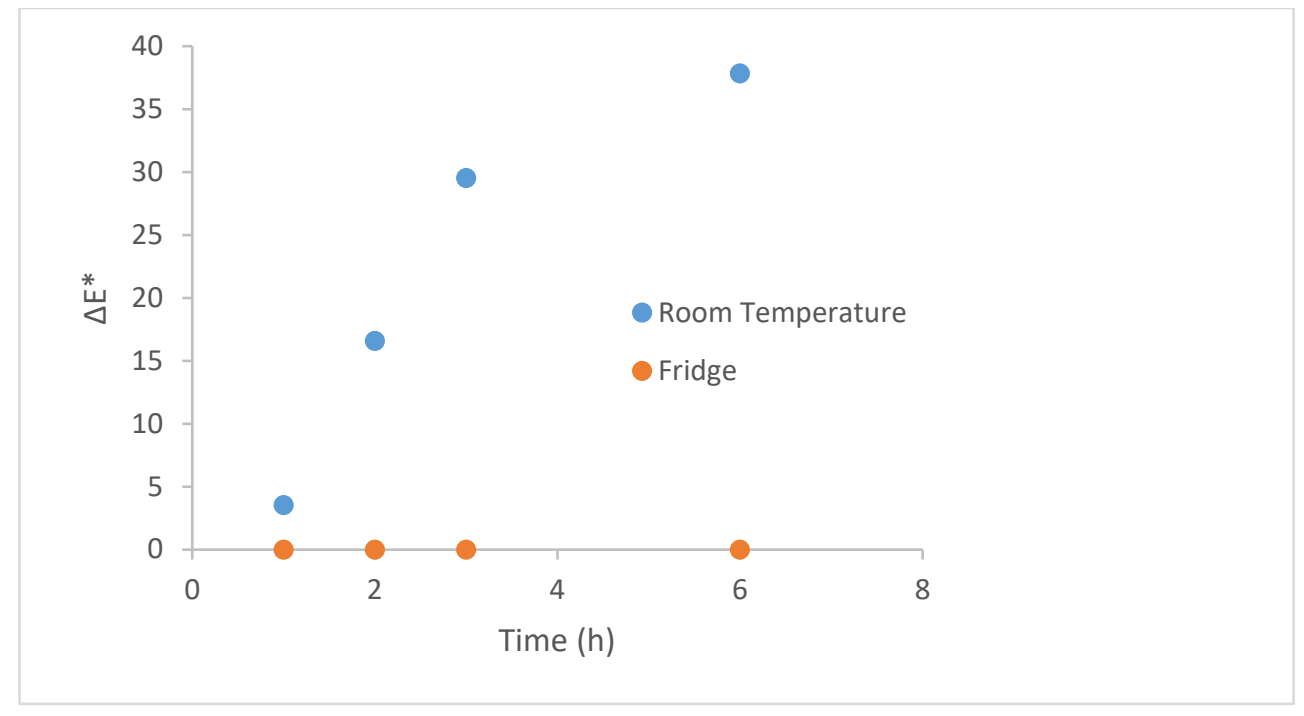

Figure S31: Change in color as measured by $\Delta \mathrm{E}^{*}$ of the beads at room temperature relative to the beads in the fridge. 
General Procedure for Reaction of Genipin with Benzylamine, N-methylbenzylamine, and N,Ndimethylbenzylamine

To a solution of $23 \mathrm{mg}(0.10 \mathrm{mmol})$ genipin in $1 \mathrm{~mL} \mathrm{CD} \mathrm{CD}_{3} \mathrm{O}$ in a 4 dram vial was added a solution of 0.10 mmol of the respective amine. A small stir bar was added and solutions were allowed to stir for $24 \mathrm{~h}$. An aliquot of the resulting blue solution was then decanted into an NMR tube and ${ }^{1} \mathrm{H}$ NMR spectrum was obtained.

${ }^{1} \mathrm{H}$ NMR spectrum of genipin in $\mathrm{CD}_{3} \mathrm{OD}$

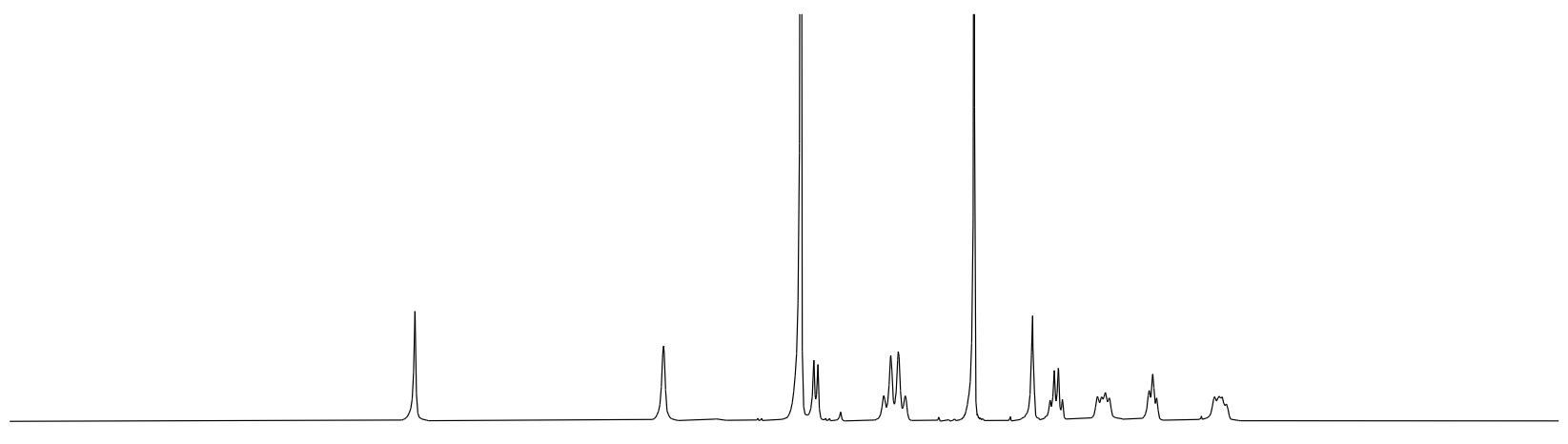


${ }^{1} \mathrm{H}$ NMR spectrum of benzylamine in $\mathrm{CD}_{3} \mathrm{OD}$

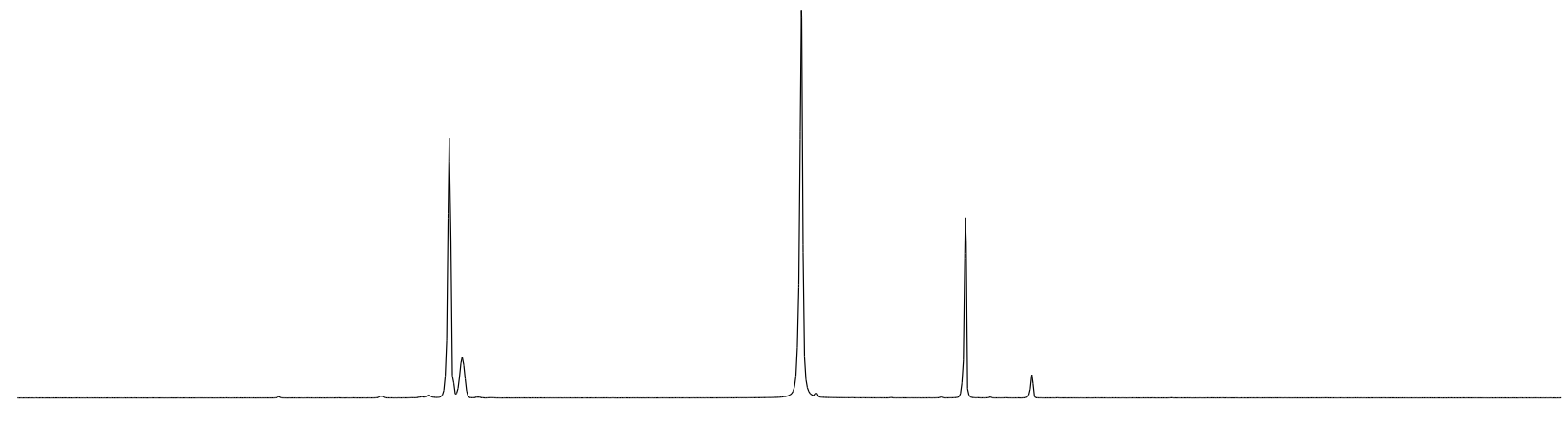

${ }^{1} \mathrm{H}$ NMR spectrum of reaction mixture of benzylamine and genipin in $\mathrm{CD}_{3} \mathrm{OD}$

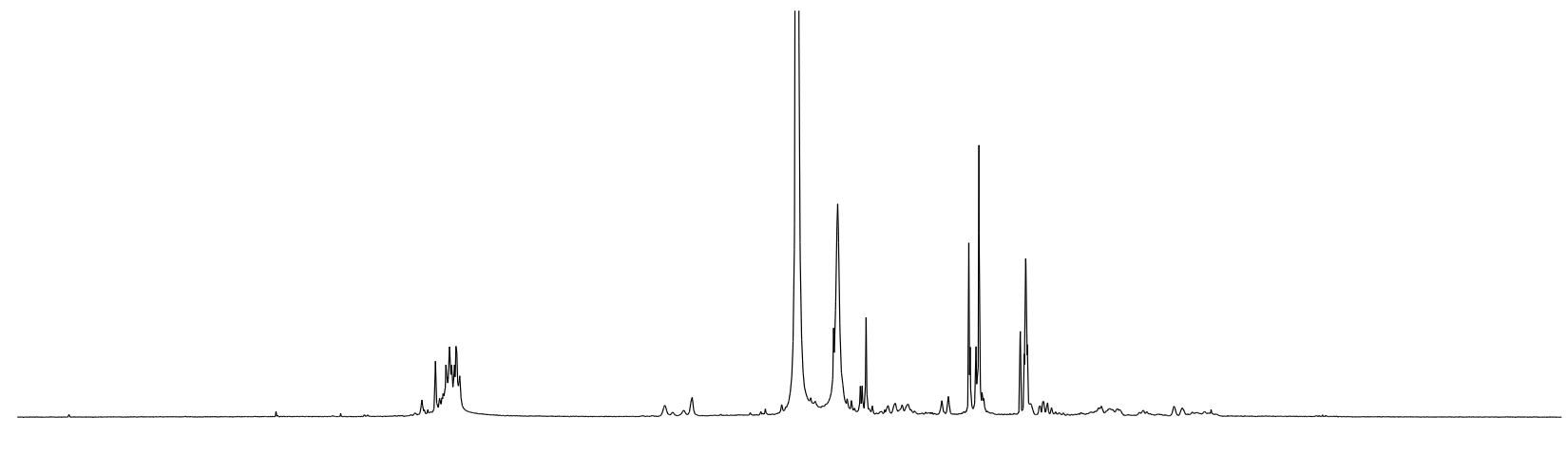


${ }^{1} \mathrm{H}$ NMR spectrum of $\mathrm{N}$-methylbenzylamine in $\mathrm{CD}_{3} \mathrm{OD}$

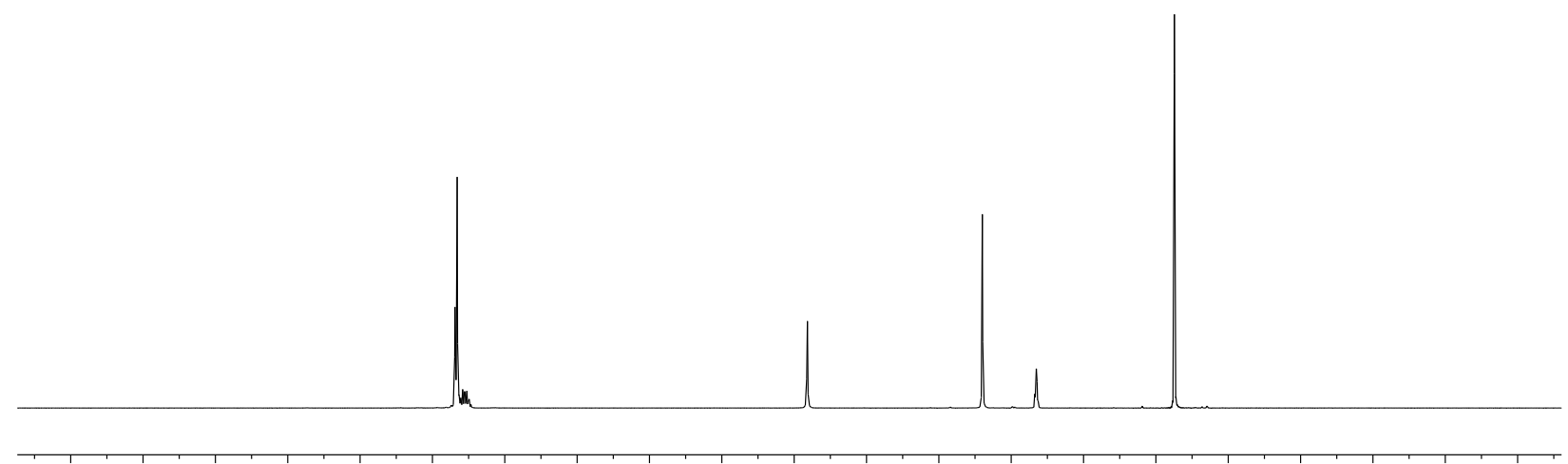

${ }^{1} \mathrm{H}$ NMR spectrum of reaction mixture of $\mathrm{N}$-methylbenzylamine and genipin in $\mathrm{CD}_{3} \mathrm{OD}$

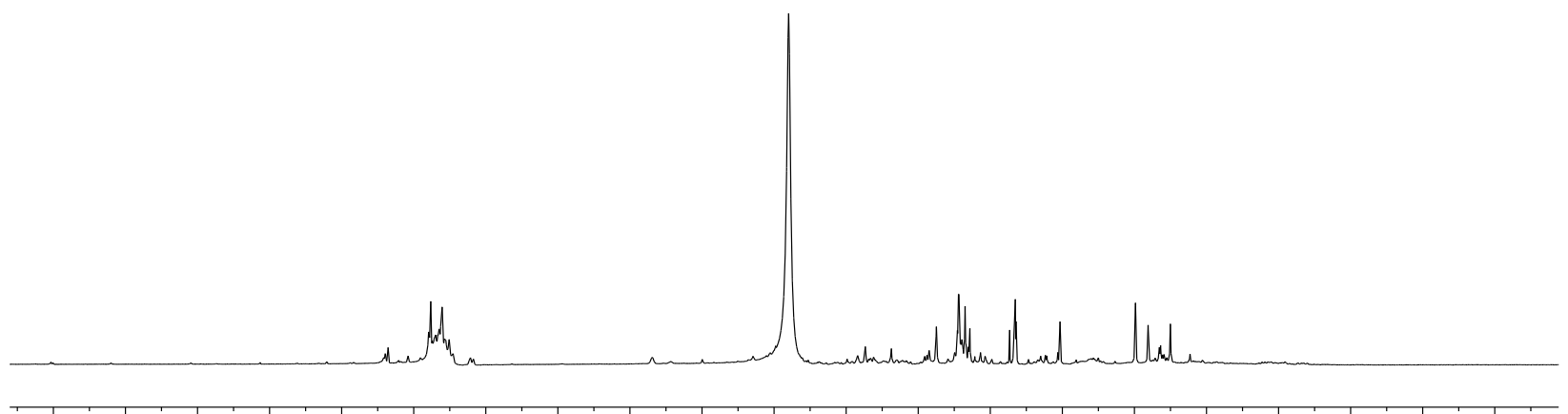


${ }^{1} \mathrm{H}$ NMR spectrum of $\mathrm{N}, \mathrm{N}$-dimethylbenzylamine in $\mathrm{CD}_{3} \mathrm{OD}$

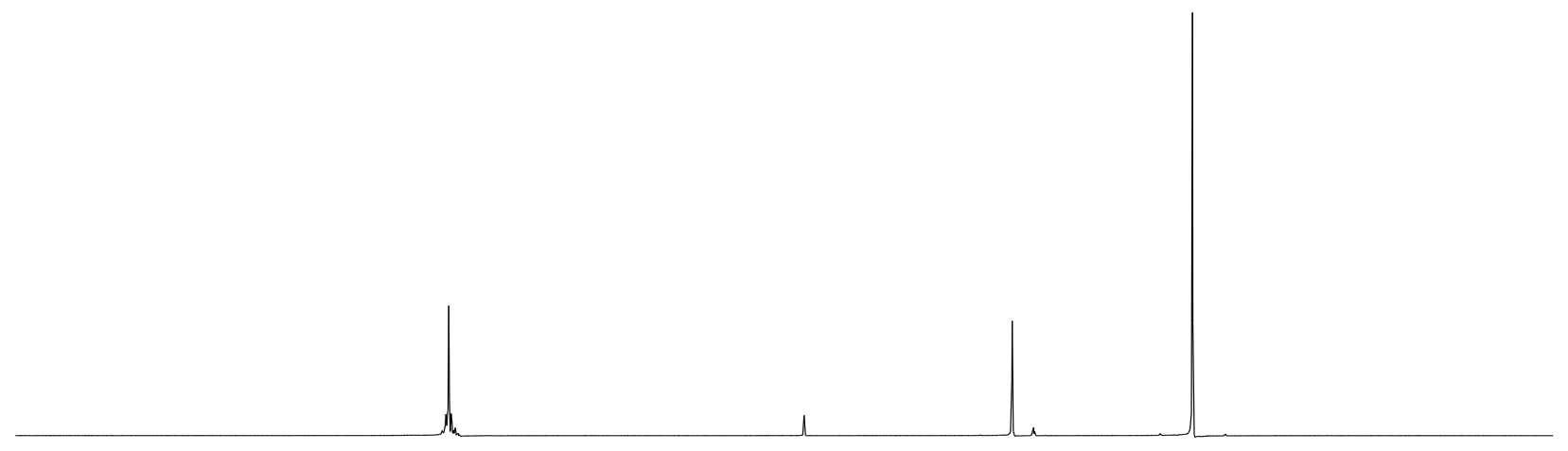

${ }^{1} \mathrm{H}$ NMR spectrum of reaction mixture of $\mathrm{N}, \mathrm{N}$-dimethylbenzylamine and genipin in $\mathrm{CD}_{3} \mathrm{OD}$

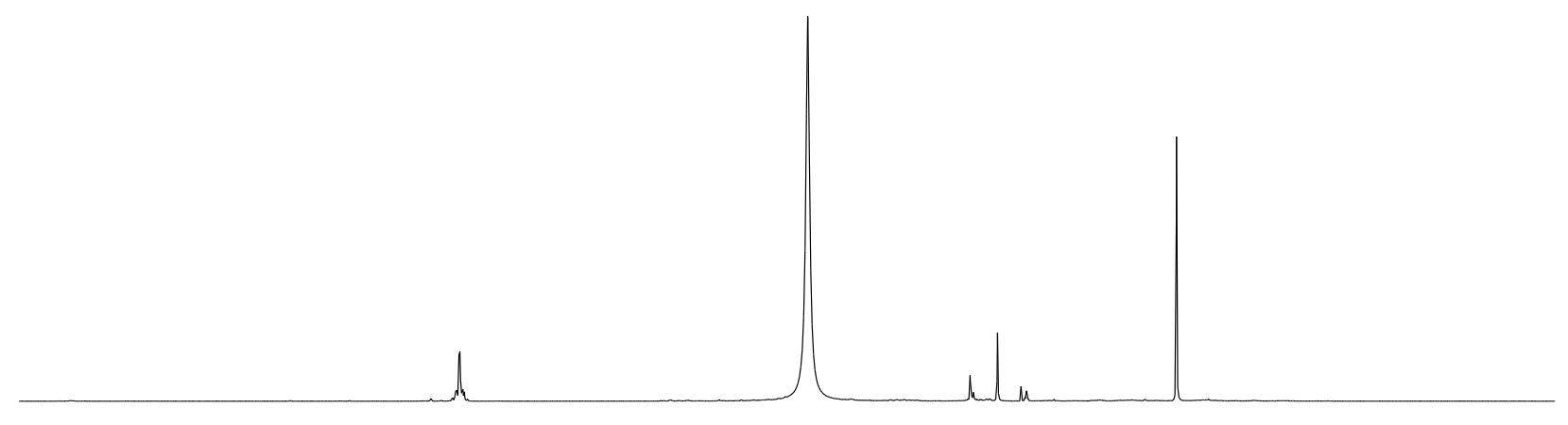




\title{
A Dual Sensor for Biogenic Amines and Oxygen Based on Genipin Immobilized in Edible Calcium Alginate Gel Beads
}

\author{
Ian Mallov, Fiona Jeeva and Christopher B. Caputo* \\ Department of Chemistry, York University, Toronto, ON, M3J 1P3, Canada
}

\begin{abstract}
Food is often wasted due to real or perceived concerns about preservation and shelf life. Thus, precise, accurate and consumer-friendly methods of indicating whether food is safe for consumers are drawing great interest. The colorimetric sensing of biogenic amines released as food degrades is a potential way of determining the quality of the food. Herein, we report the use of genipin, a naturally occurring iridoid, as a dual colorimetric sensor for both oxygen and biogenic amines. Immobilization of genipin in edible calcium alginate beads demonstrates that it is a capable sensor for amine vapors and can be immobilized in a non-toxic, food-friendly matrix.
\end{abstract}

\section{Introduction}

Biogenic amines (BAs) are a major indicator of food spoilage, particularly in meat and fish, but also in such food and drink as beer, red wine, mushrooms, and aged cheese. ${ }^{1}$ BAs are produced when food is exposed to oxygen gas, allowing for microbial oxidative hydrodecarboxylation of amino acids. They are responsible for the distinctive odor of rotting meat, although adverse effects to human health can occur in concentrations lower than the odor threshold. ${ }^{2-4}$ Globally, nearly $1 / 3$ of all food is wasted, thus precise, accurate and consumer-friendly methods of indicating whether food is safe to consumers are drawing great interest. ${ }^{5}$

A number of instrumental methods and techniques to detect BA's have been developed which utilize conventional chromatography methods, ${ }^{2}$ chemoresistivity, ${ }^{4,6}$ or biological sensing. ${ }^{7}$ However, these methods have limitations for practical use in detecting food spoilage, as the analyses require large or specialized equipment, and these impede their application by the public. Progress has been made on this front and an accessible electrical sensor was recently reported utilizing printed electronic gas sensors onto cellulose fibers to detect ammonia. ${ }^{8}$ On the other hand, visual detection sensors have garnered attention as reliable systems which do not require significant instrumentation. Visual sensors can be subdivided into fluorescent or colorimetric sensors. Both benefit from enhanced sensitivity, ease of use and real-time analysis. However, colorimetric sensors have the advantage of being detectable by the naked eye. Considerable research has been undertaken to develop colorimetric sensors for BAs. Some recent highlights of $\mathrm{BA}$ detection include copper-containing complexes which have shown selective detection for spermidine and spermine, ${ }^{9}$ light-activated diarylethene probes, ${ }^{10}$ azabodipy derivatives, ${ }^{11}$ and derivatives of Meldrum's activated furan ${ }^{12,13}$ (Scheme 1). 
Scheme 1 - Recent Examples of Small Molecules for BA Sensing

Previously reported colorimetric BA sensors still have several drawbacks. Many are non-specific for amine substitution (BAs are generally primary amines) or are made of materials which would be incompatible with food (i.e. toxic or non-edible materials). Recent studies have addressed the latter drawback by utilizing the $\mathrm{pH}$ sensitivity of anthocyanins found in cabbage extracts to detect BA. ${ }^{14,15}$ However, these sensors are not specific for primary BA. Most significantly, current colorimetric sensors cannot detect the cause of food spoilage, oxygen gas, so false positives could occur through simple detection of amino acids found in the food. To address this issue, modified atmospheric packaging is gaining in popularity as a way of excluding oxygen from food and extending shelf lives. ${ }^{16}$

An ideal colorimetric sensor for real-time food spoilage would sense gaseous BAs at low concentrations and rapidly undergo a distinct color change at a reasonable reaction rate. Further, this sensor must have the ability to selectively detect the primary amines as well as the source of the degradation, oxygen gas. This ideal sensor would be composed of cheap, lowtoxicity materials derived from edible sources and possess a minimal environmental footprint. We aimed to design a sensor satisfying as many of these criteria as possible.

Genipin, (Scheme 1, bottom) a naturally occurring, colorless iridoid, has found numerous applications in diverse fields, most commonly used as a non-toxic cross-linking reagent for biomaterials. ${ }^{17,18}$ As an edible fruit extract, genipin also has relatively low toxicity; a recent study shows that neither genipin nor the resultant gardenia blue dye exhibit genotoxicity. ${ }^{19}$ This transformation from a colorless iridoid to dye can be attributed to the fact that genipin reacts with amines through a double condensation reaction forming an oxidatively unstable 
dihydropyridine intermediate (Scheme 1, bottom). In the presence of oxygen, this intermediate further undergoes oxidative oligomerization/polymerization resulting in a brilliant blue dye. ${ }^{20}$ However, to the best of our knowledge genipin has yet to find applications as a sensor, despite this unique reactivity positioning it as an ideal candidate for a colorimetric sensor for food degradation.

We sought to study the ability of genipin to react with BAs and confirm the selectivity towards a broad range of amines. Herein, we describe the use of genipin as the first dual colorimetric sensor for both $\mathrm{O}_{2}$ and BAs in solution and provide a proof of concept in an immobilized form.

\section{Experimental Section}

Materials. All chemical reagents or solvents were purchased from Fisher Scientific or Millipore Sigma and used as received unless otherwise indicated. Genipin was kindly donated by Inkbox Ink and purity verified independently by ${ }^{1} \mathrm{H}$ NMR analysis. Food-grade sodium alginate was purchased from BYOB Cocktail Emporium in Toronto and used without purification. NMR spectra were obtained at $25^{\circ} \mathrm{C}$ on either a Bruker $700 \mathrm{MHz}$ Spectrometer, Bruker DRX $600 \mathrm{MHz}$ Spectrometer, Bruker ARX $400 \mathrm{MHz}$ Spectrometer or, Bruker ARX $300 \mathrm{MHz}$ Spectrometer. Chemical shifts are reported relative to $\mathrm{SiMe}_{4}$ and referenced to the residual solvent signal $\left({ }^{1} \mathrm{H}\right.$, $\left.{ }^{13} \mathrm{C}\left\{{ }^{1} \mathrm{H}\right\}\right)$. NMR spectra were analyzed using either TopSpin 4.0.1 or MestReNova 6.0.2-5475 software. Chemical shifts are reported in ppm and coupling constants as scalar values in $\mathrm{Hz}$. The conventional abbreviations were used as follows: s (singlet), d (doublet), t (triplet), q (quartet), $\mathrm{dd}$ (doublet of doublets), $\mathrm{m}$ (multiplet), br (broad). Absorption measurements were recorded with a Cary 5000 UV-vis-NIR Spectrophotometer from Agilent Technologies. Recordings were obtained at $25^{\circ} \mathrm{C}$ and taken with the instrument operating in dual beam mode and referenced to methanol. All absorption experiments were conducted in quartz cuvettes $(1 \mathrm{~cm} \times 1 \mathrm{~cm})$ equipped with a Teflon seal.

UV-visible absorbance detection of biogenic amines in solution $22.6 \mathrm{mg}(0.10 \mathrm{mmol})$ of genipin was dissolved in $45.0 \mathrm{~mL}$ methanol in a single-neck round-bottom flask by stirring for five minutes with a magnetic stirrer. For 1:1 solutions of genipin:BA, $0.10 \mathrm{mmol}$ of BA were added to a separate pre-weighed vial and rinsed into the genipin solution with a further $5.0 \mathrm{~mL}$ methanol to prepare $50.0 \mathrm{~mL}$ of solution of concentrations $2.0 \times 10^{-3} \mathrm{M}$ in genipin and BA. For solutions lower in concentration of $\mathrm{BA}$, stock solutions $0.020 \mathrm{M}$ in BA were prepared in methanol. Appropriate volumes of additional methanol were added as needed to dilute to desired concentrations, with total volumes made up to $50.0 \mathrm{~mL}$. Round-bottomed flasks containing solutions were left open to allow exposure to oxygen and stirred for 48-72 hours. Measurement of UV-visible absorbances were carried out by decanting solutions into a graduated cylinder at time of measurement, making up the total volume to $50.0 \mathrm{~mL}$ with methanol to compensate for evaporation, decanting back into the round-bottom flask and stirring for 5 minutes to achieve homogeneity. Samples were then transferred into a quartz cuvette for analysis. Single-neck (1" diameter) round-bottom flasks were chosen as reaction vessels after verifying that rates of 
evaporation of stirred methanol at room temperature ensured that solution volumes never fell below $40 \mathrm{~mL}$ during the course of the reaction.

Preparation of genipin-embedded calcium alginate gel beads. 1, 10, 50, or $100 \mathrm{mg}$ of genipin was dissolved in $10.0 \mathrm{~mL}$ of distilled water in a $100 \mathrm{~mL}$ beaker. In the case of the addition of 100 $\mathrm{mg}$ of genipin, heating to $80{ }^{\circ} \mathrm{C}$ was required for full dissolution. $200 \mathrm{mg}$ of sodium alginate powder was added with vigorous stirring to form the sodium alginate gel. Using a variation of the simple dripping (extrusion) technique described by Bhandari et. al., ${ }^{21}$ a $1 \mathrm{~mL}$ disposable syringe without a needle was employed to uptake $1.00 \mathrm{~mL}$ of sodium alginate gel. Due to dripping of gel from the outside of the syringe, the first 1-2 drops were dispensed back into the gel mixture. The remainder was dispensed dropwise from a height of $10 \mathrm{~cm}$ above the surface of $25.0 \mathrm{~mL}$ of a $1.0 \% \mathrm{w} / \mathrm{v} \mathrm{CaCl}_{2}$ solution contained in a $125 \mathrm{~mL}$ Erlenmeyer flask. 12-15 beads were dispensed in this manner until the syringe contained $>0.1 \mathrm{~mL}$ of gel, at which point the syringe was re-filled to avoid size decrease of gel beads due to air bubbles inside the syringe, and lack of material. After $100-110$ beads had been dispensed in this manner, using $75-80 \%$ of the sodium alginate gel mass, the remaining $\mathrm{CaCl}_{2}$ solution was decanted from the beads and the beads were washed with $10 \mathrm{~mL}$ of an aqueous solution of $0.1 \mathrm{M} \mathrm{CH} \mathrm{COOH}_{3} \mathrm{CH}{ }_{3} \mathrm{COONa} \mathrm{pH} 4$ buffer. Beads were then dispensed onto a watch glass, separated using tweezers and permitted to stand 1 hour to allow excess water to run onto the glass. The beads were then removed from the watch glass and stored in a sealed vial in a refrigerator at $4{ }^{\circ} \mathrm{C}$.

General Procedure for reaction of genipin immobilized in calcium alginate gel beads with primary amine vapors. 1-10 genipin-embedded calcium alginate gel beads were placed in a 20 $\mathrm{mL}$ vial using a clean pair of tweezers. $0.10 \mathrm{mmol}$ of primary amine was added to a 1-dram vial (or Eppendorf tube) which was placed, uncapped, inside the 4-dram vial containing the beads, and the larger vial was sealed with a cap and either left at room temperature or placed in a refrigerator at $4{ }^{\circ} \mathrm{C}$ and monitored visually for up to 48 hours days.

In the case where ten gel beads were exposed to $0.10 \mathrm{mmol}$ amine, after 24 hours at room temperature the gel beads remained colourless, while after 48 hours all ten beads had turned blue. In the case where only one bead was used instead of ten beads, the single bead turned deep blue within 24 hours.

To achieve dissolution of gel beads to obtain UV-vis spectra after specified reaction times of 648 hours, 10 dyed genipin-containing gel beads were added to $3.5 \mathrm{~mL}$ of a $55 \mathrm{mM}$ solution of sodium citrate to allow dissolution of gel beads by cation exchange mediated by the citrate cation chelator. ${ }^{22}$ Although some of the blue dye became insoluble particulate in this aqueous solution, precluding qualitative UV-vis absorption measurements, enough remained in solution to qualitatively observe the blue dye by UV-vis spectroscopy.

Reaction of genipin immobilized in calcium alginate gel beads with raw chicken. 10 genipinembedded calcium alginate gel beads produced no more than seven days previously were placed in each of two $20 \mathrm{~mL}$ vials using a clean pair of tweezers. A piece of chicken breast 
purchased within the previous 24 hours from a commercial grocery store was cut into slices of mass 5-6 grams and one slice was placed in each vial using a separate, clean pair of tweezers, such that the gel beads were separated from the surface of the raw chicken. The vials were sealed with a plastic screw cap. One was left at room temperature, while one was refrigerated at $4^{\circ} \mathrm{C}$. Both samples were monitored visually and photographed at hours 1, 2, 3 and 6 .

\section{Results and Discussion}

To confirm the selectivity of genipin for primary amines, the reactions with a series of amines in methanol solutions were monitored over periods of 48-72 hours at room temperature. These reactions were performed at concentrations of $2.0 \times 10^{-3} \mathrm{M}$ for both genipin and amine, in open vessels to permit diffusion of $\mathrm{O}_{2}$ into the system to promote blue dye formation. Benzylamine, $\mathrm{N}$-methylbenzylamine, and $\mathrm{N}, \mathrm{N}$-dimethylbenzylamine were initially tested to represent a series of simple, analogous primary, secondary and tertiary amines, and reactions were monitored by UV-vis absorbance spectroscopy. Consistent with reported literature, formation of blue dye was only observed in the reaction of genipin with the primary benzylamine, with strong visible absorbance exhibited between 550-650 nm (Figure S4). Though solutions of tertiary and secondary amines, $\mathrm{N}, \mathrm{N}$-dimethylbenzylamine and $\mathrm{N}$-methylbenzylamine, did exhibit a colour change to reddish golden, they did not form the blue dye even after extended reaction times. Monitoring the reactions of $\mathrm{N}, \mathrm{N}$-dimethylbenzylamine and $\mathrm{N}$-methylbenzylamine, by ${ }^{1} \mathrm{H} \mathrm{NMR}$ spectroscopy with genipin at greater concentrations does indicate a reaction between genipin and amine but it produced an intractable mixture of products. These studies unequivocally show the selectivity of genipin to react with primary amines to form a blue product.

Reactions with biogenic amines putrescine, cadaverine, tyramine and histamine also exhibited formation of deep blue dye (Figure 1). In these cases, an incubation period of 12-24 hours was required before strong absorbances with maxima at 590-610 nm were observed. The incubation time is attributable to the time required for growth of conjugated oligomers absorbing in the red region. Although reactions were monitored for up to 120 hours, in all cases, $\lambda_{\max }$ and molar attenuation coefficient values demonstrated only minimal change after 48 hours (e.g. Figure S5). Interestingly, molar attenuation coefficients varied widely between reactions of genipin with different $\mathrm{BA}$, ranging from $1.2 \times 10^{2} \mathrm{Lmol}^{-1} \mathrm{~cm}^{-1}$ in putrescine to $4.4 \times 10^{3} \mathrm{Lmol}^{-1} \mathrm{~cm}^{-1}$ in tyramine. It is not obvious from differences in molecular structure of the amines why significant variance is observed in molar attenuation coefficients of the solutions of dyes. 


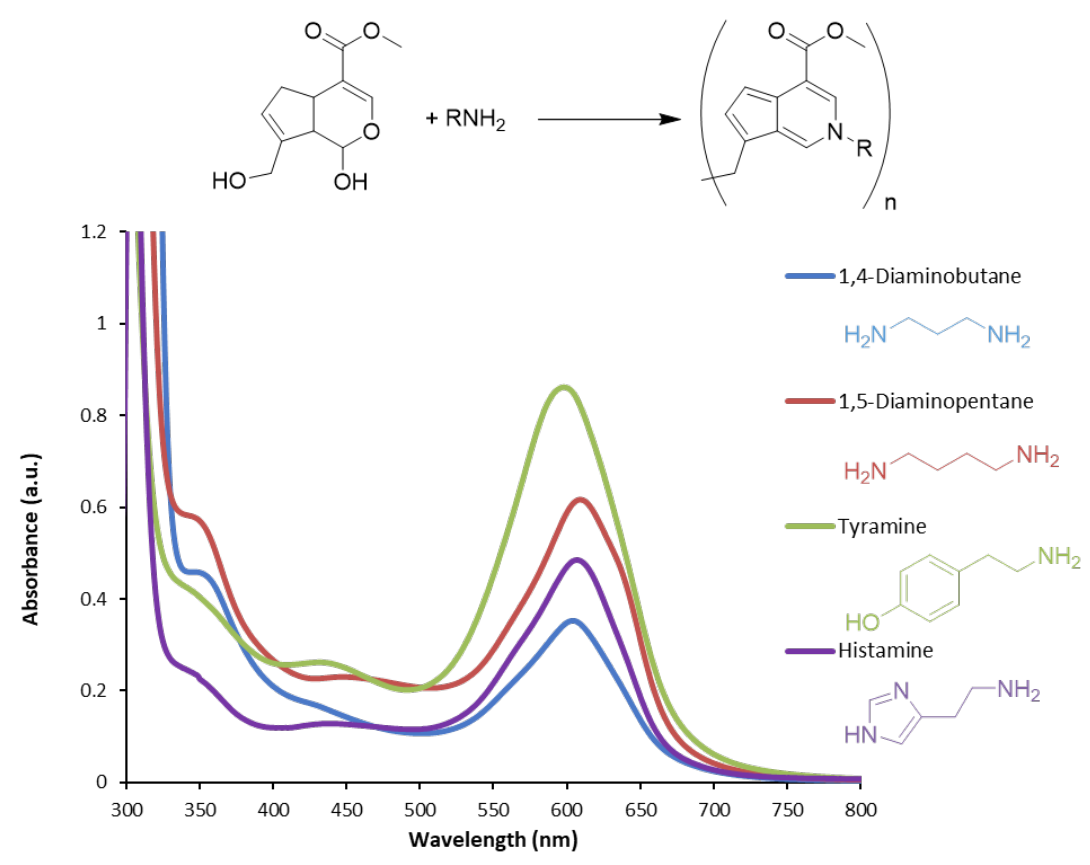

Figure 1 - Stacked UV-vis Spectra for the reaction of genipin with BAs. Dilution factors - 1,4diaminobutane (no dilution); 1,5-diaminopentane (5x dilution); Tyramine (10x dilution); Histamine (20x dilution).

Unsurprisingly, when reactions were carried out at increased concentrations of genipin and amine, the blue dye formed much more rapidly, for example within 4 hours at concentrations of $0.20 \mathrm{M}$ (Figure S10)).

From these solution studies, the limit of visual detection of amines with a $0.50 \mathrm{M}$ solution of genipin in 50:50 methanol:water was found to vary depending on BA used. Putrescine and tyramine, the tested amines whose dyes exhibited the lowest and highest molar attenuation coefficients, respectively, were examined. Solutions of genipin in methanol exhibit a dull golden tinge, while presence of the blue dyes were confirmed visually by a change in colour to dull green, the result of the presence of both blue dye and the golden hue from remaining unreacted genipin. Tyramine could be visually detected at concentrations of $6.9 \mathrm{ppm}$ after 24 hours (Figure S27), while the detection limit for putrescine was nearly an order of magnitude higher at $44 \mathrm{ppm}$ after 24 hours. This trend correlates with the measured trend in molar attenuation coefficient measured for the dyes incorporating putrescine or tyramine units (converted from molarity).

Enticingly, the reaction of putrescine with genipin in the absence of oxygen produced a different response (Figure 2). Using degassed water and an atmosphere of nitrogen, the solutions produced a golden red coloration with no indication of the blue response as indicated by the $\lambda_{\max }$ at $\sim 600 \mathrm{~nm}$. Upon exposure to air after 48 hours, the solutions rapidly turned blue. To explore the sensitivity of the genipin and amine intermediate to oxygen gas, we found even $0.5 \%$ (5000 ppm) of oxygen gas (converted from moles of $\mathrm{H}_{2} \mathrm{O}_{2}$, see $\mathrm{SI}$ ) added under an 
atmosphere of $\mathrm{N}_{2}$ were enough to trigger the oxidative oligomerization reaction turning the sample blue over a period of 24 hours. This sensitivity is below the oxygen threshold established in modified atmospheric packaging $(\sim 2 \%)$ and in the desired range of sensitivity $\left(0.5-2 \% \mathrm{O}_{2}\right.$ level). ${ }^{16}$

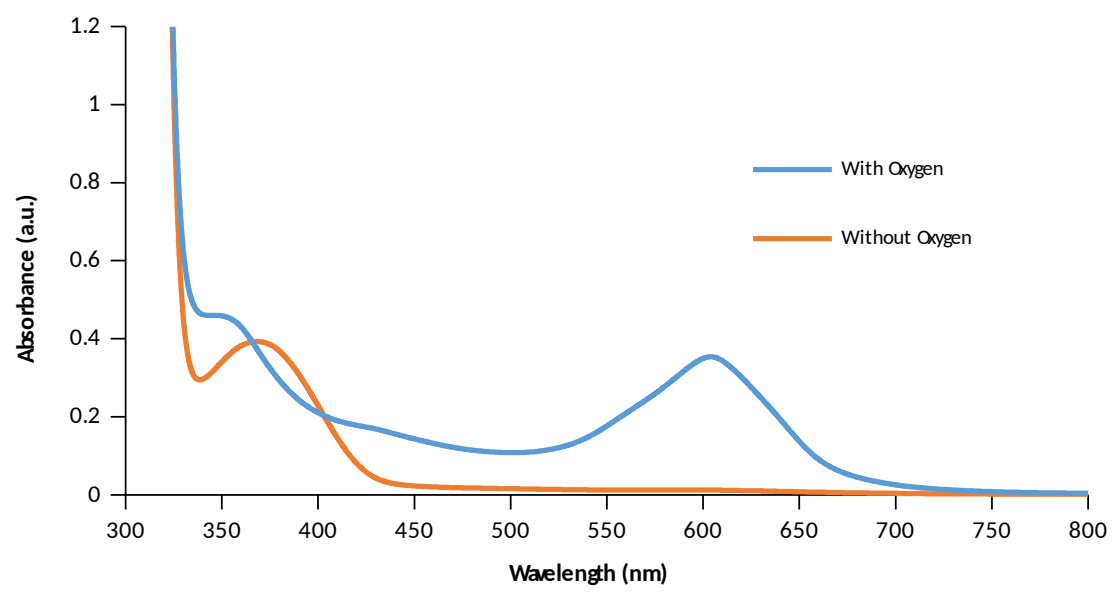

Figure 2 - UV-vis absorbance spectra of $2.0 \mathrm{mM}$ solutions of genipin in methanol in reaction with $2.0 \mathrm{mM}$ of 1,4-diaminobutane in the presence (blue) and absence (red) of $\mathrm{O}_{2}$ after 48 hours.

To demonstrate the applicability of our sensing platform for food spoilage detection, an immobilization method for genipin was developed using a calcium alginate gel matrix. Alginate gels are common edible food thickening agents derived from alginic acid sourced from seaweed or brown algae. ${ }^{22}$ Calcium alginate gel beads are easily produced from sodium alginate and permit penetration of small molecules through their pores. ${ }^{21}$ Genipin was embedded in these beads through modifying a literature procedure for the impregnation of calcium alginate gel beads with enzymes. ${ }^{21}$ After extensive screening, it was found that a concentration of $5 \mathrm{mg} / \mathrm{mL}$ of genipin in water, approximately equivalent to the room-temperature solubility of genipin in water, resulted in the most responsive beads. As a proof of concept, we selected the most volatile biogenic amine, putrescine, to test the sensing capabilities of the beads. Immobilized genipin-containing beads were exposed to the gaseous BA by adding $0.1 \mathrm{mmol}$ of putrescine to an open 1-dram vial and sealing the 1-dram vial inside a 4-dram vial containing 10 genipininfused beads. The beads responded as expected and a blue colour developed upon exposure to BA vapors within 24 hours (Figure 3). To mimic conditions for food storage, these experiments were also undertaken at $4{ }^{\circ} \mathrm{C}$, and encouragingly the beads did not exhibit any color change, even after 48 hours (Figure S28). Gel beads could subsequently be dissolved in an aqueous citric acid solution for UV-vis absorbance analysis (Figure 3), although undissolved particulate matter interfered with quantitative measurements.

In analyzing the absorbance of solutions obtained from dissolving gel beads exposed for 48 hours to vials containing varying amounts of putrescine (Figure S29), it is evident that increasing 
amounts of amine result in increased intensity of the blue dye. The vapor pressure of linear putrescine has been reported as $259.3 \mathrm{~Pa}$ at $300.7 \mathrm{~K} .{ }^{23}$ Using the reported vapor pressure at $300.7 \mathrm{~K}$ as an approximation for the vapor pressure at room temperature, it can be calculated from the ideal gas law that for the $18 \mathrm{~mL}$ closed volume of the 4-dram vial, evaporation of $<0.2$ $\mathrm{mg}$ of putrescine is required to reach equilibrium vapor pressure. Upon dissolution after reaction, solutions of gel beads exposed to $0.3 \mathrm{mg}, 1.2 \mathrm{mg}$ and $12 \mathrm{mg}$ of putrescine in open onedram vials demonstrated significantly increased absorbance as the amount of amine was increased (Figure S29). Thus, it can be hypothesized that the intensity of the absorbance is more correctly correlated to the absolute amount of amine available for reaction, and the identity of that amine, than the vapor pressure.
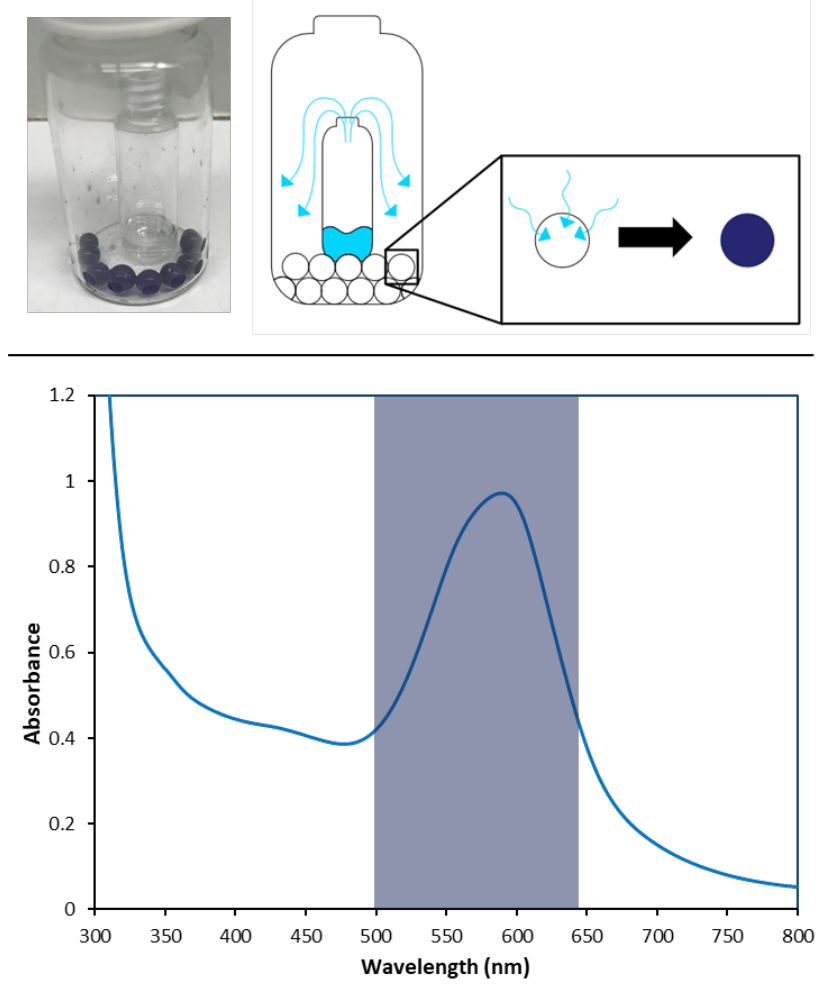

Figure 3: Genipin-impregnated beads after 24 hours exposure to putrescine vapours at room temperature

Having established the sensor's response to gaseous amines, we sought to test them against real food samples. Samples of chicken breast (5-6 grams each) were freshly prepared and stored in sealed vials with the genipin-impregnated beads. With the beads in close proximity to the meat, the sensors behaved as expected and turned blue upon exposure. Samples were stored both at room temperature and in a refrigerator at $4{ }^{\circ} \mathrm{C}$ to mimic typical storage conditions. To quantify the color difference between the beads, $\Delta E^{*}$ values were determined following commission internationale de l'éclairage (CIE) guidelines (Figure 4). $A \Delta E^{*}$ value $>5$ is considered to be distinguishable by the naked eye. ${ }^{12}$ This approach has previously been used to determine 
the effectiveness of small molecule amine sensors. ${ }^{12}$ The sensors were monitored over the course of 6 hours with images taken at time points of 1, 2, 3 and 6 hours (Table S1). $\Delta E^{*}$ values of 38 and 0 were obtained after 6 hours for the samples at room temperature and at $4{ }^{\circ} \mathrm{C}$, respectively. These values correspond to a significant blue coloration of the beads at room temperature versus no visible change in the refrigerator (Figure 4). The rate of colour appearance in these indicator beads is consistent with the USDA health advisory on raw poultry, which warns against leaving raw poultry at room temperature for longer than two hours. ${ }^{24}$

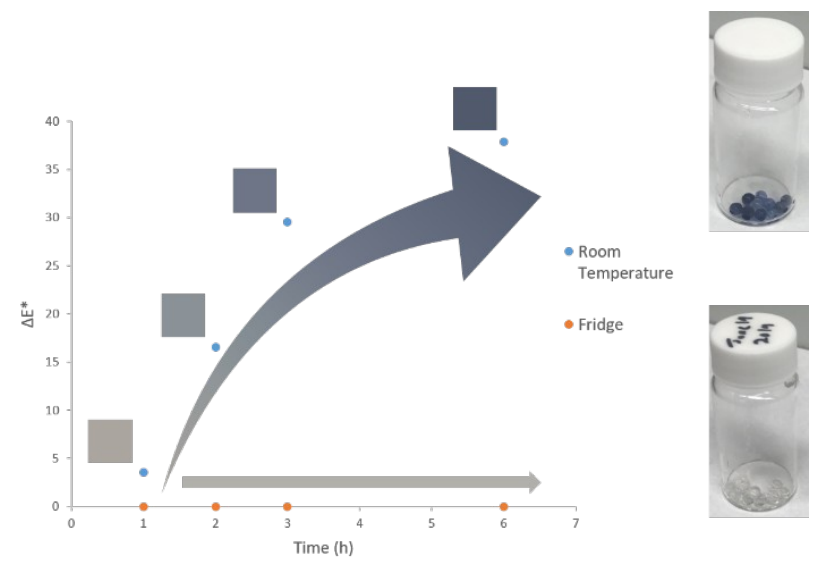

Figure 4: Change in color of Genipin Impregnated Beads in the presence of chicken, room temperature (blue) and $4{ }^{\circ} \mathrm{C}$ (orange).

\section{Conclusion}

In summary, this proof-of-principal study indicates that genipin can act as a highly selective dual sensor for both biogenic amines and $\mathrm{O}_{2}$. This uniquely positions genipin as an ideal colorimetric sensor for food spoilage as it can detect the root cause of this process through off-gases. Moreover, by immobilizing the sensor in an edible calcium alginate matrix, biogenic amine vapors were detected at very low concentrations. This was extended to monitoring chicken spoilage by the naked eye, indicating its potential in food spoilage detection.

\section{Acknowledgement}

The authors gratefully acknowledge financial support from NSERC of Canada, the Canadian Foundation for Innovation and Inkbox Ink. CBC is grateful for the award of a Canada Research Chair. The authors thank Professor Thomas Baumgartner for the use of the UV-vis spectrophotometer and valuable feedback.

\section{References}

1. Santos MHS. Biogenic amines: their importance in foods. Int J Food Microbiol. 29:213-31 (1996);

2. Önal A. A review: Current analytical methods for the determination of biogenic amines in foods. Food Chem. 103:1475-86 (2007); 
3. Naila A, Flint S, Fletcher G, Bremer P, Meerdink G. Control of Biogenic Amines in FoodExisting and Emerging Approaches. J Food Sci. 75:R139-50 (2010);

4. $\quad$ Liu SF, Petty AR, Sazama GT, Swager TM. Single-Walled Carbon Nanotube/Metalloporphyrin Composites for the Chemiresistive Detection of Amines and Meat Spoilage. Angew. Chem. Int Ed. 54:6554-7 (2015);

5. Aschemann-Witzel J. Waste not, want not, emit less: Reducing food waste in the supply chain and at home can help to reduce carbon emissions. Vol. 352, Science. 352:408-9 (2016);

6. Sotzing GA, Phend JN, Grubbs RH, Lewis NS. Highly Sensitive Detection and Discrimination of Biogenic Amines Utilizing Arrays of Polyaniline/Carbon Black Composite Vapor Detectors. Chem Mater. 12:593-5 (2000);

7. Yousefi H, Ali MM, Su H-M, Filipe CDM, Didar TF. Sentinel Wraps: Real-Time Monitoring of Food Contamination by Printing DNAzyme Probes on Food Packaging. ACS Nano. 12:3287-94 (2018);

8. Barandun G, Soprani M, Naficy S, Grell M, Kasimatis M, Chiu KL, Ponzoni A, Güder F. Cellulose Fibers Enable Near-Zero-Cost Electrical Sensing of Water-Soluble Gases. ACS Sensors. 4:1662-9 (2019);

9. Chopra S, Singh A, Venugopalan P, Singh N, Kaur N. Organic nanoparticles for visual detection of spermidine and spermine in vapors and aqueous phase. ACS Sustain Chem Eng. 5:1287-96 (2017)

10. Valderrey V, Bonasera A, Fredrich S, Hecht S. Light-Activated Sensitive Probes for Amine Detection. Angew. Chem. Int Ed. 56:1914-8 (2017);

11. Li L, Li W, Ran X, Wang L, Tang H, Cao D. A highly efficient, colorimetric and fluorescent probe for recognition of aliphatic primary amines based on a unique cascade chromophore reaction. Chem Commun. 55:9789-92 (2019);

12. Diaz YJ, Page ZA, Knight AS, Treat NJ, Hemmer JR, Hawker CJ, Read De Alaniz J. A Versatile and Highly Selective Colorimetric Sensor for the Detection of Amines. Chem. Eur J. 23:3562-6 (2017);

13. Chen Q, Diaz YJ, Hawker MC, Martinez MR, Page ZA, Xiao-An Zhang S, Hawker CJ, Read de Alaniz J. Stable Activated Furan and Donor-Acceptor Stenhouse Adduct Polymer Conjugates as Chemical and Thermal Sensors. Macromolecules. 52:4370-5 (2019);

14. Dudnyk I, Janeček E-R, Vaucher-Joset J, Stellacci F. Edible sensors for meat and seafood freshness. Sensors Actuators B Chem. 259:1108-12 (2018);

15. Huang X, Zou X, Shi J, Guo Y, Zhao J, Zhang J, Hao L. Determination of pork spoilage by colorimetric gas sensor array based on natural pigments. Food Chem. 145:549-54 (2014);

16. Mills A. Oxygen indicators and intelligent inks for packaging food. Chem. Soc. Rev. 34:1003-11 (2005). 
17. Yan LP, Wang YJ, Ren L, Wu G, Caridade SG, Fan JB, Wang LY, Ji PH, Oliveira JM, Oliveira JT, Mano JF, Reis RL. Genipin-cross-linked collagen/chitosan biomimetic scaffolds for articular cartilage tissue engineering applications. J Biomed Mater Res Part A. 95A:465-75 (2010);

18. Butler MF, Ng Y-F, Pudney PDA. Mechanism and kinetics of the crosslinking reaction between biopolymers containing primary amine groups and genipin. J Polym Sci Part A Polym Chem. 41:3941-53 (2003);

19. Tsai C-C, Huang R-N, Sung H-W, Liang HC. In vitro evaluation of the genotoxicity of a naturally occurring crosslinking agent (genipin) for biologic tissue fixation. J Biomed Mater Res. 52:58-65 (2000);

20. Touyama R, Takeda Y, Inoue K, Kawamura Ik, Yatsuzuka M, Ikumoto T, Shingu T, Yokoi T, Inouye H. Studies on the Blue Pigments Produced from Genipin and Methylamine. I. Structures of the Brownish-Red Pigments, Intermediates Leading to the Blue Pigments. Chem Pharm Bull. 42:668-73 (1994);

21. Ching SH, Bansal N, Bhandari B. Alginate gel particles-A review of production techniques and physical properties. Crit Rev Food Sci Nutr. 57:1133-52 (2017);

22. Goh CH, Heng PWS, Chan LW. Alginates as a useful natural polymer for microencapsulation and therapeutic applications. Vol. 88, Carbohydrate Polymers. p. 1122012.

23. Pozdeev VA, Verevkin SP. Vapor pressure and enthalpy of vaporization of linear aliphatic alkanediamines. J Chem Thermodyn. 43:1791-9 (2011);

24. https://www.fsis.usda.gov/wps/portal/fsis/topics/food-safety-education/getanswers/food-safety-fact-sheets/safe-food-handling/keep-food-safe-food-safetybasics/ct_index 
